\title{
Search for squarks and gluinos with the ATLAS detector in final states with jets and missing transverse momentum using $4.7 \mathrm{fb}^{-1}$ of $\sqrt{s}=7 \mathrm{TeV}$ proton-proton collision data
}

\author{
G. Aad et al.* \\ (ATLAS Collaboration) \\ (Received 4 August 2012; published 22 January 2013)
}

\begin{abstract}
A search for squarks and gluinos in final states containing jets, missing transverse momentum and no high- $p_{\mathrm{T}}$ electrons or muons is presented. The data represent the complete sample recorded in 2011 by the ATLAS experiment in $7 \mathrm{TeV}$ proton-proton collisions at the Large Hadron Collider, with a total integrated luminosity of $4.7 \mathrm{fb}^{-1}$. No excess above the Standard Model background expectation is observed. Gluino masses below $860 \mathrm{GeV}$ and squark masses below $1320 \mathrm{GeV}$ are excluded at the $95 \%$ confidence level in simplified models containing only squarks of the first two generations, a gluino octet and a massless neutralino, for squark or gluino masses below $2 \mathrm{TeV}$, respectively. Squarks and gluinos with equal masses below $1410 \mathrm{GeV}$ are excluded. In minimal supergravity/constrained minimal supersymmetric Standard Model models with $\tan \beta=10, A_{0}=0$ and $\mu>0$, squarks and gluinos of equal mass are excluded for masses below $1360 \mathrm{GeV}$. Constraints are also placed on the parameter space of supersymmetric models with compressed spectra. These limits considerably extend the region of supersymmetric parameter space excluded by previous measurements with the ATLAS detector.
\end{abstract}

DOI: 10.1103/PhysRevD.87.012008

PACS numbers: 12.60.Jv, 13.85.Rm, 14.80.Ly

\section{INTRODUCTION}

Many extensions of the Standard Model (SM) include heavy colored particles, some of which could be accessible at the Large Hadron Collider (LHC) [1]. The squarks and gluinos of supersymmetric (SUSY) theories [2-10] form one class of such particles. This paper presents a new ATLAS search for squarks and gluinos in final states containing only jets and large missing transverse momentum. Interest in this final state is motivated by the large number of $R$-parity conserving models, including minimal supergravity (MSUGRA)/constrained minimal supersymmetric Standard Model (CMSSM) scenarios [11-15], in which squarks $\tilde{q}$ and gluinos $\tilde{g}$ can be produced in pairs $(\{\tilde{g} \tilde{g}, \tilde{q} \tilde{q}, \tilde{q} \tilde{g}\})$ and can generate the final state of interest through their direct $\left(\tilde{q} \rightarrow q \tilde{\chi}_{1}^{0}\right.$ and $\left.\tilde{g} \rightarrow q \bar{q} \tilde{\chi}_{1}^{0}\right)$ and cascade decays to weakly interacting neutralinos $\tilde{\chi}_{1}^{0}$, which escape the detector unseen. "Squark" here refers only to the superpartners of the four light-flavor quarks. The analysis presented here is based on a study of final states which are reconstructed as purely hadronic. Events with reconstructed electrons or muons are vetoed to avoid overlap with a related ATLAS search [16] that requires them. The term "leptons" is therefore used in this paper to refer only to reconstructed electrons and muons and does not include $\tau$ leptons. Compared to previous studies [17], this updated analysis uses the full data set $\left(4.7 \mathrm{fb}^{-1}\right)$ recorded at $\sqrt{s}=7 \mathrm{TeV}$ in

*Full author list given at the end of the article.

Published by the American Physical Society under the terms of the Creative Commons Attribution 3.0 License. Further distribution of this work must maintain attribution to the author(s) and the published article's title, journal citation, and DOI.
2011 and extends the sensitivity of the search by selecting final state topologies with higher jet multiplicities. The search strategy is optimized for maximum discovery reach in the $\left(m_{\tilde{g}}, m_{\tilde{q}}\right)$ plane (where $m_{\tilde{g}}, m_{\tilde{q}}$ are the gluino and squark masses, respectively) for a range of models. This includes a simplified model in which all other supersymmetric particles, except for the lightest neutralino, are given masses beyond the reach of the LHC. Although interpreted in terms of SUSY models, the main results of this analysis (the data and expected background event counts in the signal regions) are relevant for constraining any model of new physics that predicts the production of jets in association with missing transverse momentum.

The paper begins with a brief description of the ATLAS detector (Sec. II), followed by an overview of the analysis strategy (Sec. III). This is followed by short descriptions of the data and Monte Carlo (MC) simulation samples used (Sec. IV) and of the trigger strategy (Sec. V). Section VI describes the physics object definitions. Section VII describes the event cleaning techniques used to reject noncollision backgrounds, while Sec. VIII describes the final event selections and resulting event counts. Section IX describes the techniques used to estimate the SM backgrounds, with the systematic uncertainties summarized in Sec. X. Section XI describes the statistical model used to interpret the observations and presents the results in terms of constraints on SUSY model parameter space. Finally Sec. XII summarizes the main results and conclusions.

\section{THE ATLAS DETECTOR}

The ATLAS detector [18] is a multipurpose particle physics apparatus with a forward-backward symmetric cylindrical geometry and nearly $4 \pi$ coverage in solid 
angle [19]. The layout of the detector features four superconducting magnet systems, which comprise a thin solenoid surrounding inner tracking detectors and three large toroids used in a large muon spectrometer. Located between these two detector systems, the calorimeters are of particular importance to this analysis. In the pseudorapidity region $|\eta|<3.2$, high-granularity liquid-argon (LAr) electromagnetic (EM) sampling calorimeters are used. An iron/scintillator-tile calorimeter provides hadronic coverage over $|\eta|<1.7$. The end-cap and forward regions, $1.5<|\eta|<4.9$, are instrumented with LAr calorimeters for both EM and hadronic measurements.

\section{ANALYSIS STRATEGY}

This analysis aims to search for the production of heavy SUSY particles decaying into jets and neutralinos, with the latter creating missing transverse momentum $\left(E_{\mathrm{T}}^{\mathrm{miss}}\right)$. Because of the high mass scale expected for the SUSY signal, the "effective mass" $m_{\text {eff }}$ (defined below) is a powerful discriminant between the signal and most SM backgrounds. The requirements used to select jets and leptons (which are referred to as physics objects) are chosen to give sensitivity to a broad range of SUSY models. In order to achieve maximal reach over the $\left(m_{\tilde{g}}, m_{\tilde{q}}\right)$ plane, six analysis channels are defined. Squarks typically generate at least one jet in their decays, for instance through $\tilde{q} \rightarrow q \tilde{\chi}_{1}^{0}$, while gluinos typically generate at least two jets, for instance through $\tilde{g} \rightarrow q \bar{q} \tilde{\chi}_{1}^{0}$. Processes contributing to $\tilde{q} \tilde{q}, \tilde{q} \tilde{g}$ and $\tilde{g} \tilde{g}$ final states therefore lead to events containing at least two, three or four jets, respectively. Cascade decays of heavy particles, as well as initial and final state radiation, tend to further increase the final state multiplicity.

Inclusive analysis channels, labeled A-E and characterized by increasing minimum jet multiplicity from two to six, are therefore defined. In addition, the two-jet sample is divided into two channels, $\mathrm{A}$ and $\mathrm{A}^{\prime}$, using the ratio of $E_{\mathrm{T}}^{\mathrm{miss}}$ to $m_{\text {eff }}$, giving a total of six channels. Channel $\mathrm{A}^{\prime}$ is designed to improve the sensitivity to models with small supersymmetric particle ("sparticle") mass splittings, where the presence of initial state radiation jets may allow signal events to be selected irrespective of the visibility of the sparticle decay products. The lower jet multiplicity channels focus on models characterized by squark pair production with short decay chains, while those requiring high jet multiplicity are optimized for gluino pair production and/or long cascade decay chains. The final limits are set using the channel with the best expected sensitivity for each hypothesis. The channels and signal regions (SRs) are summarized in Table I. The final selection criteria are defined without reference to collision data satisfying the criteria applied earlier in the selection.

The effective mass is defined to be the scalar sum of the transverse momenta of the leading $N$ jets in the event together with $E_{\mathrm{T}}^{\text {miss }}$ :

$$
m_{\mathrm{eff}} \equiv \sum_{i=1}^{N} p_{\mathrm{T}}^{(i)}+E_{\mathrm{T}}^{\mathrm{miss}} .
$$

This general quantity is used to select events in two different ways, for which the specific values of $N$ used in the sum differ. Criteria are placed on the ratio of $E_{\mathrm{T}}^{\text {miss }}$ to $m_{\text {eff }}$, in which context $N$ is defined to be the minimum number of jets used in the channel under consideration (for example $N=2$ for channel A). In Table I, where the number of jets used is explicitly notated, the expression $m_{\text {eff }}(\mathrm{Nj})$ indicates the exact, exclusive, number of jets used. However, the final signal selection in all channels uses criteria on a more inclusive definition, $m_{\mathrm{eff}}$ (incl.), for which the sum extends over all jets with $p_{\mathrm{T}}>40 \mathrm{GeV}$.

TABLE I. Criteria used to define each of the inclusive channels and streams in the analysis. The jets are ordered with the highest $p_{\mathrm{T}}$ first. The variables used are defined in the text. The $E_{\mathrm{T}}^{\mathrm{miss}} / m_{\mathrm{eff}}$ selection in any $N$ jet channel uses a value of $m_{\mathrm{eff}}$ constructed from only the leading $N$ jets (indicated in parentheses). However, the final $m_{\text {eff }}$ (incl.) selection, which is used to define the signal regions, includes all jets with $p_{\mathrm{T}}>40 \mathrm{GeV}$. The three $m_{\mathrm{eff}}$ (incl.) values listed in the final row denote the tight, medium and loose selections, respectively, as used for the final SRs.

\begin{tabular}{|c|c|c|c|c|c|c|}
\hline \multirow[b]{2}{*}{ Requirement } & \multicolumn{6}{|c|}{ Channel } \\
\hline & A & $\mathrm{A}^{\prime}$ & $\mathrm{B}$ & $\mathrm{C}$ & $\mathrm{D}$ & $\mathrm{E}$ \\
\hline Trigger & \multicolumn{6}{|c|}{ Leading jet $p_{\mathrm{T}}>75 \mathrm{GeV}$ (EM scale) and $E_{\mathrm{T}}^{\text {miss }}>45-55 \mathrm{GeV}$} \\
\hline Lepton veto & \multicolumn{6}{|c|}{ No electron (muon) with $p_{\mathrm{T}}>20(10) \mathrm{GeV}$} \\
\hline$E_{\mathrm{T}}^{\text {miss }}[\mathrm{GeV}]>$ & \multicolumn{6}{|c|}{160} \\
\hline$p_{\mathrm{T}}\left(j_{1}\right)[\mathrm{GeV}]>$ & \multicolumn{6}{|c|}{130} \\
\hline$p_{\mathrm{T}}\left(j_{2}\right)[\mathrm{GeV}]>$ & \multicolumn{6}{|c|}{60} \\
\hline$p_{\mathrm{T}}\left(j_{3}\right)[\mathrm{GeV}]>$ & $\cdots$ & $\cdots$ & 60 & 60 & 60 & 60 \\
\hline$p_{\mathrm{T}}\left(j_{4}\right)[\mathrm{GeV}]>$ & $\cdots$ & $\cdots$ & $\cdots$ & 60 & 60 & 60 \\
\hline$p_{\mathrm{T}}\left(j_{5}\right)[\mathrm{GeV}]>$ & $\cdots$ & $\cdots$ & $\cdots$ & $\cdots$ & 40 & 40 \\
\hline$p_{\mathrm{T}}\left(j_{6}\right)[\mathrm{GeV}]>$ & $\cdots$ & $\cdots$ & $\cdots$ & $\ldots$ & $\ldots$ & 40 \\
\hline$\Delta \phi\left(\text { jet }_{i}, \vec{P}_{\mathrm{T}}^{\text {miss }}\right)_{\min }[\mathrm{rad}]>$ & \multicolumn{3}{|c|}{$0.4(i=\{1,2,(3)\})$} & \multicolumn{3}{|c|}{$0.4(i=\{1,2,3\}), 0.2\left(p_{\mathrm{T}}>40 \mathrm{GeV}\right.$ jets $)$} \\
\hline$E_{\mathrm{T}}^{\text {miss }} / m_{\mathrm{eff}}(N \mathrm{j})>$ & $0.3(2 j)$ & $0.4(2 j)$ & $0.25(3 j)$ & $0.25(4 j)$ & $0.2(5 j)$ & $0.15(6 j)$ \\
\hline$m_{\mathrm{eff}}($ incl. $)[\mathrm{GeV}]>$ & $1900 / 1400 / \cdots$ & $\cdots / 1200 / \cdots$ & $1900 / \cdots / \cdots$ & $1500 / 1200 / 900$ & $1500 / \cdots / \cdots$ & $1400 / 1200 / 900$ \\
\hline
\end{tabular}


Requirements on $m_{\text {eff }}$ and $E_{\mathrm{T}}^{\text {miss }}$, which suppress the QCD multijet background, formed the basis of the previous ATLAS jets $+E_{\mathrm{T}}^{\text {miss }}+0$-lepton SUSY search [17]. The same strategy is adopted in this analysis.

In Table I, $\Delta \phi\left(\text { jet }_{i}, \vec{P}_{\mathrm{T}}{ }^{\text {miss }}\right)_{\min }$ is the smallest of the azimuthal separations between the missing momentum vector in the transverse plane, $\vec{P}_{\mathrm{T}}{ }^{\text {miss }}$, and the reconstructed jets. For channels $\mathrm{A}, \mathrm{A}^{\prime}$ and $\mathrm{B}$, the selection requires $\Delta \phi\left(\text { jet }_{i}, \vec{P}_{\mathrm{T}}{ }^{\text {miss }}\right)_{\min }>0.4 \mathrm{rad}$ using up to three leading jets. For the other channels an additional requirement $\Delta \phi\left(\text { jet }_{i}, \vec{P}_{\mathrm{T}}{ }^{\text {miss }}\right)_{\min }>0.2 \mathrm{rad}$ is applied to the remaining jets with $p_{\mathrm{T}}>40 \mathrm{GeV}$. Requirements on $\Delta \phi\left(\right.$ jet $_{i}$, $\left.\vec{P}_{\mathrm{T}}^{\text {miss }}\right)_{\min }$ and $E_{\mathrm{T}}^{\text {miss }} / m_{\text {eff }}$ are designed to reduce the background from multijet processes.

SM background processes contribute to the event counts in the signal regions. The dominant sources are: $W+$ jets, $Z+$ jets, top quark pair, single top quark, diboson and multijet production. The majority of the $W+$ jets background is composed of $W \rightarrow \tau \nu$ events, or $W \rightarrow e \nu, \mu \nu$ events in which no electron or muon candidate is reconstructed. The largest part of the $Z+$ jets background comes from the irreducible component in which $Z \rightarrow \nu \bar{\nu}$ decays generate large $E_{\mathrm{T}}^{\text {miss }}$. Top quark pair production followed by semileptonic decays, in particular $t \bar{t} \rightarrow b \bar{b} q q^{\prime} \tau \nu$ with the $\tau$ lepton decaying hadronically, as well as single top quark events, can also generate large $E_{\mathrm{T}}^{\mathrm{miss}}$ and pass the jet and lepton requirements at a non-negligible rate. The multijet background in the signal regions is caused by poor reconstruction of jet energies in the calorimeters leading to apparent missing transverse momentum, as well as by neutrino production in semileptonic decays of heavy quarks. Extensive validation of the MC simulation against data has been performed for each of these background sources and for a wide variety of control regions (CRs).

Each of the six channels is used to construct between one and three signal regions with "tight," "medium" and/ or "loose" $m_{\text {eff }}$ (incl.) selections, giving a total of 11 SRs. In order to estimate the backgrounds in a consistent and robust fashion, five control regions are defined for each of the SRs, giving 55 CRs in total. Each ensemble of one SR and five CRs constitutes a different "stream" of the analysis. The CR selections are optimized to maintain adequate statistical weight, while minimizing as far as possible the systematic uncertainties arising from extrapolation to the $\mathrm{SR}$, and any contamination from signal events. This is achieved by using kinematic selections that are as close as possible to the relevant SR, and making use of other event properties to create $\mathrm{CR}$ samples to measure the backgrounds.

The CRs are listed in Table II. CR1a and CR1b are used to estimate the contribution of $Z(\rightarrow \nu \bar{\nu})+$ jets background events to the SR by selecting samples of $\gamma+$ jets and $Z(\rightarrow \ell \ell)+$ jets events, respectively. The control region CR2 uses a reversed and tightened criterion on $\Delta \phi\left(\right.$ jet $_{i}$, $\left.\vec{P}_{\mathrm{T}}{ }^{\text {miss }}\right)_{\min }$ for up to three selected leading jets (depending on channel) to produce a data sample enriched with multijet background events. Otherwise it uses identical kinematic selections to the SRs. CR3 and CR4 use, respectively, a $b$-jet veto or $b$-jet requirement together with a lepton $+E_{\mathrm{T}}^{\text {miss }}$ transverse mass $\left(m_{\mathrm{T}}\right)$ requirement to select samples of $W(\rightarrow \ell \nu)+$ jets and semileptonic $t \bar{t}$ background events. Other selections are similar to those used to select the corresponding signal region, although in CR1b, CR3 and CR4 the requirements on $\Delta \phi\left(\right.$ jet $_{i}$, $\left.\vec{P}_{\mathrm{T}}{ }^{\text {miss }}\right)_{\min }$ and $E_{\mathrm{T}}^{\text {miss }} / m_{\text {eff }}$ are omitted to maximize the number of events without introducing extrapolations in energy or jet multiplicity.

The observed numbers of events in the CRs for each SR are used to generate internally consistent SM background estimates for the SR via a likelihood fit. This procedure enables CR correlations and contamination of the CRs by other SM processes and/or SUSY signal events to be taken into account. The same fit also allows the statistical significance of the observation in the SR with respect to the SM expectation to be determined. The estimated number of background events for a given process, $N(\mathrm{SR}$, scaled), is given by

$N(\mathrm{SR}$, scaled $)=N(\mathrm{CR}$, obs $) \times\left[\frac{N(\mathrm{SR}, \text { unscaled })}{N(\mathrm{CR}, \text { unscaled })}\right]$

where $N(\mathrm{CR}, \mathrm{obs})$ is the observed number of data events in the $\mathrm{CR}$ for the process, and $N(\mathrm{SR}$, unscaled) and $N(\mathrm{CR}$, unscaled) are estimates of the contributions from the process to the SR and CR, respectively, as described in Sec. IX. The ratio appearing in the square brackets in

TABLE II. Control regions used in the analysis: the main targeted background in the SR, the process used to model the background, and main CR selection(s) used to select this process are given.

\begin{tabular}{lccc}
\hline \hline CR & SR background & CR process & CR selection \\
\hline CR1a & $Z+$ jets & $\gamma+$ jets & Isolated photon \\
CR1b & $Z+$ jets & $Z(\rightarrow \ell \ell)+$ jets & $66 \mathrm{GeV}<m(\ell \ell)<116 \mathrm{GeV}$ \\
CR2 & Multijets & Multijets & $\Delta \phi\left(\text { jet }_{i}, \vec{P}_{\mathrm{T}}^{\text {miss }}\right)_{\min }<0.2 \mathrm{rad}$ \\
CR3 & $W(\rightarrow \ell \nu)+$ jets & $W(\rightarrow \ell \nu)+$ jets & $30 \mathrm{GeV}<m_{\mathrm{T}}\left(\ell, E_{\mathrm{T}}^{\text {miss }}\right)<100 \mathrm{GeV}, b$-veto \\
CR4 & $t \bar{t}$ and single top & $t \bar{t} \rightarrow b \bar{b} q q^{\prime} \ell \nu$ & $30 \mathrm{GeV}<m_{\mathrm{T}}\left(\ell, E_{\mathrm{T}}^{\text {miss }}\right)<100 \mathrm{GeV}, b$-tag \\
\hline \hline
\end{tabular}


Eq. (2) is defined to be the transfer factor (TF). Similar equations containing inter-CR TFs enable the background estimates to be normalized coherently across all the CRs. The likelihood fit adjusts the predicted background components in the CRs and SRs using the TFs and the unscaled $\mathrm{CR}$ event counts as constraints, taking into account their uncertainties. The scaled values are output from the fit.

The likelihood function for observing $n$ events in one of the channels (A-E, loose to tight) is the product of Poisson distributions, one for the signal region and one for each of the main control regions constraining the $Z+$ jets $(\mathrm{CR} 1 \mathrm{a} / \mathrm{b})$, multijets (CR2), $W+$ jets $(\mathrm{CR} 3)$ and $t \bar{t}$ (CR4) contributions, labeled $P_{\mathrm{SR}}, P_{\mathrm{ZRa}, \mathrm{b}}, P_{\mathrm{JR}}, P_{\mathrm{WR}}$ and $P_{\mathrm{TR}}$, respectively, and of the parton distribution functions (PDFs) constraining the systematic uncertainties $C_{\text {Syst }}$ :

$L(n \mid \mu, b, \theta)=P_{\mathrm{SR}} \cdot P_{\mathrm{ZRa}} \cdot P_{\mathrm{ZRb}} \cdot P_{\mathrm{JR}} \cdot P_{\mathrm{WR}} \cdot P_{\mathrm{TR}} \cdot C_{\mathrm{Syst}}(\theta)$.

The total expected background is $b$. The expected means for the Poisson distributions are computed from the observed numbers of events in the control regions, using the TFs. The signal strength $\mu$ parameterizes the expected signal, with $\mu=1$ giving the full signal expected in a given model. The nuisance parameters $(\theta)$ parameterize the systematic uncertainties, such as that on the integrated luminosity.

The expected number of events in the signal region is denoted by $\lambda_{S}$, while $\lambda_{i}$ denotes the expected number of events in control region $i$. These are expressed in terms of the fit parameters $\mu$ and $b$ and an extrapolation matrix $C$ (connecting background and signal regions) as follows:

$\lambda_{S}(\mu, b, \theta)=\mu \cdot C_{\mathrm{SR} \rightarrow \mathrm{SR}}(\theta) \cdot s+\sum_{j} C_{j \mathrm{R} \rightarrow \mathrm{SR}}(\theta) \cdot b_{j}$,

$\lambda_{i}(\mu, b, \theta)=\mu \cdot C_{\mathrm{SR} \rightarrow i \mathrm{R}}(\theta) \cdot s+\sum_{j} C_{j \mathrm{R} \rightarrow i \mathrm{R}}(\theta) \cdot b_{j}$,

where the index $j$ runs over the background control regions. The observed number of signal events in the $\mathrm{SR}\left(\mathrm{CR}_{j \mathrm{R}}\right)$ are $\mathrm{s}\left(b_{j}\right)$, respectively. The diagonal elements of the matrix are all unity by construction. The offdiagonal elements are the various TFs.

This background estimation procedure requires the determination of the central expected values of the TFs for each SM process, together with their associated correlated and uncorrelated uncertainties, as described in Sec. IX. The multijet TFs are estimated using a data-driven technique, which applies a resolution function to wellmeasured multijet events in order to estimate the effect of mismeasurement on $E_{\mathrm{T}}^{\text {miss }}$ and other variables. The other TF estimates use fully simulated MC samples validated with data (see Sec. IV B). Some systematic uncertainties, for instance those arising from the jet energy scale (JES), or theoretical uncertainties in MC simulation cross sections, largely cancel when calculating the event count ratios constituting the TFs.

The result of the likelihood fit for each stream includes a set of background estimates and uncertainties for the SR together with a $p$ value giving the probability for the hypothesis that the observed SR event count is compatible with background alone. Conservative assumptions are made about the migration of SUSY signal events between regions. When seeking an excess due to a signal in a particular SR, it is assumed that the signal contributes only to the SR; i.e. the SUSY TFs are all set to zero, giving no contribution from signal in the CRs. If no excess is observed, then limits are set within specific SUSY parameter spaces, taking into account theoretical and experimental uncertainties on the SUSY production cross section and kinematic distributions. Exclusion limits are obtained using a likelihood test. This compares the observed event rates in the signal regions with the fitted background expectation and expected signal contributions, for various signal hypotheses. Since the signal hypothesis for any specific model predicts the SUSY TFs, these exclusion limits do allow for signal contamination in the CRs.

\section{DATA AND SIMULATED SAMPLES}

\section{A. Proton-proton collision-data sample}

The data used in this analysis were taken in 2011 with the LHC operating at a center-of-mass energy of $7 \mathrm{TeV}$. Over this period the peak instantaneous luminosity increased from $1.3 \times 10^{30}$ to $3.7 \times 10^{33} \mathrm{~cm}^{-2} \mathrm{~s}^{-1}$ and the peak mean number of interactions per bunch crossing increased from 2 to 12. Application of beam, detector and data-quality requirements resulted in a total integrated luminosity of $4.7 \mathrm{fb}^{-1}[20,21]$. The precision of the luminosity measurement is $3.9 \%$. The trigger used is described in Sec. V.

\section{B. Monte Carlo samples}

MC samples are used to develop the analysis, optimize the selections, determine the transfer factors used to estimate the $W+$ jets, $Z+$ jets and top quark production backgrounds, and to assess sensitivity to specific SUSY signal models. Samples of simulated multijet events are generated with PYTHIA6 [22], using the MRST2007LO* modified leading-order PDFs [23], for use in the data-driven background estimates. Production of top quark pairs, including accompanying jets, is simulated with ALPGEN [24] and the CTEQ6L1 [25] PDF set, with a top quark mass of $172.5 \mathrm{GeV}$. Samples of $W$ and $Z / \gamma^{*}$ events with accompanying jets are also produced with ALPGEN. Diboson $\left(W W, W Z, Z Z, W \gamma^{*}\right)$ production is simulated with SHERPA [26]. Single top quark production is simulated with ACERMC [27]. Fragmentation and hadronization for the ALPGEN samples is performed with HERWIG [28,29], using JIMMY [30] for the underlying event. For the $\gamma+$ jet estimates of the $Z(\rightarrow \nu \bar{\nu})+$ jets backgrounds, photon and 
$Z$ events are also both produced using SHERPA for consistency checks of the ALPGEN results.

SUSY signal samples are generated with HERWIG++ [31] or MADGRAPH/PYTHIA6 [22,32,33]. Signal cross sections are calculated to next-to-leading order in the strong coupling constant, including the resummation of soft gluon emission at next-to-leading-logarithmic accuracy (NLO + NLL) [34-39]. The nominal cross section and the uncertainty are taken from an ensemble of cross section predictions using different PDF sets and factorization and renormalization scales, as described in Ref. [40].

The MC samples are generated using the same parameter set as Refs. [41-43] and passed through the ATLAS detector simulation [44] based on GEANT4 [45]. Differing pileup (multiple proton-proton interactions in a given event) conditions as a function of the LHC instantaneous luminosity are taken into account by overlaying simulated minimum-bias events onto the hard-scattering process and reweighting them according to the expected mean number of interactions per LHC bunch crossing.

\section{TRIGGER SELECTIONS}

The baseline triggers for the signal region event selection in the 2011 analysis use jets and $E_{\mathrm{T}}^{\text {miss }}[46,47]$. The jet and $E_{\mathrm{T}}^{\text {miss }}$ trigger required events to contain a leading jet with a transverse momentum $\left(p_{\mathrm{T}}\right)$, measured at the electromagnetic energy scale [48], above $75 \mathrm{GeV}$ and significant missing transverse momentum. The detailed trigger specification, including the value of the $E_{\mathrm{T}}^{\text {miss }}$ threshold, varied throughout the data-taking period, partly as a consequence of the rapidly increasing LHC luminosity. The trigger threshold on the missing transverse momentum increased from $45 \mathrm{GeV}$ at the start of the data-taking period to $55 \mathrm{GeV}$ at the end. The trigger reached its full efficiency of $>98 \%$ for events with a reconstructed jet with $p_{\mathrm{T}}$ exceeding $130 \mathrm{GeV}$ and more than $160 \mathrm{GeV}$ of missing transverse momentum. Trigger efficiencies are extracted using a sample selected by a looser trigger, taking into account correlations, i.e. correcting for the efficiency of the looser trigger. Prescaled single-jet triggers, which acquired fixed fractions of the data, are used for the trigger efficiency study.

A second study verifies that the efficiency of the baseline trigger becomes maximal at the values quoted above. The efficiencies are determined with an independent sample of events expected to possess $E_{\mathrm{T}}^{\text {miss }}$ generated by neutrinos. A sample triggered by electron candidates is used, where jets from electrons reconstructed with tight selection criteria are discarded. This trigger selected mostly $W \rightarrow e \nu$ events with jets and ran unprescaled, thus providing a large number of events.

\section{OBJECT RECONSTRUCTION}

The event reconstruction algorithms create the physics objects used in this analysis: electrons, muons, jets, photons and $b$ jets. Once these objects are defined, the overall missing transverse momentum can be calculated. A failure in the calorimeter electronics created a small dead region $(0<\eta<1.4,-0.8<\phi<-0.6)$ in the second and third layers of the electromagnetic calorimeter, which affected energy measurements in about $20 \%$ of the data sample. Any event with a jet that is inside the affected region and that is expected on the basis of shower shape to potentially contribute significantly to the $E_{\mathrm{T}}^{\text {miss }}$ is removed from the sample to avoid fake signals [49]. The energies of jets inside the affected region which are not expected to create $E_{\mathrm{T}}^{\text {miss }}$ are corrected using the functioning calorimeter layers.

Jet candidates are reconstructed using the anti- $k_{t}$ jet clustering algorithm [50,51] with a radius parameter of 0.4 . The inputs to this algorithm are clusters [52] of calorimeter cells seeded by those with energy significantly above the measured noise. Jet momenta are constructed by performing a four-vector sum over these cell clusters, measured at the electromagnetic scale, treating each as an $(E, \vec{p})$ four-vector with zero mass. The jet energies are corrected for the effects of calorimeter noncompensation and inhomogeneities by using $p_{\mathrm{T}^{-}}$and $\eta$-dependent calibration factors derived from MC simulation and validated with extensive test-beam and collision-data studies [53]. Only jet candidates with $p_{\mathrm{T}}>20 \mathrm{GeV}$ are subsequently retained.

Electron candidates are required to have $p_{\mathrm{T}}>20 \mathrm{GeV}$ and $|\eta|<2.47$ and to pass the "medium" electron shower shape and track selection criteria described in Ref. [54]. Muon candidates [55,56] are required to have matching tracks in the inner detector and muon spectrometer with $p_{\mathrm{T}}>10 \mathrm{GeV}$ and $|\eta|<2.4$.

Following the steps above, overlaps between candidate jets with $|\eta|<2.8$ and leptons are resolved as follows: first, any such jet candidate lying within a distance $\Delta R \equiv$ $\sqrt{(\Delta \eta)^{2}+(\Delta \phi)^{2}}=0.2$ ( $\phi$ measured in radians) of an electron is discarded; then any lepton candidate remaining within a distance $\Delta R=0.4$ of any surviving jet candidate is discarded. The first requirement prevents energy deposits from being interpreted as both jets and electrons. The second ensures that leptons produced within jets are not used to veto the event during the selection described in Sec. VIII.

The measurement of the missing transverse momentum two-vector $\vec{P}_{\mathrm{T}}$ miss is based on the transverse momenta of all remaining jet and lepton candidates and all calorimeter clusters not associated with such objects. Following this step, all jet candidates with $|\eta|>2.8$ are discarded, owing to their lower precision. Thereafter, the remaining lepton and jet candidates are considered "reconstructed," and the term "candidate" is dropped.

Photons are identified with the same selection criteria as used in the ATLAS prompt photon cross section analysis [57], where an isolated photon passing the tight photon identification criteria is required. Jets are classified as $b$ jets 
using a neural network algorithm, which takes as inputs the impact parameter measurements and the topological structure of $b$-quark decays, as described in Refs. [58,59].

\section{REMOVAL OF NONCOLLISION BACKGROUNDS}

Noncollision backgrounds are produced predominantly by noise sources in the calorimeters, cosmic ray events and beam collisions with residual gas in the beam pipe (beamgas events). The requirement of a vertex near the nominal interaction point with at least five associated tracks is effective at suppressing these backgrounds. Further criteria are applied which require that the fractional energy deposited in each calorimeter layer, and in any cells with known quality problems, is consistent with that expected from beam-beam events. In addition, the energy observed in charged particle tracks associated with the calorimeter cluster and the timing of the energy depositions in calorimeter cells with respect to the beam-crossing time are checked [53]. Following these selections, the remaining background is estimated by using the observed time distribution of the leading jets with respect to the bunch crossing, to create a background dominated control region. The noncollision background is found to be negligible in all of the SRs and CRs used.

\section{EVENT SELECTION}

Following the object reconstruction and event cleaning described above, a lepton veto is applied to reject $W(\rightarrow \ell \nu)+$ jets and leptonic $t \bar{t}$ events in which neutrinos generate the $E_{\mathrm{T}}^{\text {miss }}$ signature. The lepton $p_{\mathrm{T}}$ threshold used in the veto is set at 20 (10) $\mathrm{GeV}$ for electrons (muons) to ensure that selected events correspond to a phase-space region in which the veto efficiency is well understood.

TABLE III. Observed numbers of events in data and fitted background components in each SR. For the total background estimates, the quoted uncertainties give the statistical (MC simulation and CR combined) and systematic uncertainties, respectively. For the individual background components, the total uncertainties are given, while the values in parenthesis indicate the prefit predictions. The predictions for $W+$ jets, $Z+$ jets and $t \bar{t}$ plus single top quark are from ALPGEN and are normalized to luminosity. In the case of the multijet background, the prefit values are from the data-driven method, normalized at low $m_{\text {eff }}$. The diboson background is estimated with MC simulation normalized to luminosity. The $p$ values give the probability of the observation being consistent with the estimated background, and the "Gauss. $\sigma$ " values give the number of standard deviations in a Gaussian approximation, evaluated for a single observation at a time. The last two rows show the upper limits on the excess number of events, and the excess cross section, above that expected from the SM. The observed upper limit is followed in brackets by the expected limit, with the super- and subscripts showing the expectation from $\pm 1 \sigma$ changes in the background (denoted by $\uparrow$ and $\downarrow$, respectively).

\begin{tabular}{|c|c|c|c|c|c|c|}
\hline \multirow[b]{2}{*}{ Process } & \multicolumn{6}{|c|}{ Signal region } \\
\hline & SR-C loose & SR-E loose & SR-A medium & SR-A' medium & SR-C medium & SR-E medium \\
\hline$t \bar{t}+$ single top & $74 \pm 14(75)$ & $73 \pm 25(68)$ & $6.8 \pm 4.7(5.3)$ & $11 \pm 4(10)$ & $13 \pm 5(11)$ & $19 \pm 6(15)$ \\
\hline$Z+$ jets & $71 \pm 19(78)$ & $21 \pm 7(17)$ & $32 \pm 9(44)$ & $66 \pm 18(88)$ & $16 \pm 5(20)$ & $8.4 \pm 3.2(5.6)$ \\
\hline$W+$ jets & $61 \pm 11(61)$ & $23 \pm 13(23)$ & $19 \pm 5(21)$ & $25 \pm 5(30)$ & $7.7 \pm 3.0(11)$ & $6.2 \pm 2.6(4.7)$ \\
\hline Multijets & $0.9 \pm 1.2(0.8)$ & $8.4 \pm 7.3(25)$ & $0.1 \pm 0.3(0.2)$ & $0.0 \pm 0.1(0.5)$ & $0.03 \pm 0.05(0.03)$ & $1.4 \pm 1.2(2.7)$ \\
\hline Dibosons & $7.9 \pm 4.0(7.9)$ & $4.2 \pm 2.1(4.2)$ & $7.3 \pm 3.7(7.5)$ & $14 \pm 7(16)$ & $1.7 \pm 0.9(1.7)$ & $2.7 \pm 1.3(2.7)$ \\
\hline Total & $214 \pm 8 \pm 22$ & $129 \pm 8 \pm 30$ & $65 \pm 4 \pm 11$ & $116 \pm 5 \pm 19$ & $39 \pm 3 \pm 7$ & $38 \pm 4 \pm 5$ \\
\hline Data & 210 & 148 & 59 & 85 & 36 & 25 \\
\hline Local $p$ value (Gauss. $\sigma$ ) & $0.56(-0.15)$ & $0.21(0.81)$ & $0.66(-0.40)$ & $0.90(-1.3)$ & $0.61(-0.27)$ & $0.87(-1.1)$ \\
\hline Upper limit on $N_{\mathrm{BSM}}$ & $51\left(55_{\downarrow 76}^{\uparrow 42}\right)$ & $77\left(67_{\downarrow 91}^{\uparrow 49}\right)$ & $24\left(28_{\downarrow 39}^{\uparrow 20}\right)$ & $28\left(42_{\downarrow 58}^{\uparrow 31}\right)$ & $17\left(19_{\downarrow 26}^{\uparrow 14}\right)$ & $11\left(16_{\downarrow 23}^{\uparrow 12}\right)$ \\
\hline Upper limit on $\sigma(\mathrm{fb})$ & $11\left(12_{\downarrow 16}^{\uparrow 8.8}\right)$ & $16\left(14_{\downarrow 19}^{\uparrow 10}\right)$ & $5.1\left(5.9_{\downarrow 8.3}^{\uparrow 4.3}\right)$ & $6.0\left(8.9_{\downarrow 12}^{\dagger 6.6}\right)$ & $3.6\left(4_{\downarrow 5.6}^{\uparrow 2.9}\right)$ & $2.2\left(3.4_{\downarrow 4.8}^{\dagger 2.5}\right)$ \\
\hline
\end{tabular}

\begin{tabular}{lccccc}
\hline & & & Signal region & \\
Process & SR-A tight & SR-B tight & SR-C tight & SR-D tight & SR-E tight \\
\hline$t \bar{t}+$ single top & $0.2 \pm 0.2(0.1)$ & $0.3 \pm 0.3(0.2)$ & $2.0 \pm 1.5(1.2)$ & $2.4 \pm 1.7(1.4)$ & $4.2 \pm 4.7(3.0)$ \\
$Z+$ jets & $3.3 \pm 1.5(4.0)$ & $2.0 \pm 1.3(2.1)$ & $2.0 \pm 1.0(5.6)$ & $0.9 \pm 0.6(3.4)$ & $3.4 \pm 1.6(2.3)$ \\
$W+$ jets & $2.2 \pm 1.0(1.9)$ & $1.0 \pm 0.6(0.8)$ & $1.5 \pm 1.3(2.7)$ & $2.4 \pm 1.4(2.5)$ & $2.8 \pm 1.9(1.5)$ \\
Multijets & $0.00 \pm 0.02(0.01)$ & $0.00 \pm 0.07(0.02)$ & $0.00 \pm 0.03(0.01)$ & $0.0 \pm 0.3(0.1)$ & $0.5 \pm 0.4(0.9)$ \\
Dibosons & $1.8 \pm 0.9(2.0)$ & $1.8 \pm 0.9(1.9)$ & $0.5 \pm 0.3(0.5)$ & $2.2 \pm 1.1(2.2)$ & $2.5 \pm 1.3(2.5)$ \\
Total & $7.4 \pm 1.3 \pm 1.9$ & $5.0 \pm 0.9 \pm 1.7$ & $6.0 \pm 1.0 \pm 2.0$ & $7.8 \pm 1.0 \pm 2.4$ & $13 \pm 2 \pm 6$ \\
Data & 1 & 1 & 14 & 9 & 13 \\
Local $p$ value $(\mathrm{Gauss} . \sigma)$ & $0.98(-2.1)$ & $0.96(-1.7)$ & $0.016(2.1)$ & $0.29(0.55)$ & $0.45(0.14)$ \\
Upper limit on $N_{\mathrm{BSM}}$ & $3.1\left(6.4_{\downarrow 9.4}^{\dagger 4.5}\right)$ & $3.0\left(5.6_{\downarrow 8.3}^{\dagger 3.9}\right)$ & $16\left(9.5_{\downarrow 14}^{\dagger 6.9}\right)$ & $9.6\left(8.5_{\downarrow 12}^{\dagger 6.1}\right)$ & $12\left(12_{\downarrow 17}^{\dagger 8.4}\right)$ \\
Upper limit on $\sigma(\mathrm{fb})$ & $0.66\left(1.4_{\downarrow 2.0}^{\dagger 0.96}\right)$ & $0.64\left(1.2_{\downarrow 1.8}^{\dagger 0.83}\right)$ & $3.4\left(2.0_{\downarrow 2.9}^{\dagger 1.5}\right)$ & $2.0\left(1.8_{\downarrow 2.6}^{\dagger 1.3}\right)$ \\
\hline \hline
\end{tabular}


The signal regions are then defined by the kinematic selections given in Table I. Requirements on the transverse momenta of additional jets select inclusive 2-, 3-, 4-, 5- and 6-jet events in channels A/ $\mathrm{A}^{\prime}, \mathrm{B}, \mathrm{C}, \mathrm{D}$ and $\mathrm{E}$, respectively. The jet $p_{\mathrm{T}}$ thresholds for the leading up to four jets are set at $60 \mathrm{GeV}$ in order to minimize the impact of pileup on selection efficiency and improve background rejection.

Removing events with a small angle in the transverse plane $(\Delta \phi)$ between jets and $E_{\mathrm{T}}^{\text {miss }}$ suppresses multijet

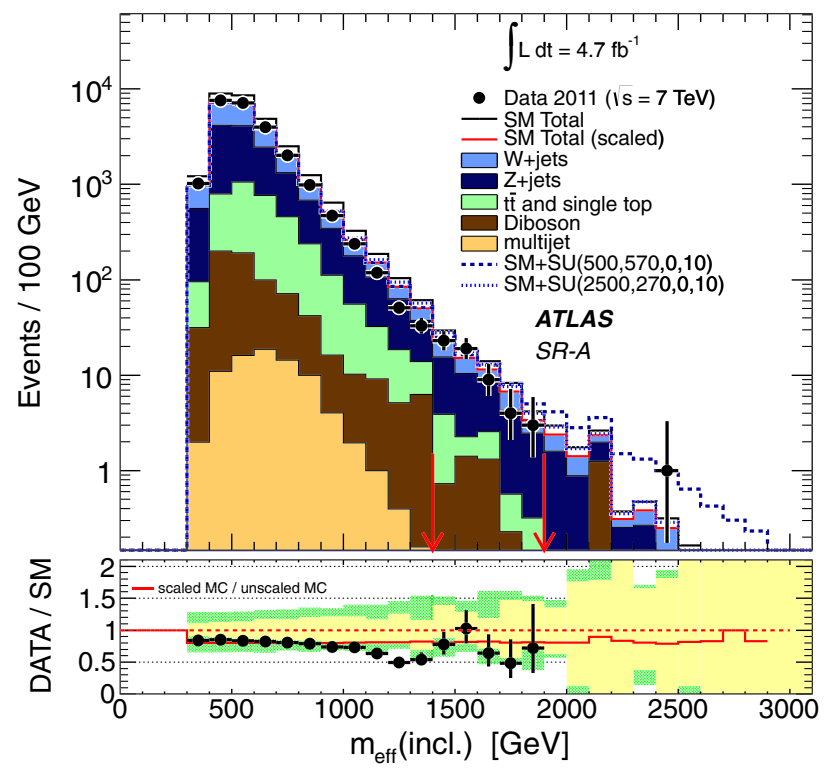

FIG. 1 (color online). Observed $m_{\text {eff }}$ (incl.) distribution for channel A. In the top panel, the histograms show the SM background expectations, both before (black open histogram) and after [medium (red) open histogram] use of a fit to scale the expectations to CR observations. This fit is applied to illustrate the SR + CR fitting technique used in the main analysis. Before scaling, the MC simulation expectations are normalized to luminosity. The multijet background is estimated using the jet smearing method described in the text. After scaling, the $W+$ jets, $Z+$ jets and $t \bar{t}$ and single top quark and multijet distributions (denoted by full histograms) are normalized to data in corresponding control regions over the full $m_{\text {eff }}$ range. Two MSUGRA/CMSSM benchmark model points with $m_{0}=$ $500 \mathrm{GeV}, m_{1 / 2}=570 \mathrm{GeV}, A_{0}=0, \tan \beta=10$ and $\mu>$ and with $m_{0}=2500 \mathrm{GeV}, m_{1 / 2}=270 \mathrm{GeV}, A_{0}=0, \tan \beta=10$ and $\mu>$, illustrating different topologies, are also shown. These points lie just beyond the reach of the previous analysis [17]. The arrows indicate the locations of the lower edges of the two signal regions. The bottom panel shows the fractional deviation of the data from the total unscaled background estimate (black points), together with the fractional deviation of the total scaled background estimate from the total unscaled background estimate [medium (red) line]. The light (yellow) band shows the combined experimental uncertainties on the unscaled background estimates from jet energy scale, jet energy resolution, the effect of pileup, the treatment of energy outside of reconstructed jets and MC simulation sample size. The medium (green) band includes also the total theoretical uncertainties.

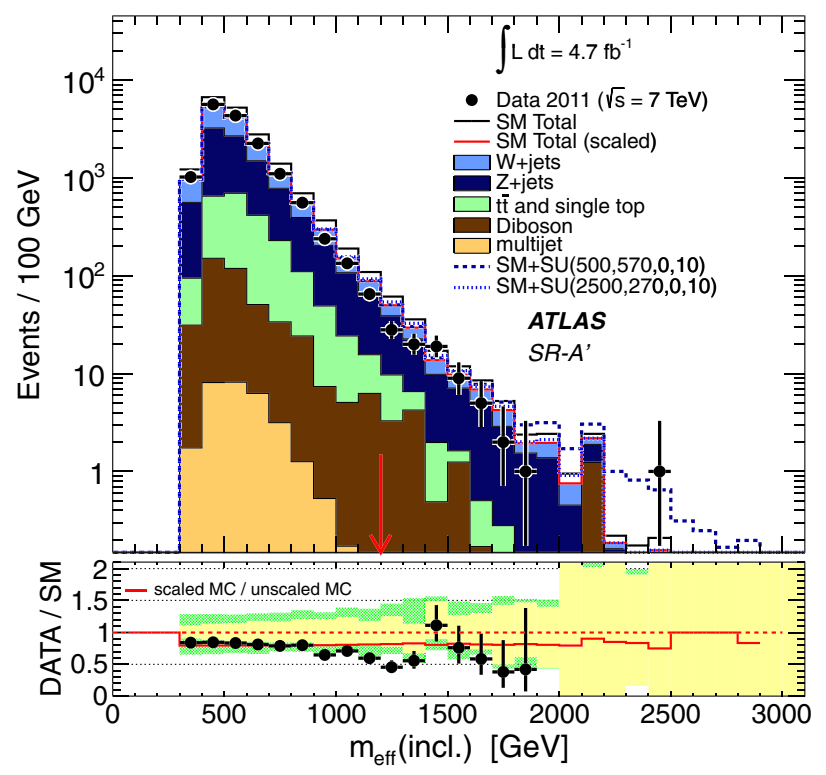

FIG. 2 (color online). Observed $m_{\text {eff }}$ (incl.) distribution for channel $\mathrm{A}^{\prime}$, as for Fig. 1.

background in which mismeasurement of jet energy generates fake missing transverse momentum along the jet direction. For channels $\mathrm{A}, \mathrm{A}^{\prime}$ and $\mathrm{B}$ a requirement $\Delta \phi>$ 0.4 radians is applied to the leading (up to) three selected jets with $p_{\mathrm{T}}>40 \mathrm{GeV}$, before the final SR selection, to minimize loss of signal efficiency. For the other channels this requirement is augmented by a looser requirement that $\Delta \phi>0.2 \mathrm{rad}$ for all remaining selected jets with $p_{\mathrm{T}}>40 \mathrm{GeV}$.

Multijet background is further suppressed by requiring that the $E_{\mathrm{T}}^{\text {miss }}$ exceeds a specific fraction of the effective

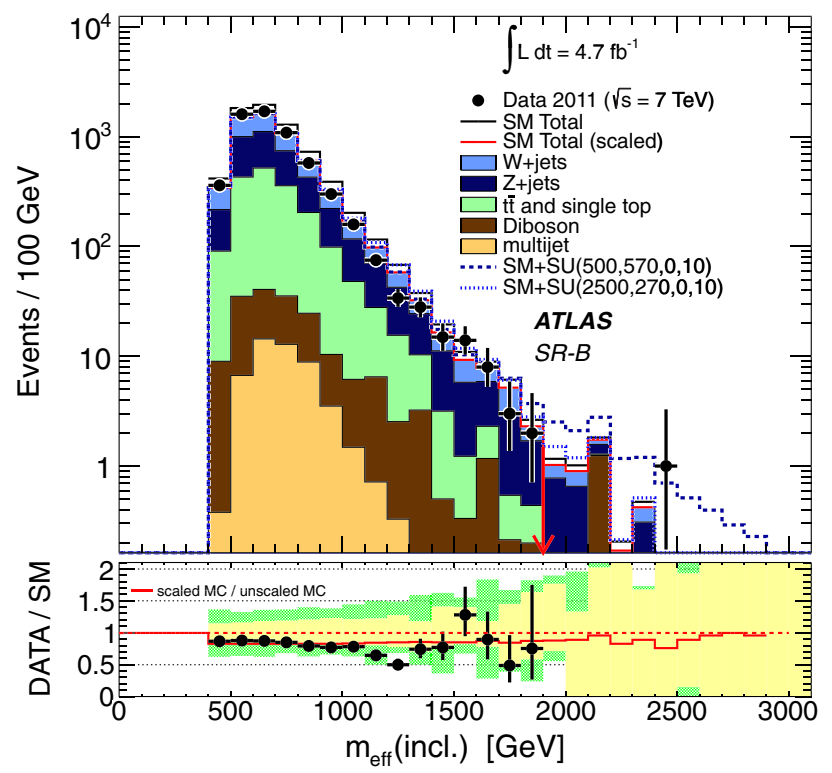

FIG. 3 (color online). Observed $m_{\text {eff }}$ (incl.) distribution for channel B, as for Fig. 1. 


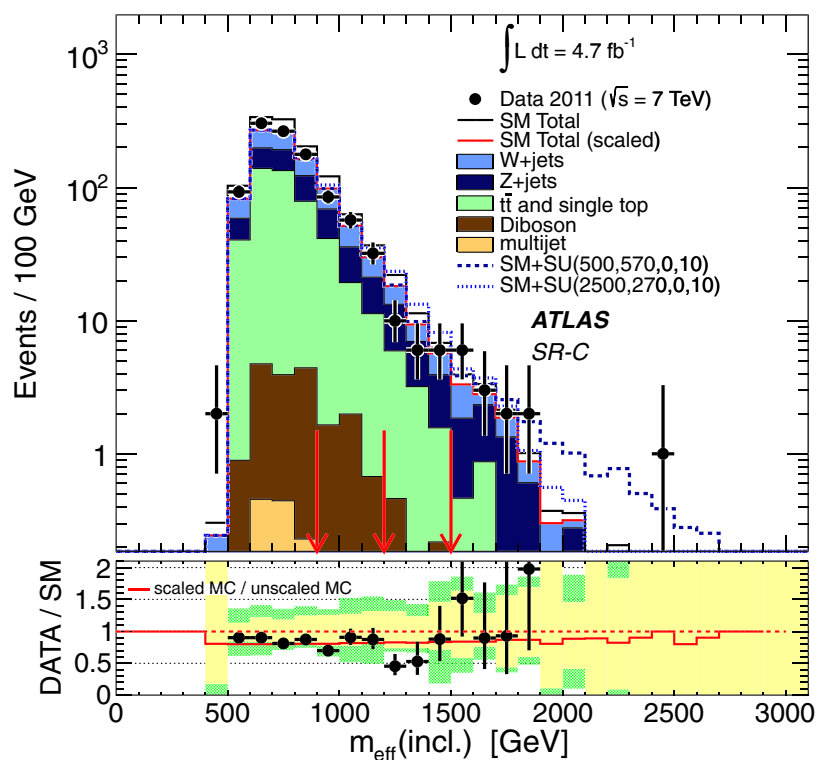

FIG. 4 (color online). Observed $m_{\text {eff }}$ (incl.) distribution for channel $\mathrm{C}$, as for Fig. 1.

mass of the event, $m_{\text {eff }}$. Coupled with the explicit requirement on $m_{\text {eff }}$ (incl.) discussed below this equates to a hard selection on $E_{\mathrm{T}}^{\text {miss }}$. The $E_{\mathrm{T}}^{\text {miss }} / m_{\text {eff }}$ value used decreases with increasing jet multiplicity because the typical $E_{\mathrm{T}}^{\text {miss }}$ of SUSY signal events is inversely correlated with jet multiplicity due to phase-space limitations. This is because additional jets in a SUSY decay chain increase the probability that the lightest SUSY particle (LSP) will be produced with low momentum through effective multibody decays. Small mass splittings can also lead to low $E_{\mathrm{T}}^{\text {miss }}$. The multijet cross section is also suppressed at higher jet

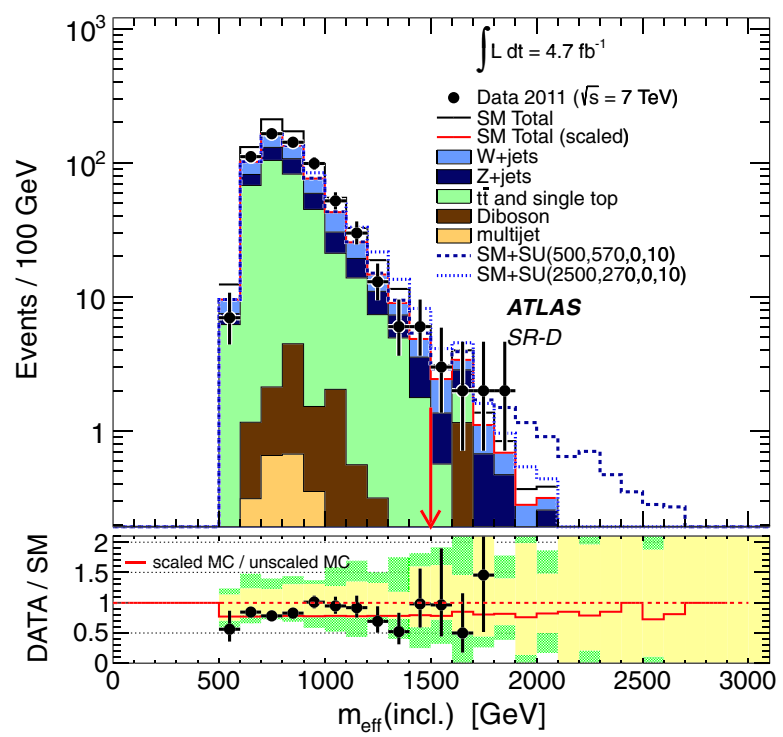

FIG. 5 (color online). Observed $m_{\text {eff }}$ (incl.) distribution for channel D, as for Fig. 1.

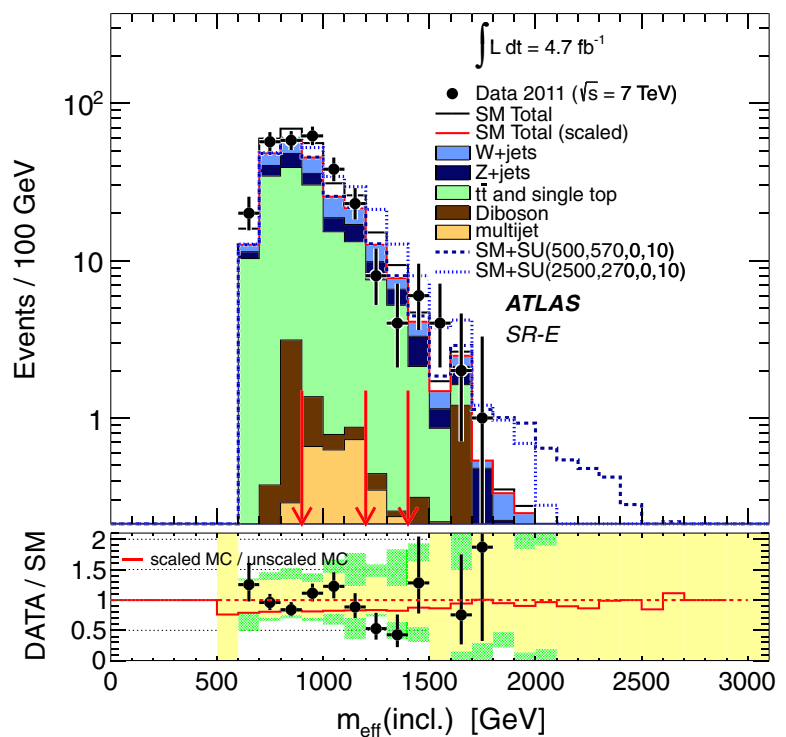

FIG. 6 (color online). Observed $m_{\mathrm{eff}}$ (incl.) distribution for channel E, as for Fig. 1.

multiplicities, allowing the $E_{\mathrm{T}}^{\text {miss }}$ requirement to be loosened.

Finally, the signal regions are defined by criteria on $m_{\text {eff }}$ (incl.) which select events with hard kinematics in order to provide strong suppression of all SM background processes. Up to three $m_{\text {eff }}$ (incl.) values are specified per channel, corresponding to distinct signal regions tight, medium and loose, in which the final event samples are counted.

Table III lists the number of data events passing each of the SR selections. The distributions of $m_{\text {eff }}$ (incl.) [prior to the final $m_{\text {eff }}$ (incl.) selections] for each channel for data and SM backgrounds are shown in Figs. 1-6. Details of the CR selections, and the methods used to obtain the background estimates follow in Sec. IX. The information is used in Sec. XI to produce the final results.

\section{BACKGROUND ESTIMATION}

\section{A. Introduction}

The $Z(\rightarrow \nu \bar{\nu})+$ jets process constitutes the dominant irreducible background in this analysis. It is estimated using control regions enriched in related processes with similar kinematics: events with isolated photons and jets [60] (CR1a, Sec. IX B) and $Z(\rightarrow e e / \mu \mu)+$ jets events (CR1b, Sec. IX C). The reconstructed momentum of the photon or the lepton-pair system is added to $\vec{P}_{\mathrm{T}}$ miss to obtain an estimate of the $E_{\mathrm{T}}^{\text {miss }}$ observed in $Z(\rightarrow \nu \bar{\nu})+$ jets events. The predictions from both control regions are found to be in good agreement, and both are used in the final fit. The small additional background contributions from $Z(\rightarrow e e / \mu \mu / \tau \tau)$ decays in which the leptons are misidentified or unreconstructed, and from misidentified photon events, are estimated using the same control regions 
with appropriate transfer factors. The TF for CR1a estimates $Z(\rightarrow \nu \bar{\nu})+$ jets in the SR and is corrected to give an estimate of $Z+$ jets in the SR by multiplying by the ratio of $Z+$ jets events to $Z(\rightarrow \nu \bar{\nu})+$ jets events derived from MC simulation. In the case of $\mathrm{CR} 1 \mathrm{~b}$ the $\mathrm{TF}$ is calculated between $Z(\rightarrow e e / \mu \mu / \tau \tau)+$ jets in the $\mathrm{CR}$ and $Z(\rightarrow$ $\nu \bar{\nu} / e e / \mu \mu / \tau \tau)+$ jets in the SR. Thus both methods ultimately provide an estimate of the total $Z+$ jets background in the SR.

The backgrounds from multijet processes are estimated using a data-driven technique based upon the convolution of jets in a low $E_{\mathrm{T}}^{\text {miss }}$ data sample with jet response functions derived from multijet dominated data control regions (Sec. IX D). Those from $W+$ jets and top quark processes are derived from MC simulation (Sec. IXE).

For each stream a likelihood fit is performed to the observed event counts in the five CRs, taking into account correlations in the systematic uncertainties in the transfer factors.

\section{B. $Z+$ jets estimate using a $\gamma+$ jets control region}

The magnitude of the irreducible background from $Z(\rightarrow \nu \bar{\nu})+$ jets events in the SRs can be estimated using $\gamma+$ jets data. When the vector boson $p_{\mathrm{T}}$ is large, as required by the SR selections, the $Z$ and $\gamma$ cross sections differ mainly by their coupling constants with respect to quarks. For this reason the cross section ratio

$$
R_{Z / \gamma}=\frac{d \sigma(Z+\text { jets }) / d p_{\mathrm{T}}}{d \sigma(\gamma+\text { jets }) / d p_{\mathrm{T}}}
$$

can be used to translate the observed number of photon events in the CR into an estimate of the number of $Z$ events in the SR, taking into account the leptonic branching ratios of the $Z$ and other effects. The ratio is expected to be robust with respect to both theoretical uncertainties and experimental effects, related to, for example, jet reconstruction, which would be similar for both processes and therefore cancel in the ratio.

The method uses photon events which are selected in two steps. The first aims to select a photon event sample where the efficiency and the background contamination are well known. The SR selections are then applied to these photon events, having added the photon $p_{\mathrm{T}}$ to the $E_{\mathrm{T}}^{\text {miss }}$ of the event to reproduce the $E_{\mathrm{T}}^{\text {miss }}$ observed in $Z(\rightarrow \nu \bar{\nu})$ background events. The SR selections consist primarily of requirements on the jets and $E_{\mathrm{T}}^{\text {miss }}$ in the event, which directly or indirectly, due to the $p_{\mathrm{T}}$ recoil, impose kinematic constraints on the vector boson, i.e. the $Z$ or photon.

Photon events are selected by requiring at least one isolated photon passing the photon identification criteria discussed above. The photon trigger has an efficiency close to $100 \%$ for selected events with a photon $p_{\mathrm{T}} \geq 85 \mathrm{GeV}$. The photons are required to lie within the fiducial region $|\eta|<1.37$ and $1.52 \leq|\eta|<2.37$. After this first photon event selection a total of $2.8 \mathrm{M}$ photon candidates are obtained from the complete data set, with an estimated purity $>95 \%$. Figure 7 (a) shows the leading photon $p_{\mathrm{T}}$ distribution for events passing the first photon selection.

In the second selection step, the SR selection criteria from Table I are applied to the photon sample. In order to prevent the reconstructed photon in the event from also being reconstructed as a jet, jets within $\Delta R=0.2$ of the photon are removed. The photon $p_{\mathrm{T}}$ is added to the $E_{\mathrm{T}}^{\text {miss }}$ vectorial sum when applying the SR selections, using the appropriate calibration for the electromagnetic character of the photon shower.

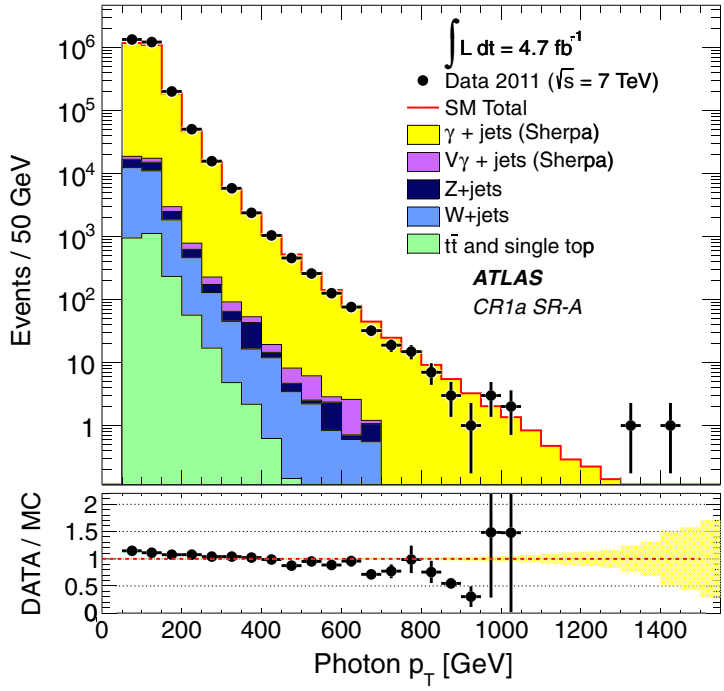

(a)

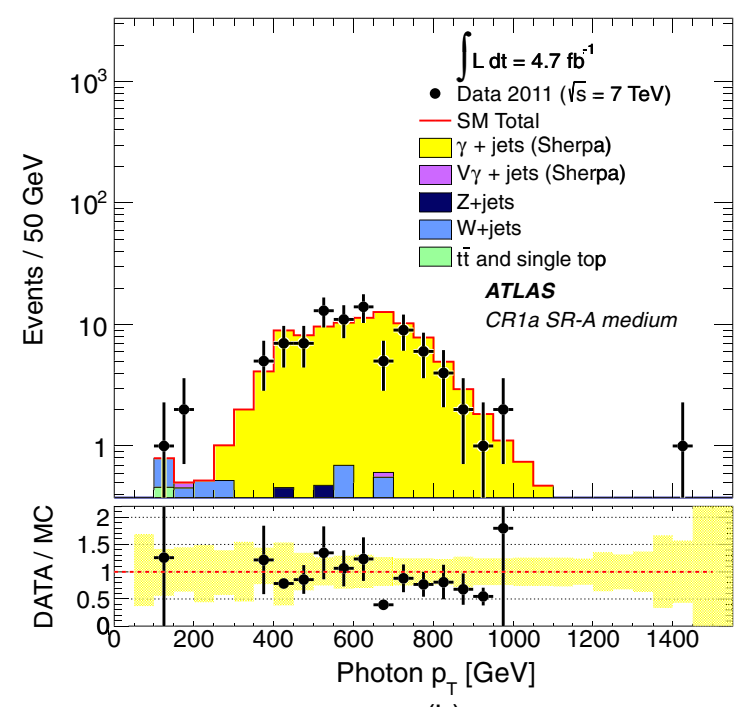

(b)

FIG. 7 (color online). Leading photon $p_{\mathrm{T}}$ distribution from data and MC simulation (a) directly after the photon selection and (b) in CR1a for SR-A medium that requires $m_{\text {eff }}>1400 \mathrm{GeV}$. The bottom panel shows the ratio of data to MC expectation, with the light (yellow) band indicating the uncertainty. 
TABLE IV. Numbers of photon events observed in the data and expected from the SHERPA and ALPGEN MC simulations in CR1a for each SR, as well as the resulting estimated numbers of $Z(\rightarrow \nu \bar{\nu})$ events in the SRs, with statistical and systematic uncertainties.

\begin{tabular}{|c|c|c|c|c|}
\hline SR & $\begin{array}{l}\text { Minimum } \\
m_{\mathrm{eff}} \mathrm{GeV}\end{array}$ & $\begin{array}{c}\gamma \mathrm{CR} 1 \mathrm{a} \\
\text { data }\end{array}$ & $\begin{array}{c}\gamma \mathrm{CR} 1 \mathrm{a} \mathrm{MC} \\
\text { SHERPA/ALPGEN }\end{array}$ & $\begin{array}{c}\text { Est. } Z_{\nu \nu} \\
\operatorname{SR}(\gamma) \text { data }\end{array}$ \\
\hline \multirow[t]{2}{*}{ A } & 1400 & 90 & $96 / 93.4$ & $32.0 \pm 3.4 \pm 5.6$ \\
\hline & 1900 & 9 & $9.42 / 9.33$ & $3.2 \pm 1.1 \pm 0.6$ \\
\hline $\mathrm{A}^{\prime}$ & 1200 & 170 & $176 / 180$ & $62 \pm 5 \pm 11$ \\
\hline B & 1900 & 5 & $6.21 / 6.31$ & $1.9 \pm 0.8 \pm 0.4$ \\
\hline \multirow[t]{3}{*}{$\mathrm{C}$} & 900 & 223 & $219 / 197$ & $64 \pm 4 \pm 11$ \\
\hline & 1200 & 48 & $55.8 / 44.5$ & $15 \pm 2 \pm 3$ \\
\hline & 1500 & 6 & $14.4 / 11.1$ & $1.9 \pm 0.8 \pm 0.4$ \\
\hline $\mathrm{D}$ & 1500 & 3 & $10.9 / 6.98$ & $0.86 \pm 0.50 \pm 0.24$ \\
\hline \multirow[t]{3}{*}{$\mathrm{E}$} & 900 & 77 & $71.5 / 47.4$ & $20 \pm 2 \pm 5$ \\
\hline & 1200 & 26 & $15.3 / 13.9$ & $7.7 \pm 1.5 \pm 1.9$ \\
\hline & 1400 & 11 & $8.71 / 6.11$ & $3.4 \pm 1.0 \pm 1.0$ \\
\hline
\end{tabular}

The numbers of photon candidates which are selected by the CR1a criteria for channels A-E are presented in Table IV together with the numbers expected from MC simulation. Figure 7(b) shows the leading photon $p_{\mathrm{T}}$ distribution for events in CR1a for SR-A medium, that requires $m_{\text {eff }}>1400 \mathrm{GeV}$. Good agreement is seen between the data and the MC simulation.

These numbers of photons are corrected for experimental effects as described in Ref. [57] before being used to estimate the TFs. The following effects are considered. The combined identification and reconstruction efficiency is estimated to be $86 \%$, with an uncertainty of less than $1 \%$. The identification inefficiency is dominated by the tight photon identification requirements and decreases with increasing photon $p_{\mathrm{T}}$. A further uncertainty of $5 \%$ is included to account for differences in efficiency of the photon isolation criteria in different event samples. Backgrounds from multijet processes and $W+$ jets events where an electron from the $W$ decay is misidentified as a photon are each estimated to be $\sim 1 \%$ for $p_{\mathrm{T}}^{\gamma}>200 \mathrm{GeV}$. Therefore the background is neglected, but an uncertainty of $5 \%$ is assigned.

The number of photon events selected by the CR1a criteria is used to estimate the expected number of $Z(\rightarrow \nu \bar{\nu})$ events in the corresponding SR using

$$
\begin{aligned}
N^{Z(\rightarrow \bar{\nu})}\left(p_{\mathrm{T}}\right)= & N^{\gamma}\left(p_{\mathrm{T}}\right) \cdot\left[\frac{\left(1-f_{\mathrm{bkg}}\right)}{\varepsilon^{\gamma}\left(p_{\mathrm{T}}\right) \cdot A^{\gamma}\left(p_{\mathrm{T}}\right)}\right. \\
& \left.\cdot R_{Z / \gamma}\left(p_{\mathrm{T}}\right) \cdot \operatorname{Br}(Z \rightarrow \nu \bar{\nu})\right] .
\end{aligned}
$$

Here $N^{\gamma}\left(p_{\mathrm{T}}\right)$ represents the number of photon candidate events passing the CR1a selections, binned in $p_{\mathrm{T}}$ as in Fig. 7(b), $f_{\text {bkg }}$ the fraction of fake photons in the control region, $\varepsilon^{\gamma}\left(p_{\mathrm{T}}\right)$ the efficiency for selecting the photons and $A^{\gamma}\left(p_{\mathrm{T}}\right)$ the photon acceptance. The cross section ratio $R_{Z / \gamma}\left(p_{\mathrm{T}}\right)$ is determined from MC simulation. The uncertainties related to the cross section ratio have been studied using the two MC programs PYTHIA8 [61] and GAMBOS (an adaptation of the VECBOS program [60,62]) and many of the theoretical uncertainties, such as the choice of scales and parton distribution functions, are found to cancel in the ratio, to a large extent [60]. It has, however, been shown that the ratio retains slight sensitivity to the jet selection and that multiparton matrix elements must be used to describe correctly all the relevant amplitudes. The final uncertainties on $R_{Z / \gamma}\left(p_{\mathrm{T}}\right)$ should therefore be small, but a

TABLE V. Summary of transfer factors from the main control regions of each background component in every stream. In CR4 for signal regions A tight and B tight the $m_{\text {eff }}$ requirements were relaxed to $1500 \mathrm{GeV}$ to increase the numbers of events in the CRs for the

\begin{tabular}{|c|c|c|c|c|c|}
\hline Signal region & $\mathrm{CR} 1 \mathrm{a} / Z+$ jets & $\mathrm{CR} 1 \mathrm{~b} / Z+$ jets & $\begin{array}{r}\text { Control region/p } \\
\text { CR2/Multijets }\end{array}$ & $\mathrm{CR} 3 / W+$ jets & $\mathrm{CR} 4 / t \bar{t}+$ single top \\
\hline SR-C loose & $0.32_{-0.09}^{+0.08}$ & $2.9_{-0.4}^{+0.7}$ & $0.016_{-0.012}^{+0.012}$ & $0.36_{-0.05}^{+0.04}$ & $0.52_{-0.08}^{+0.08}$ \\
\hline SR-E loose & $0.27_{-0.08}^{+0.08}$ & $6.5_{-3.0}^{+5.0}$ & $0.05_{-0.04}^{+0.04}$ & $0.74_{-0.13}^{+0.12}$ & $0.92_{-0.19}^{+0.18}$ \\
\hline SR-A medium & $0.36_{-0.10}^{+0.10}$ & $2.5_{-1.0}^{+0.7}$ & $0.032_{-0.019}^{+0.019}$ & $0.31_{-0.05}^{+0.05}$ & $0.34_{-0.22}^{+0.22}$ \\
\hline SR-A' medium & $0.39_{-0.10}^{+0.10}$ & $2.2_{-0.6}^{+0.5}$ & $0.10_{-0.06}^{+0.06}$ & $0.19_{-0.02}^{+0.03}$ & $0.23_{-0.07}^{+0.06}$ \\
\hline SR-C medium & $0.34_{-0.10}^{+0.09}$ & $2.9_{-1.0}^{+1.8}$ & $0.003_{-0.001}^{+0.005}$ & $0.20_{-0.05}^{+0.06}$ & $0.30_{-0.10}^{+0.10}$ \\
\hline SR-E medium & $0.32_{-0.10}^{+0.10}$ & $5.0_{-3.0}^{+9.0}$ & $0.038_{-0.031}^{+0.031}$ & $0.39_{-0.10}^{+0.10}$ & $0.62_{-0.19}^{+0.17}$ \\
\hline SR-A tight & $0.30_{-0.08}^{+0.08}$ & $5.3_{-3.7}^{+4.1}$ & $0.009_{-0.009}^{+0.009}$ & $0.25_{-0.10}^{+0.09}$ & $0.01_{-0.02}^{+0.02}$ \\
\hline SR-B tight & $0.38_{-0.10}^{+0.10}$ & $4.2_{-5.3}^{+3.7}$ & $0.011_{-0.008}^{+0.008}$ & $0.14_{-0.08}^{+0.07}$ & $0.022_{-0.024}^{+0.023}$ \\
\hline SR-C tight & $0.32_{-0.09}^{+0.09}$ & $1.8_{-0.9}^{+1.4}$ & $0.0034_{-0.0025}^{+0.0044}$ & $0.16_{-0.11}^{+0.11}$ & $0.15_{-0.13}^{+0.12}$ \\
\hline SR-D tight & $0.29_{-0.08}^{+0.10}$ & $2.1_{-2.8}^{+4.8}$ & $0.02_{-0.01}^{+0.01}$ & $0.26_{-0.10}^{+0.10}$ & $0.20_{-0.16}^{+0.15}$ \\
\hline SR-E tight & $0.31_{-0.10}^{+0.11}$ & $2.7_{-4.5}^{+2.8}$ & $0.04_{-0.02}^{+0.02}$ & $0.26_{-0.08}^{+0.10}$ & $0.32_{-0.25}^{+0.27}$ \\
\hline
\end{tabular}
minor $t \bar{t}$ background. 
conservative uncertainty of $25 \%$ is assigned. Additional systematic uncertainties, common to several parts of the analysis, are discussed in Sec. X.

The transfer factors between the CR1a regions and their associated signal regions are obtained by averaging the correction term in the square brackets of Eq. (7) over the measured $p_{\mathrm{T}}$ distribution of selected photon candidates and are given in Table $\mathrm{V}$.

\section{C. $Z+$ jets estimate using a $Z(\rightarrow \ell \ell)+$ jets control region}

The irreducible background from $Z(\rightarrow \nu \bar{\nu})+$ jets can also be estimated independently using the observed leptonic $Z$ decays. The CR1b control regions are defined by requiring two opposite-sign electrons or muons with $p_{\mathrm{T}}>$ $20 \mathrm{GeV}$. In addition, the $p_{\mathrm{T}}$ of the leading electron is required to be above $25 \mathrm{GeV}$ to protect against trigger turn-on effects. The dilepton invariant mass must lie in the range $66 \mathrm{GeV}<m(\ell \ell)<116 \mathrm{GeV}$. The $E_{\mathrm{T}}^{\text {miss }}$ variable in the SR selection is emulated with the vectorial sum of the reconstructed $Z$ boson momentum vector and the measured $\vec{P}_{\mathrm{T}}{ }^{\text {miss }}$. The SR jet and $E_{\mathrm{T}}^{\text {miss }}$ requirements are applied, without selections on $\Delta \phi\left(\text { jet }_{i}, \vec{P}_{\mathrm{T}}{ }^{\text {miss }}\right)_{\min }$ or $E_{\mathrm{T}}^{\text {miss }} / m_{\text {eff }}$. These changes are made to increase the acceptance, since the precision of the method is limited by the rate of dilepton events.

In order to calculate the transfer factors, ALPGEN is used to estimate the number of $Z+$ jets events in each SR and the number of $Z(\rightarrow \ell \ell)+$ jets events in each corresponding CR1b control region. The uncertainties arise from the number of MC simulation events, the jet energy scale and resolution, the electron and muon energy resolutions, the electron and muon selection efficiencies, the electron trigger efficiency, the electron energy scale, energy included in calorimeter clusters that is not associated with physics objects, the input PDFs, the modeling of pileup in the simulation, and the luminosity.

The transfer factors themselves are listed in Table V and take into account the contribution from leptonic $Z(\rightarrow \tau \tau)+$ jets events in CR1b. The estimated numbers of $Z+$ jets events obtained using this technique are consistent with those estimated using $\gamma+$ jets events observed in CR1a.

\section{Multijet background estimation}

The probability for multijet events to pass any of the SR selection cuts used in this analysis is, by design, very small. However, the large cross section for this process could potentially compensate for the low acceptance and hence lead to significant SR contamination. These two effects also limit the applicability of conventional MC simulation techniques; firstly because very large MC data samples are required and secondly because accurate modeling of the acceptance requires exceptionally detailed understanding of the performance of every component of the calorimeters. For this reason a data-driven method is used to estimate the multijet background in the SRs. The method makes use of high-statistics samples of well-measured data multijet events to minimize statistical uncertainties. In order to determine the acceptance of the SRs for poorly measured multijet events, the jets in these events are convoluted with a function modeling the response of the calorimeters. This response function is based upon the results of MC simulations but is modified in such a way as to give good agreement between multijet estimates and data in two additional dedicated analyses. This procedure minimizes the susceptibility of the multijet background estimates in the main analysis to systematic uncertainties arising from the Monte Carlo modeling of the initial response function.

The jet response function quantifies the probability of fluctuation of the measured $p_{\mathrm{T}}$ of jets and takes into account both the effects of jet mismeasurement and contributions from neutrinos and muons in jets from heavy flavor decays. This function is convoluted with the fourvectors of jets in low- $E_{\mathrm{T}}^{\text {miss }}$ multijet data events, generating higher $E_{\mathrm{T}}^{\mathrm{miss}}$ events. These are referred to as "pseudodata" and are used to provide a minimally MC simulation dependent estimate of multijet distributions, including the distribution of $\Delta \phi\left(\text { jet }_{i}, \vec{P}_{\mathrm{T}}{ }^{\text {miss }}\right)_{\min }$ for high $m_{\mathrm{eff}}$ events. These distributions can be used to determine the transfer factors from the low $\Delta \phi\left(\text { jet }_{i}, \vec{P}_{\mathrm{T}}{ }^{\text {miss }}\right)_{\text {min }}$ multijet control regions CR2 to the higher $\Delta \phi\left(\text { jet }_{i}, \vec{P}_{\mathrm{T}}{ }^{\text {miss }}\right)_{\text {min }}$ signal regions.

The method, referred to as the "jet smearing method" below, proceeds in four steps:

(1) Selection of low- $E_{\mathrm{T}}^{\text {miss }}$ seed events in the data. The jets in these events are well measured. These events are used in steps (3) and (4).

(2) As a starting point the response function is determined in MC simulated data by comparing generator-level jet energy to reconstructed detector-level jet energy.

(3) Jets in the seed events are convoluted with the response function to generate pseudodata events. The consistency between pseudodata and experimental data in two analyses (see below) is then determined. The response function is modified and the convolution repeated until good agreement is obtained.

(4) Jets in the seed events are convoluted with the resulting data-constrained response function to obtain a final sample of pseudodata events. This sample is used to estimate the distributions of variables defining the control and signal regions used in the main analysis.

Seed events are triggered using single-jet triggers and offline thresholds of 50,100,130,165, 200, 260 and $335 \mathrm{GeV}$ are then applied. To ensure that the events contain only well-measured jets, the $E_{\mathrm{T}}^{\text {miss }}$ significance (defined as $E_{\mathrm{T}}^{\text {miss }} / \sqrt{E_{\mathrm{T}}^{\text {sum }}}$, where $E_{\mathrm{T}}^{\text {sum }}$ is the scalar sum of the transverse 
energy measured in the calorimeters) is required to be $<0.6 \mathrm{GeV}^{1 / 2}$.

The response function is initially estimated from MC simulation by matching "truth" jets reconstructed from generator-level particles to detector-level jets with $\Delta R<$ 0.1 in multijet samples. The four-momenta of any generator-level neutrinos in the truth jet cone are added to the four-momentum of the truth jet. Truth jets are

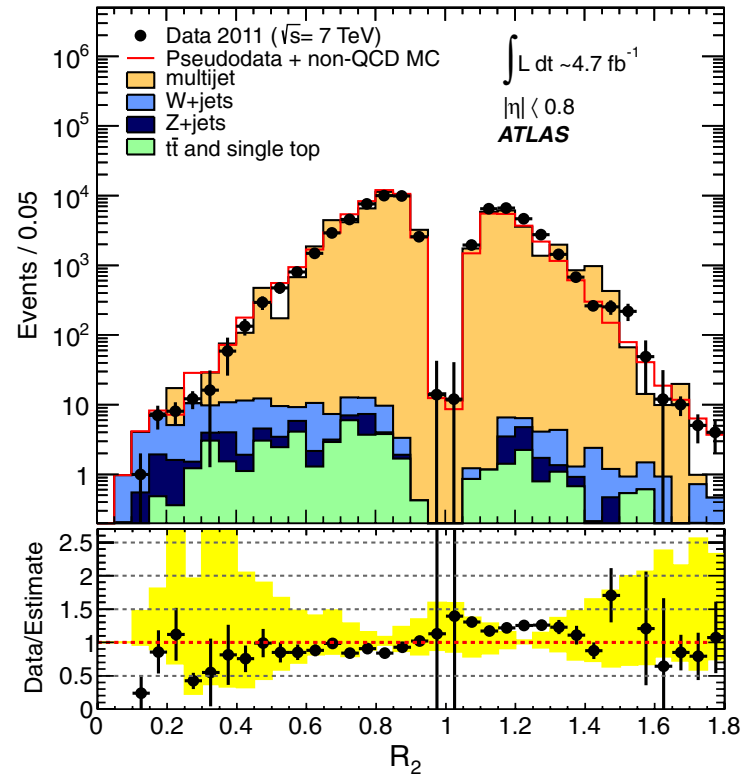

(a)

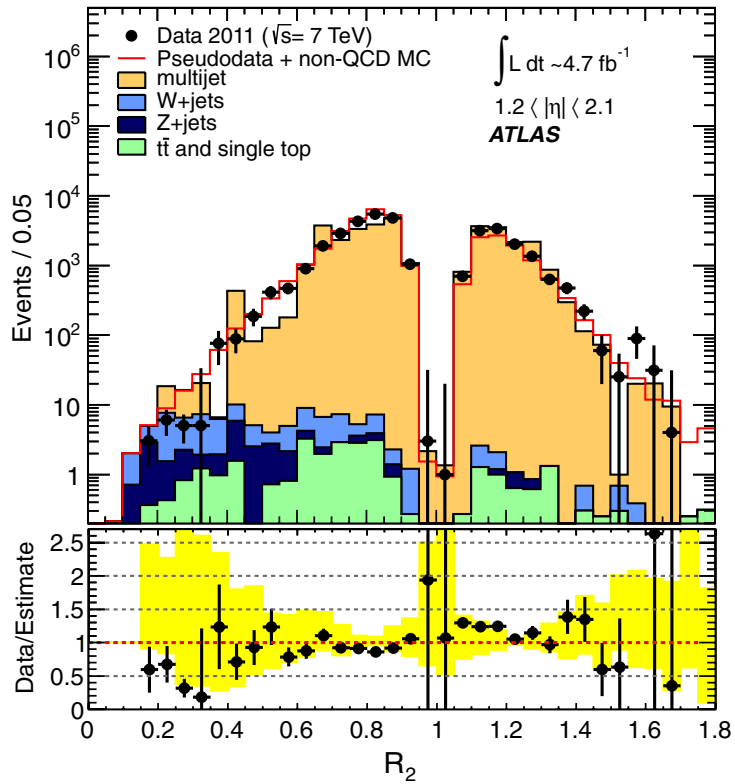

(c) isolated from other truth jets by $\Delta R>0.6$. The response is the ratio of the reconstructed detector-level to generatorlevel jet transverse energy.

A "smeared" event is generated by multiplying each jet four-momentum in a seed event by a random number drawn from the response function. The smeared event $E_{\mathrm{T}}^{\text {miss }}$ is computed using the smeared transverse momenta of the jets. The response function measured using $\mathrm{MC}$

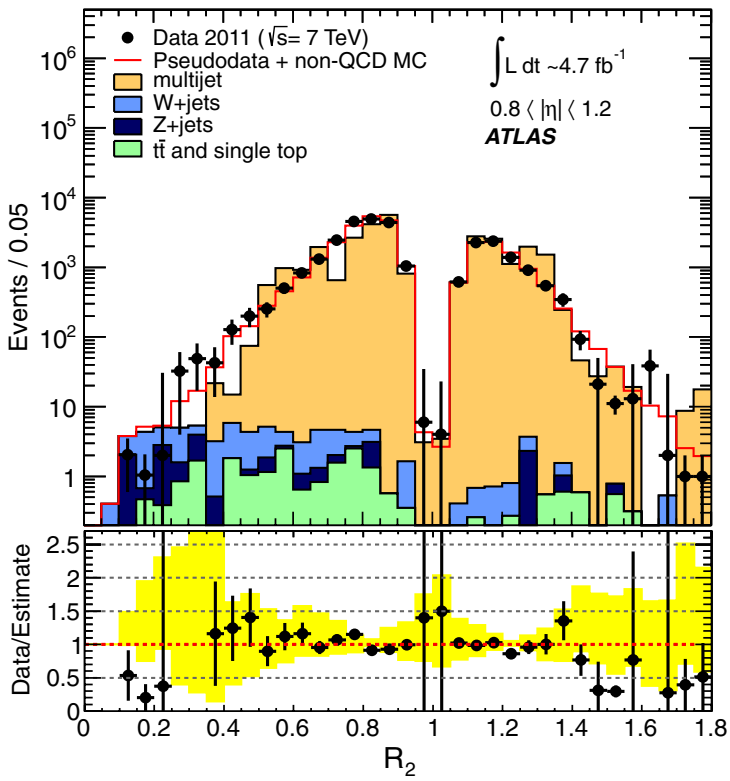

(b)

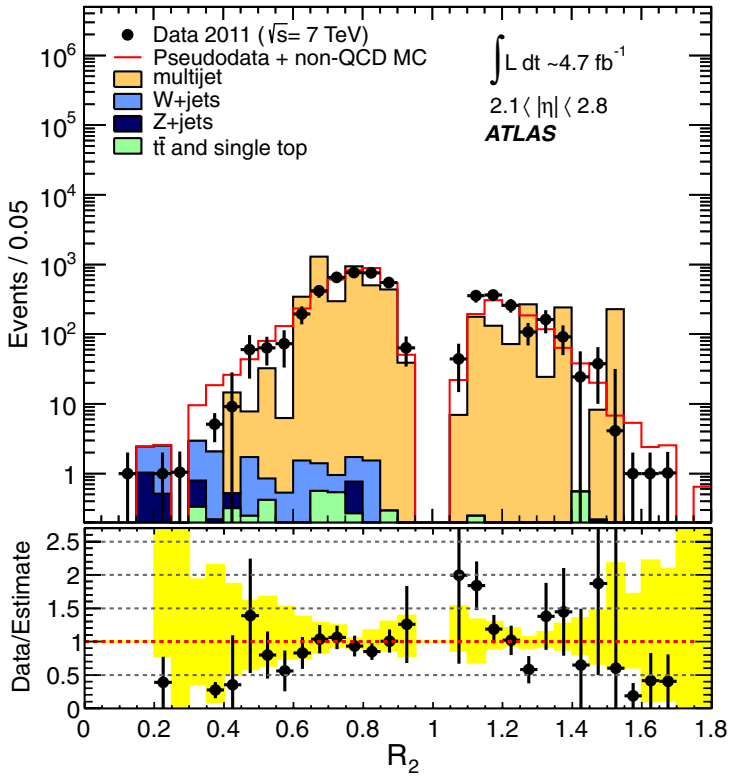

(d)

FIG. 8 (color online). Distributions of $R_{2}$ in four bins (a-d) of $|\eta|$ of the poorly reconstructed jet, for estimated true jet $p_{\mathrm{T}}$, defined as $\left|\vec{p}_{\mathrm{T}}^{J}+\vec{P}_{\mathrm{T}}^{\text {miss }}\right|$, greater than $100 \mathrm{GeV}$. The black points represent collision data while the open medium (red) histogram represents the combined prediction. The jet smearing method described in the text is used to estimate the multijet contribution (referred to in the plots as pseudodata) while MC simulation predictions are used for the other background components. The lower panels show the fractional deviation of the data from the prediction (black points), with the light (yellow) bands showing the multijet uncertainty combined with the MC simulation statistical uncertainty on the nonmultijet estimate. 
simulation is modified using additional Gaussian smearing to widen the jet response, and a correction is applied to the low-side response tail to adjust its shape. These corrections improve the agreement with the data in step (3).

Two dedicated analyses are used to constrain the shape of the jet response function in step (3). The first uses the $p_{\mathrm{T}}$ asymmetry of dijet events. Events with two jets with $|\eta|<$ 2.8 and $p_{\mathrm{T}}>70,50 \mathrm{GeV}$ are selected, where there are no additional jets with $|\eta|<2.8$ and $p_{\mathrm{T}}>40 \mathrm{GeV}$. Events are vetoed if they contain any jet with $p_{\mathrm{T}}>20 \mathrm{GeV}$ and $\eta>2.8$. The $p_{\mathrm{T}}$ asymmetry is given by

$$
A\left(p_{\mathrm{T}, 1}, p_{\mathrm{T}, 2}\right)=\frac{p_{\mathrm{T}, 1}-p_{\mathrm{T}, 2}}{p_{\mathrm{T}, 1}+p_{\mathrm{T}, 2}},
$$

where the indices correspond to the jet $p_{\mathrm{T}}$ ordering. This distribution is sensitive to the Gaussian response of the jets and to any non-Gaussian tails. A fit of pseudodata to the collision-data asymmetry distribution is used to adjust the response function generating the pseudodata.

A second analysis studies the $R_{2}$ distribution of $\geq 3$-jet events where topological selections ensure that one jet is unambiguously associated in $\phi$ with the $E_{\mathrm{T}}^{\text {miss }}$ in the event. The response of the detector to this jet is then given approximately by the quantity $R_{2}$ defined by

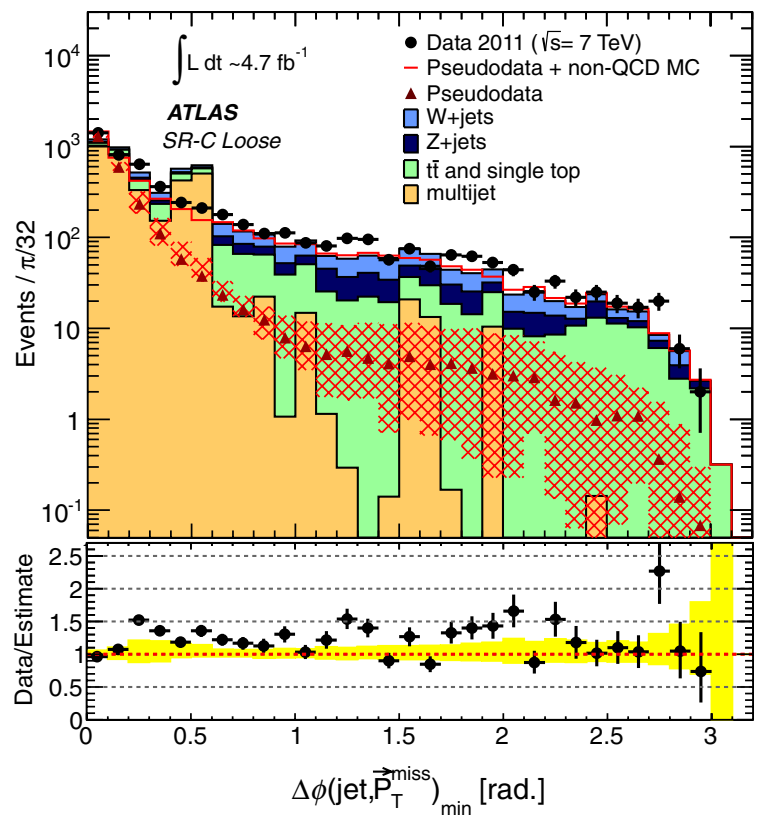

(a)

$$
R_{2} \equiv \frac{\vec{p}_{\mathrm{T}}^{J} \cdot\left(\vec{p}_{\mathrm{T}}^{J}+\vec{P}_{\mathrm{T}}{ }^{\mathrm{miss}}\right)}{\left|\vec{p}_{\mathrm{T}}^{J}+\vec{P}_{\mathrm{T}}{ }^{\text {iss }}\right|^{2}}
$$

where $\vec{p}_{\mathrm{T}}^{J}$ is understood to be the reconstructed $p_{\mathrm{T}}$ of the jet associated with the $E_{\mathrm{T}}^{\mathrm{miss}}$. This distribution is sensitive to the tails of the response function from mismeasured jets. When the $p_{\mathrm{T}}$ of the jet is undermeasured, $\vec{P}_{\mathrm{T}}{ }^{\text {miss }}$ lies parallel to $\vec{p}_{\mathrm{T}}^{J}$ and hence $R_{2}<1$. Conversely, when the $p_{\mathrm{T}}$ of the jet is overmeasured, $\vec{P}_{\mathrm{T}}{ }^{\text {miss }}$ lies antiparallel to $\vec{p}_{\mathrm{T}}^{J}$ and hence $R_{2}>1$. Fits are performed in $p_{\mathrm{T}}$ and $\eta$ bins in order to constrain the parameters describing the low-side response function tail, which affects primarily the region with $R_{2} \ll 1$.

The $R_{2}$ distribution provides a sensitive test of the response function and hence of the background estimate in different regions of the detector, such as the transition between the barrel and end-cap calorimeters, where the energy resolution is degraded by the presence of dead material. The data are divided into four regions according to the $\eta$ of the poorly reconstructed jet associated with the $E_{\mathrm{T}}^{\text {miss }}$, shown in Fig. 8. The estimates agree well, with the data indicating that non-Gaussian fluctuations are not strongly $\eta$ dependent. Given the good agreement observed between the data and estimates, no uncertainty is associated

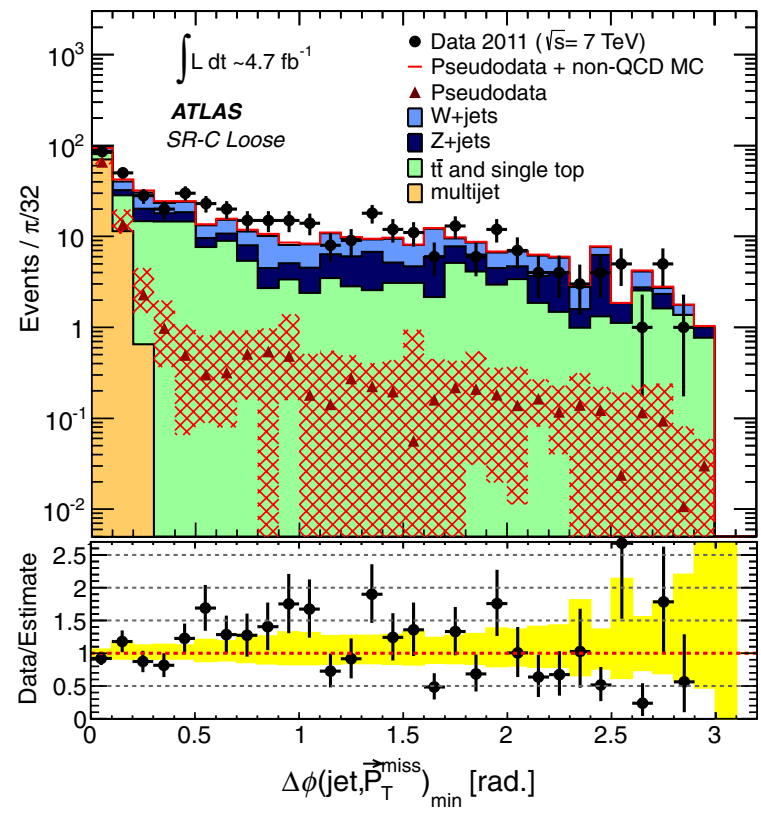

(b)

FIG. 9 (color online). Comparison of observed and predicted distributions of $\Delta \phi\left(\right.$ jet $_{i}, \vec{P}_{\mathrm{T}}$ miss $_{\text {min }}$ for the leading three jets $\left[\Delta \phi\left(\text { jet }_{i}, \vec{P}_{\mathrm{T}}^{\text {miss }}\right)_{\text {min }}(i=\{1,2,3\})\right]$, (a) after all selections except for those on $\Delta \phi\left(\text { jet }_{i}, \vec{P}_{\mathrm{T}}{ }^{\text {miss }}\right)_{\min }, m_{\mathrm{eff}}$ and $E_{\mathrm{T}}^{\text {miss }} / m_{\mathrm{eff}}$ and (b) after all selections except for that on $\Delta \phi\left(\text { jet }_{i}, \vec{P}_{\mathrm{T}}{ }^{\text {miss }}\right)_{\min }$ for signal region $\mathrm{C}$ loose. The histograms show the MC simulation estimates of each background component. The medium (maroon) triangles show the multijet estimates from the jet smearing technique, normalized in the regions with $\Delta \phi\left(\text { jet }_{i}, \vec{P}_{\mathrm{T}}{ }^{\text {miss }}\right)_{\min }(i=\{1,2,3\})<0.2 \mathrm{rad}$, which replaces the multijet MC simulation estimate (denoted with a histogram) in the main analysis. The hatched region denotes the total uncertainty on the multijet estimate including statistical uncertainties from the seed event sample and the smearing procedure, systematic uncertainties in the jet response function, and bias in the seed event selection. The lower panels show the fractional deviation of the data from the prediction (black points), with the light (yellow) bands showing the multijet uncertainty combined with the MC simulation statistical uncertainty on the nonmultijet estimate. 
with the $\eta$ dependence of the response. Following this procedure, a good estimate of the jet response function, including non-Gaussian tails, is obtained.

In order to illustrate the technique, Fig. 9 shows comparisons between SM MC simulation predictions, data and the jet smearing estimate for distributions of $\Delta \phi\left(\text { jet }_{i}, \vec{P}_{\mathrm{T}}^{\text {miss }}\right)_{\min }$ calculated with just the leading three jets. The figure makes use of the earlier stages of the event selections for SR-C loose and its associated multijet control region. The final event selections used in the analysis impose further requirements on $\Delta \phi\left(\text { jet }_{i}, \vec{P}_{\mathrm{T}}^{\text {miss }}\right)_{\min }$ for additional jets with $p_{\mathrm{T}}>40 \mathrm{GeV}$ (see Table I). Good agreement is seen in Fig. 9 both between the data and MC simulation and between the data and the smearing estimate.

In order to check that the above method is robust against changes in pileup conditions, which changed significantly during data taking, the method was repeated with the data divided into subsamples corresponding to four time periods representative of different pileup regimes. No significant dependence upon the level of pileup was found.

The resulting multijet transfer factors between CR2 and $\mathrm{SR}$ for the signal regions are shown in Table V.

\section{E. $W(\rightarrow \ell \nu)+$ jets and $t \bar{t}$ background estimation}

The lepton veto applied to the signal events aims to suppress SM events with an isolated lepton. However, such a veto does not reject all $t \bar{t}$ and $W+$ jets events, particularly when their decay products involve a lepton which is out of acceptance, or not reconstructed, or when the lepton is a hadronically decaying $\tau$.

To estimate the contributions from $W+$ jet and top quark backgrounds in the signal regions, two CRs are defined for each SR, one with a $b$-jet veto (CR3 - enriched in $W+$ jets events) and one with a $b$-tag requirement (CR4-enriched in $t \bar{t}$ events) as defined in Table II. With the exception of the $b$-jet requirement/veto the selections for CR3 and CR4 are identical and hence the two samples are fully anticorrelated. Both of these CRs require exactly one "signal" electron or muon satisfying tighter selection criteria, whose transverse mass, formed with the $E_{\mathrm{T}}^{\mathrm{miss}}$, lies between 30 and $100 \mathrm{GeV}$. The lepton is then modeled as an additional jet, as it would be if it had entered the signal regions. The $\Delta \phi\left(\text { jet }_{i}, \vec{P}_{\mathrm{T}}^{\mathrm{miss}}\right)_{\min }$ and $E_{\mathrm{T}}^{\mathrm{miss}} / m_{\mathrm{eff}}$ criteria which are applied in the corresponding signal regions are not applied to the CRs, in order to increase the CR sample sizes.

In the electron channel, the modeling of the lepton as a jet is physically accurate, as the reconstruction will interpret misidentified electrons in this way. In the muon case, a missed muon will contribute additional missing transverse momentum, rather than an extra jet (although a small fraction of its energy may well be deposited in the calorimeters). When the lepton is a hadronically decaying tau, the behavior lies between these two extremes, with the hadrons being seen as jet activity and the $\tau$ neutrino as missing momentum. In order to be consistent between the electron and muon channels, and to use one high-statistics control region each for top quark and $W$ events, the choice is made to model all leptons as jets. This is justified by the fact that the majority of the background comes from hadronic $\tau$-decay events, for which the behavior of the lepton is more jetlike than $E_{\mathrm{T}}^{\text {miss }}$-like. It should be noted that this choice does not bias the background estimate because identical procedures are applied to data and to MC simulation events used to construct the transfer factors. The procedure has been validated with two alternative choices, in which the lepton is modeled either as missing transverse momentum or as a $\tau$ decay.

The transfer factors are calculated using MC simulation. Several corrections are applied to MC simulation events:

(i) Each event in the CR is weighted by the ratio of the lepton identification efficiency in data to that in simulation. Similarly, the numbers in the signal region are weighted by a corresponding inefficiency scale factor. This weighting is performed on an event-by-event basis, based on the simulated lepton's transverse momentum and pseudorapidity.

(ii) A similar scale factor is applied for the $b$-tagging efficiency (CR4) and fake rate (CR3), which differ between data and simulation [58,59]. This is also performed as an event-by-event weighting.

(iii) The leptons are smeared such that their energy resolution reflects that measured in data.

Various sources of systematic uncertainty on the transfer factors have been considered. For the leptons, the identification efficiency, energy resolution and trigger efficiency are considered. The $b$-tagging efficiency and fake rate, jet energy scale and jet energy resolution (for both $b$-quark and light jets separately), are considered, together with the effect of pileup, of calorimeter electronics failures and of calorimeter energy deposits not associated with physics objects. The fake lepton background is found to be negligible in both CR3 and CR4.

The TFs between CR3, CR4, and the signal regions are given in Table V. Similar TFs are also computed for each channel between CR3, CR4 and the multijet control region $\mathrm{CR} 2$, where $W+$ jets and $t \bar{t}$ events can contribute significantly.

\section{F. Estimated transfer factors}

The transfer factors estimated using the methods described above are summarized in Table $\mathrm{V}$ for each CR. These values, and those between the various CRs, together with the observed event counts in each SR and CR form the inputs to the likelihood fit described in Sec. XI.

\section{SYSTEMATIC UNCERTAINTIES}

Systematic uncertainties arise through the use of the transfer factors relating observations in the control regions 
to background expectations in the signal regions and from the modeling of the SUSY signal. For the transfer factors derived from MC simulation the primary common sources of systematic uncertainty are the JES calibration, jet energy resolution (JER), MC modeling and statistics, and the reconstruction performance in the presence of pileup. The magnitudes of the uncertainties on background estimates arising from these sources are strongly dependent upon the signal region considered. For instance, the uncertainty due to scale variation ranges from $6 \%$ in SR-C loose to $26 \%$ in SR-A tight.

The JES uncertainty has been measured from the complete 2010 data set using the techniques described in Ref. [53] and is around 4\%, with a slight dependence upon $p_{\mathrm{T}}, \eta$ and the proximity to adjacent jets. The JER uncertainty is estimated using the methods discussed in Ref. [53]. Additional contributions are added to both the JES and the JER uncertainties to take account of the effect of pileup at the relatively high luminosity delivered by the LHC in the 2011 run. Both in-time pileup arising from multiple collisions within the same bunch crossing and outof-time pileup, which arises from the detector response to neighboring bunch crossings, are taken into account.

The dominant modeling uncertainty in the MC simulation estimate of the numbers of events in the signal and control regions arises from the impact of QCD jet radiation on $m_{\text {eff }}$. In order to assess this uncertainty, alternative samples were produced with reduced initial parton multiplicities (ALPGEN processes with 0-5 partons rather than $0-6$ partons for $W / Z+$ jets production, and $0-3$ instead of $0-5$ for top quark pair production).

PDF uncertainties are also taken into account. An envelope of cross section predictions is defined using the $68 \%$ confidence level (C.L.) ranges of the CTEQ6.6 [63] (including the $\alpha_{s}$ uncertainty) and MSTW2008 [64] PDF sets, together with independent variations of the factorization and renormalization scales by factors of two or one-half. The nominal cross section value is taken to be the midpoint of the envelope and the uncertainty assigned is half the full width of the envelope, closely following the PDF4LHC recommendations [65].

Additional uncertainties arising from photon and lepton reconstruction efficiency, energy scale and resolution in CR1a, CR1b, CR3 and CR4, b-tag/veto efficiency (CR3 and CR4) and photon acceptance and cosmic ray backgrounds (CR1a) are also considered. Other sources, including the limited number of MC simulation events as well as additional systematic uncertainties related to the response function, are included.

Systematic uncertainties on the expected SUSY signal are estimated through variation of the factorization and renormalization scales between half and twice their default values and by considering the PDF uncertainties. Uncertainties are calculated for individual production processes (e.g. $\tilde{q} \tilde{q}, \tilde{g} \tilde{g}$, etc.).
Initial state radiation (ISR) can significantly affect the signal visibility for SUSY models with small mass splittings. Systematic uncertainties arising from the treatment of ISR are studied by varying the assumed value of $\alpha_{s}$ and the MADGRAPH/PYTHIA6 matching parameters. The uncertainties are found to be negligible for large sparticle masses $(m>300 \mathrm{GeV})$ and mass splittings $(\Delta m>300 \mathrm{GeV})$ and to rise linearly with decreasing mass and decreasing mass splitting to $\sim 30 \%$ for $\Delta m=0$ and $m>300 \mathrm{GeV}$ and to $\sim 40 \%$ for $m=250 \mathrm{GeV}$ and $\Delta m=0$. Signal ISR uncertainties are assumed to be uncorrelated with the corresponding background ISR uncertainties, to ensure a conservative treatment.

\section{RESULTS, INTERPRETATION AND LIMITS}

The numbers of events observed in the data and the numbers of SM events expected to enter the signal regions, determined using the simultaneous likelihood fits (see Secs. III and IX) to the SRs and CRs, are shown in Table III. The use of transfer factors between the CRs and SRs allows systematic uncertainties and nuisance parameters to be dealt with in a coherent way, preserving any correlations, as described above. The free parameters are the background components in each SR, and these are constrained by the CR event counts and the TFs, within their uncertainties. The dominant irreducible background, from $Z+$ jets events, is constrained by both CR1a and CR1b, with CR1a providing the largest statistical weight. The resulting scaled predictions for the background components are shown in Table III. Good agreement is observed between the data and the SM predictions, with no significant excesses found. The fitted predictions for the various background components agree well with the expectations from MC simulation before the fits, once theoretical uncertainties are accounted for.

Data from all the channels are used to set limits on SUSY models, taking the SR with the best expected sensitivity at each point in parameter space. A profile $\log$-likelihood ratio test in combination with the $\mathrm{CL}_{\mathrm{s}}$ prescription [66] is used to derive 95\% C.L. exclusion regions. An interpretation of the results is presented in Fig. 10(a) as a 95\% C.L. exclusion region in the $\left(m_{\tilde{g}}, m_{\tilde{q}}\right)$ plane for a set of simplified SUSY models with $m_{\tilde{\chi}_{1}^{0}}=0$. In these models the gluino mass and the masses of the squarks of the first two generations are set to the values shown in the figure, up to maximum squark and gluino masses of $2 \mathrm{TeV}$. All other supersymmetric particles, including the squarks of the third generation, are decoupled. The results are also interpreted in the $\tan \beta=10, A_{0}=0, \mu>0$ slice of MSUGRA/CMSSM models [67] in Fig. 10(b). In these models, ISASUSY from ISAJET [68] v7.80 is used to calculate the decay tables and to guarantee consistent electroweak symmetry breaking.

In the simplified model with light neutralinos, with the assumption that the colored sparticles are directly 


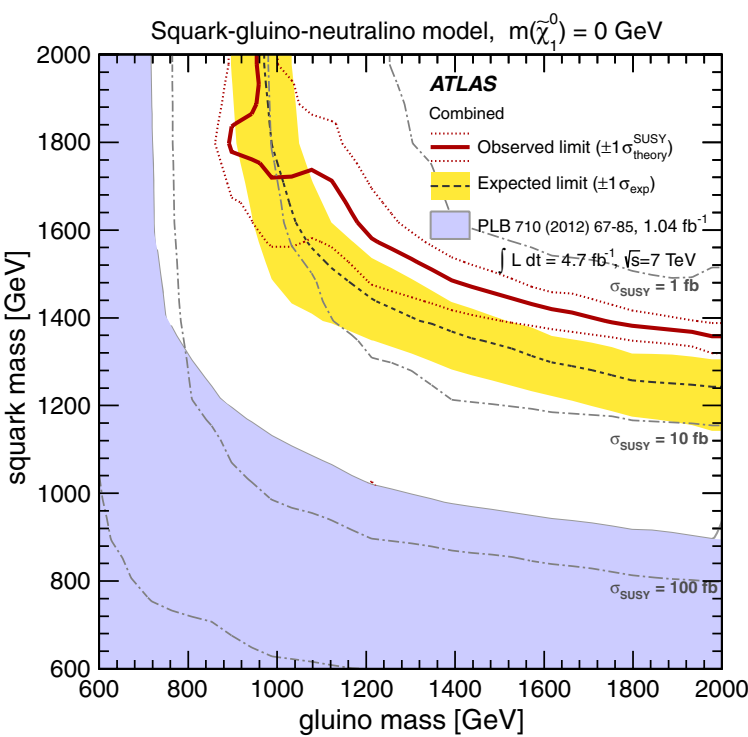

(a)

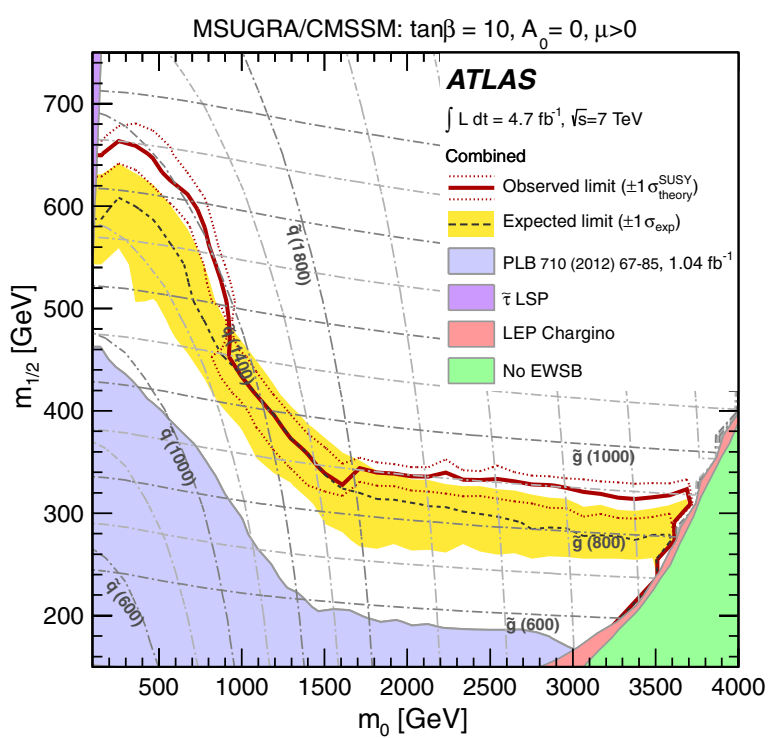

(b)

FIG. 10 (color online). The 95\% $\mathrm{CL}_{\mathrm{s}}$ exclusion limits on (a) the $\left(m_{\tilde{g}}, m_{\tilde{q}}\right)$ plane in a simplified MSSM scenario with only strong production of gluinos and first- and second-generation squarks, with direct decays to jets and neutralinos; (b) the $\left(m_{0}, m_{1 / 2}\right)$ plane of MSUGRA/CMSSM for $\tan \beta=10, A_{0}=0$ and $\mu>0$. Exclusion limits are obtained by using the signal region with the best expected sensitivity at each point. The black dashed lines show the expected limits, with the light (yellow) bands indicating the $1 \sigma$ excursions due to experimental uncertainties. Observed limits are indicated by medium (maroon) curves, where the solid contour represents the nominal limit, and the dotted lines are obtained by varying the cross section by the theoretical scale and PDF uncertainties. Previous results from ATLAS [17] are represented by the shaded region (blue) at bottom left in each case. The region excluded by chargino searches at LEP is taken from Ref. [71].

produced and decay directly to jets and $E_{\mathrm{T}}^{\mathrm{miss}}$, the limit on the gluino mass is approximately $860 \mathrm{GeV}$, and that on the squark mass is $1320 \mathrm{GeV}$. Squarks and gluinos with equal masses below $1410 \mathrm{GeV}$ are excluded. These values are derived from the lower edge of the $1 \sigma$ observed limit band, to take account of the theoretical uncertainties on the SUSY cross sections in a conservative fashion. In the MSUGRA/CMSSM case, the limit on $m_{1 / 2}$ reaches

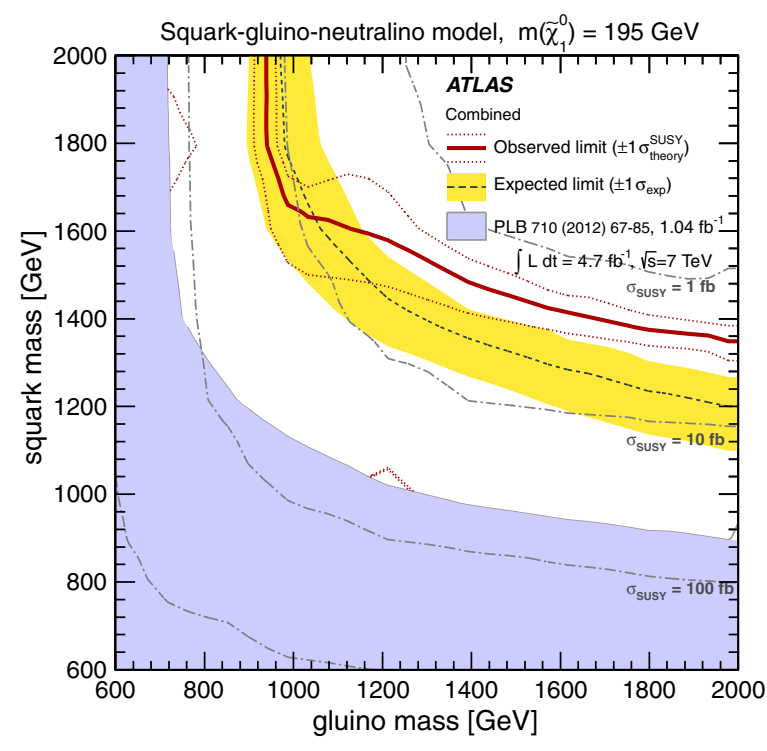

(a)

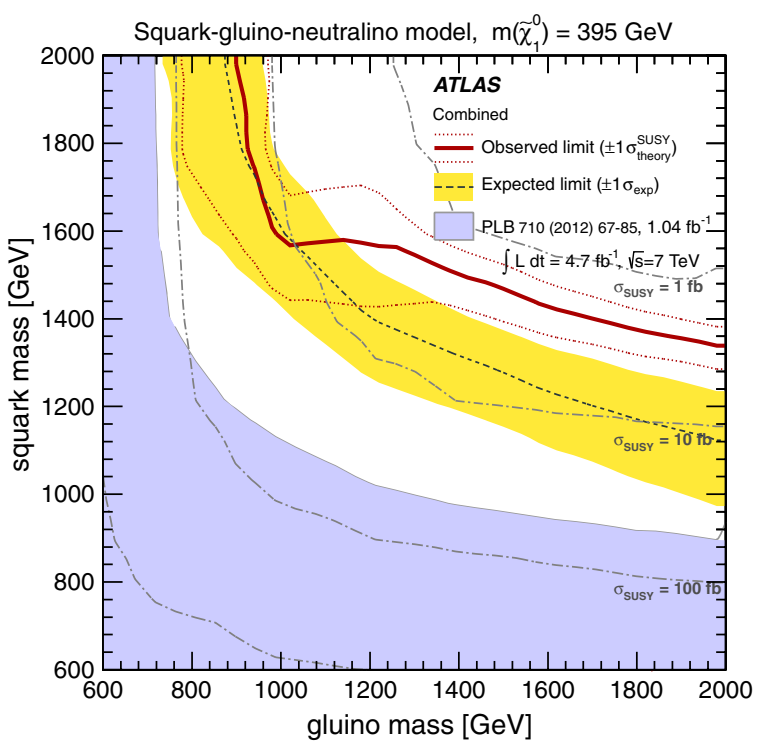

(b)

FIG. 11 (color online). The $95 \% \mathrm{CL}_{\mathrm{s}}$ exclusion limits on the $\left(m_{\tilde{g}}, m_{\tilde{q}}\right)$ plane in MSSM models with nonzero neutralino masses. Combined observed exclusion limits are based on the best expected $\mathrm{CL}_{\mathrm{s}}$ per grid point as for Fig. 10(a), but with an LSP mass of (a) 195 and (b) $395 \mathrm{GeV}$. Curves are as defined in Fig. 10(a). The letters overlaid on the plot show the SR that contributes the best sensitivity at each point. Previous results from ATLAS [17] are represented by the shaded region (blue) at bottom left in each case. 


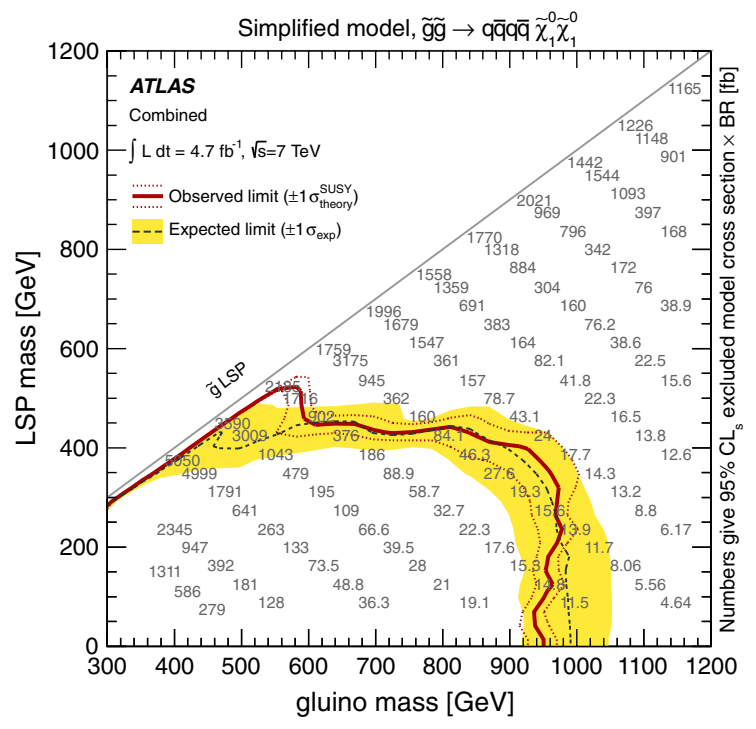

(a)

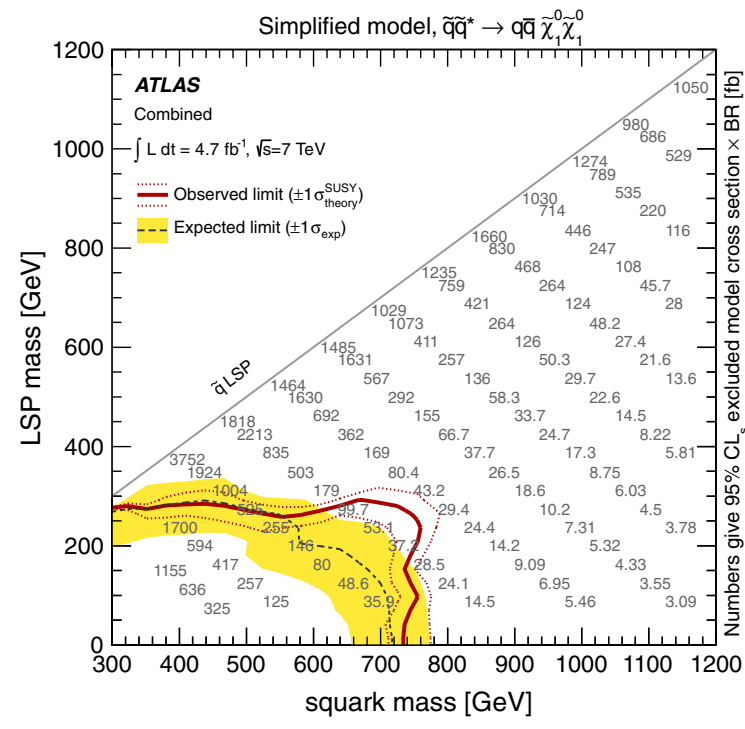

(b)

FIG. 12 (color online). The 95\% $\mathrm{CL}_{\mathrm{s}}$ exclusion limits on simplified models assuming direct production of (a) gluino pairs with decoupled squarks or (b) squark pairs with decoupled gluinos, each decaying to two jets, or one jet, respectively, and a neutralino LSP. 95\% Exclusion limits are obtained by using the signal region with the best expected sensitivity at each point. The black dashed lines show the expected limits, with the light (yellow) bands indicating the $1 \sigma$ excursions due to experimental uncertainties. Observed limits are indicated by medium (maroon) curves, where the solid contour represents the nominal limit, and the dotted lines are obtained by varying the cross section by the theoretical scale and PDF uncertainties. The $95 \% \mathrm{CL}_{\mathrm{s}}$ upper limit on the cross section times branching ratio (in fb) is printed for each model point.

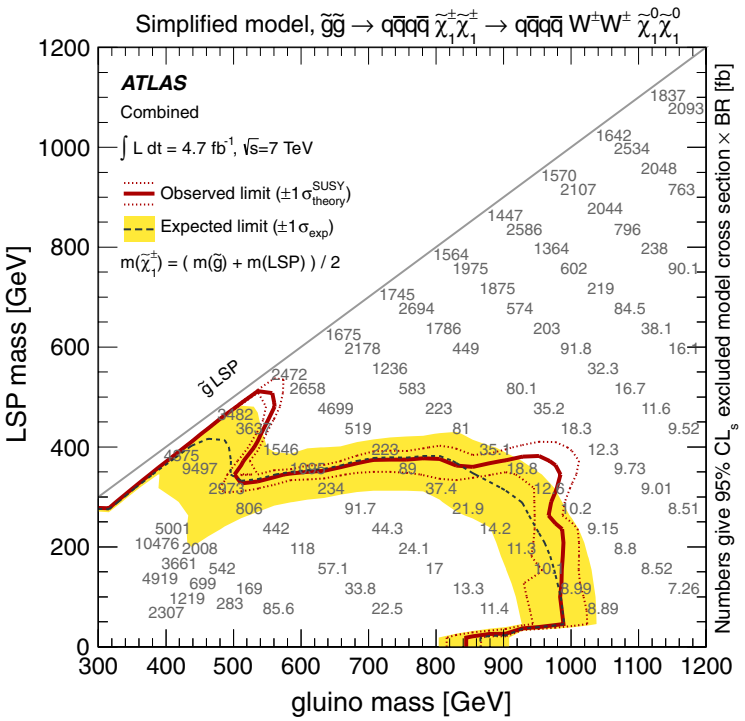

(a)

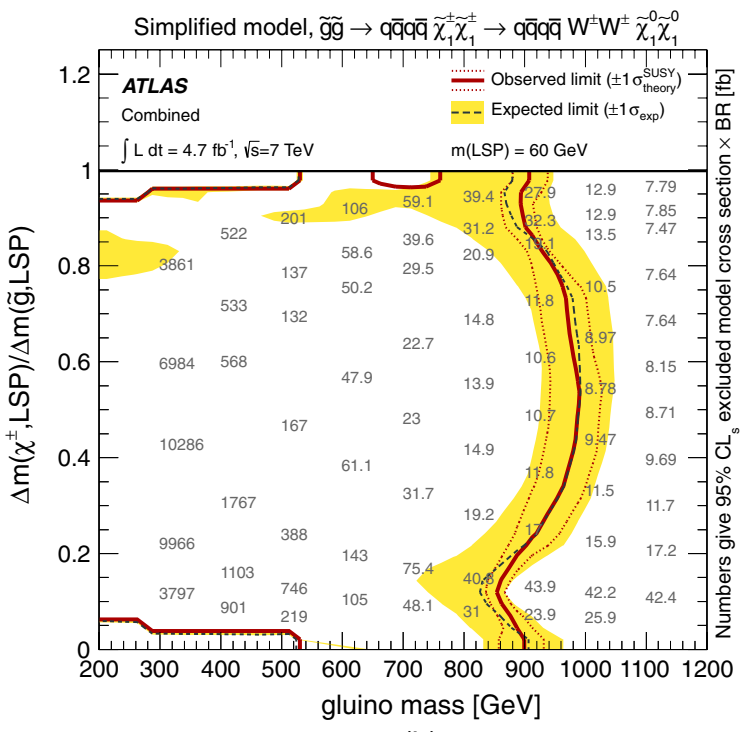

(b)

FIG. 13 (color online). Combined $95 \% \mathrm{CL}_{\mathrm{s}}$ exclusion limits on simplified models assuming direct production of gluino pairs, each decaying via an intermediate chargino to two jets, a $W$ boson and a neutralino LSP. The chargino mass is fixed halfway in between the gluino and LSP masses in figure (a). The neutralino mass is fixed at $60 \mathrm{GeV}$ in figure (b), where the $y$ axis shows the ratio of the chargino-LSP mass splitting to the gluino-LSP mass splitting. The black dashed lines show the expected limits, with the light (yellow) bands indicating the $1 \sigma$ excursions due to experimental uncertainties. Observed limits are indicated by medium (maroon) curves, where the solid contour represents the nominal limit, and the dotted lines are obtained by varying the cross section by the theoretical scale and PDF uncertainties. The $95 \% \mathrm{CL}_{\mathrm{s}}$ upper limit on the cross section times branching ratio (in fb) is printed for each model point. 
$300 \mathrm{GeV}$ at high $m_{0}$ and $640 \mathrm{GeV}$ for low values of $m_{0}$. The inclusion of signal selections sensitive to larger jet multiplicities has improved significantly the ATLAS reach at large $m_{0}$. When their masses are assumed to be equal, squarks and gluinos with masses below $1360 \mathrm{GeV}$ are excluded.

In Figs. 11(a) and 11(b) the limits from Fig. 10(a) are displayed again, but with the LSP mass set to 195 and $395 \mathrm{GeV}$, respectively. For both values, only minor differences are seen in the limit curve, showing that the analysis retains sensitivity for a range of LSP masses. The signal region with the greatest reach is displayed at each point in the plane, showing that the tight, medium and loose selections all contribute to the final result.

In Fig. 12 limits are shown for two cases in which only pair production of (a) gluinos or (b) squarks is kinematically possible, with all other superpartners, except for the neutralino LSP, decoupled. This forces each squark or gluino to decay directly to jets and an LSP, as in the simplified MSSM scenario. Cross sections are evaluated assuming decoupled squarks or gluinos in cases (a) and (b), respectively.

Similar models with only squark or gluino-pair production are shown in Figs. 13 and 14. However, in these variants, the sparticle content is augmented by an additional intermediate chargino with mass between the strongly interacting sparticle and the LSP. This allows for production of additional jets or leptons and enriches the phenomenology. In the squark pair-production case, only left-handed squarks of the first and second generations are considered in order to enhance the branching ratios of decay chains incorporating an intermediate chargino. The cross sections have been reduced by $50 \%$ to take this situation properly into account. Two different parameterizations of the masses are shown. Figures 13(a) and 14(a) vary the squark/gluino mass and the LSP mass, keeping the chargino mass exactly midway between those two. In Figs. 13(b) and 14(b), the LSP mass is instead held fixed, with the ratio of the chargino-LSP mass splitting to the squark/gluino-LSP mass splitting defining the $y$ axis. When either mass splitting is large sensitivity to the model is enhanced by kinematics.

The "compressed SUSY" models suggested in Refs. [69,70] are also considered. In these models, the basic sparticle content and spectrum are similar to that in the CMSSM, but the sizes of all mass splittings are controlled by a compression factor. The squark mass is set to $96 \%$ of the gluino mass. For presentation purposes, the limits are plotted against the gluino mass and the largest mass splitting, i.e. that between gluino and LSP. Exclusion plots are shown in Fig. 15 for three classes of model: one in which all sparticle content is present, a second in which all the neutralinos and charginos apart from the LSP are taken to be sufficiently heavy to decouple, and a third in which the squarks instead are decoupled.

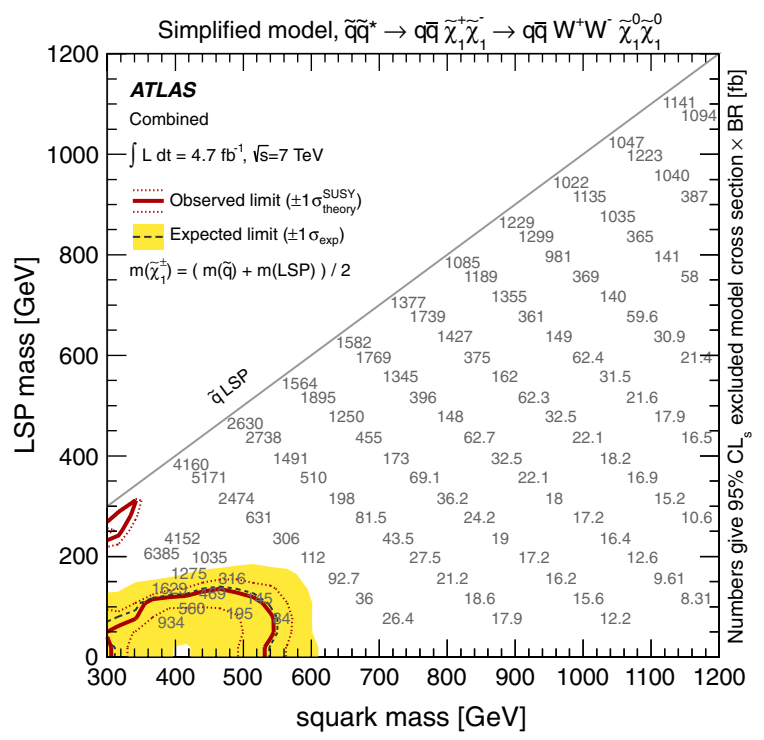

(a)

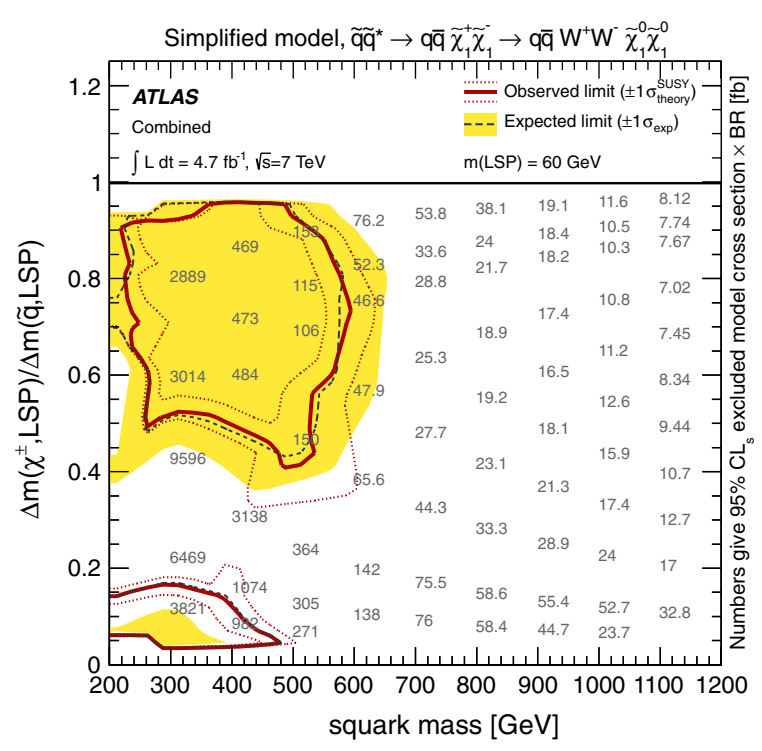

(b)

FIG. 14 (color online). Combined 95\% $\mathrm{CL}_{\mathrm{s}}$ exclusion limits on simplified models assuming direct production of left-handed squarkantisquark pairs, each decaying via an intermediate chargino to two jets, a $W$ boson and a neutralino LSP. The chargino mass is fixed halfway in between the squark and LSP masses in figure (a). In figure (b) the neutralino mass is fixed at $60 \mathrm{GeV}$; the $y$ axis shows the ratio of the chargino-LSP mass splitting to the squark-LSP mass splitting. The black dashed lines show the expected limits, with the light (yellow) bands indicating the $1 \sigma$ excursions due to experimental uncertainties. Observed limits are indicated by medium (maroon) curves, where the solid contour represents the nominal limit, and the dotted lines are obtained by varying the cross section by the theoretical scale and PDF uncertainties. The $95 \% \mathrm{CL}_{\mathrm{s}}$ upper limit on the cross section times branching ratio (in fb) is printed for each model point. 


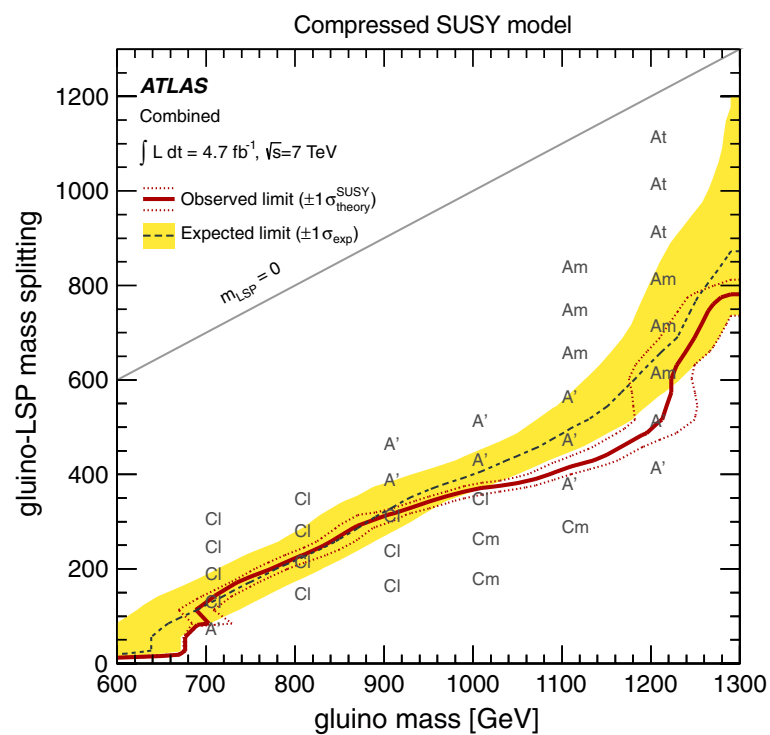

(a)

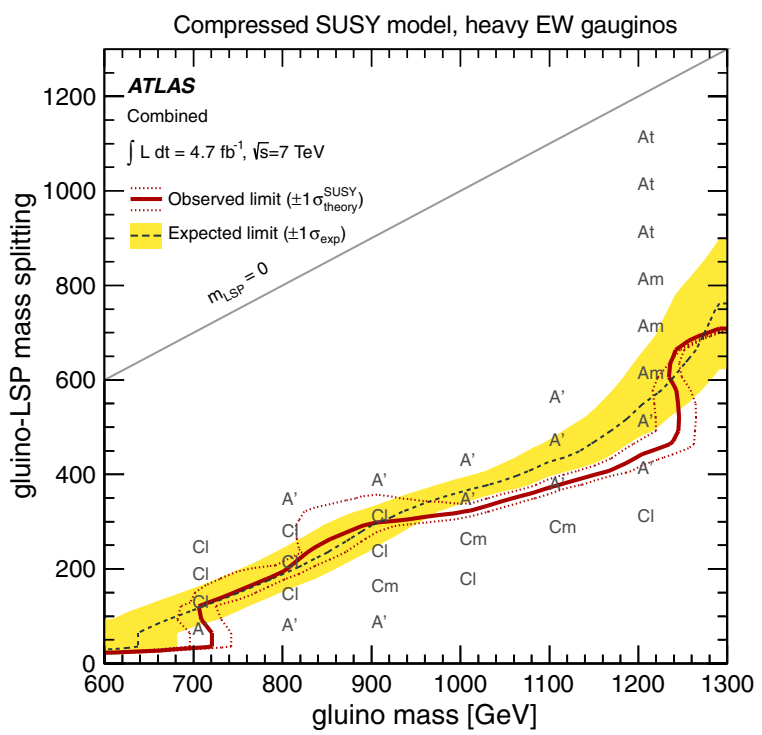

(b)

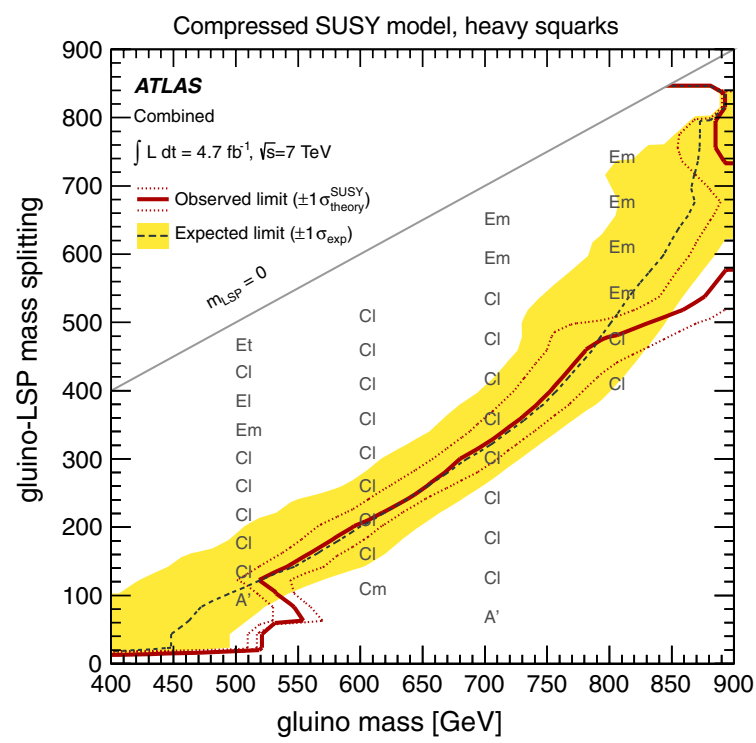

(c)

FIG. 15 (color online). Combined $95 \% \mathrm{CL}_{\mathrm{s}}$ exclusion limits for the compressed SUSY models discussed in the text. In figure (a) all squarks, electroweak gauginos and the gluino are kinematically accessible. In figure (b) neutralinos (apart from the LSP) and charginos are decoupled. In figure (c) squarks are decoupled. The black dashed lines show the expected limits, with the light (yellow) bands indicating the $1 \sigma$ excursions due to experimental uncertainties. Observed limits are indicated by medium (maroon) curves, where the solid contour represents the nominal limit, and the dotted lines are obtained by varying the cross section by the theoretical scale and PDF uncertainties. The letters overlaid on the plot show the SR that contributes the best sensitivity at each point.

\section{SUMMARY}

This paper reports a search for supersymmetry in final states containing high- $p_{\mathrm{T}}$ jets, missing transverse momentum and no electrons with $p_{\mathrm{T}}>20 \mathrm{GeV}$ or muons with $p_{\mathrm{T}}>10 \mathrm{GeV}$. Data recorded by the ATLAS experiment at the LHC at $\sqrt{s}=7 \mathrm{TeV}$ corresponding to an integrated luminosity of $4.7 \mathrm{fb}^{-1}$ have been used. Good agreement is seen between the numbers of events observed in the signal regions and the numbers of events expected from SM sources. The exclusion limits placed on non-SM cross sections impose new constraints on scenarios with novel physics.

The results are interpreted in both a simplified model containing only squarks of the first two generations, a gluino octet and a massless neutralino, as well as in MSUGRA/CMSSM models with $\tan \beta=10, A_{0}=0$ and $\mu>0$. In the simplified model, gluino and squark masses below 860 and $1320 \mathrm{GeV}$, respectively, are excluded at the 95\% confidence level for squark or gluino masses below $2 \mathrm{TeV}$. When assuming their masses to be equal, squarks 
and gluinos with masses below $1410 \mathrm{GeV}$ are excluded. In the MSUGRA/CMSSM case, the limit on $m_{1 / 2}$ reaches $300 \mathrm{GeV}$ at high $m_{0}$ and $640 \mathrm{GeV}$ for low values of $m_{0}$. Squarks and gluinos with equal masses below $1360 \mathrm{GeV}$ are excluded in this scenario. These results are shown to be relatively insensitive to the assumption of a light LSP, up to LSP masses of about $400 \mathrm{GeV}$. Limits are also placed in the parameter space of a SUSY model with a compressed mass spectrum.

\section{ACKNOWLEDGMENTS}

We thank CERN for the very successful operation of the LHC, as well as the support staff from our institutions without whom ATLAS could not be operated efficiently. We acknowledge the support of ANPCyT, Argentina; YerPhI, Armenia; ARC, Australia; BMWF and FWF, Austria; ANAS, Azerbaijan; SSTC, Belarus; CNPq and FAPESP, Brazil; NSERC, NRC and CFI, Canada; CERN; CONICYT, Chile; CAS, MOST and NSFC, China; COLCIENCIAS, Colombia; MSMT CR, MPO CR and VSC CR, Czech Republic; DNRF, DNSRC and Lundbeck Foundation, Denmark; EPLANET and ERC,
European Union; IN2P3-CNRS, CEA-DSM/IRFU, France; GNSF, Georgia; BMBF, DFG, HGF, MPG and AvH Foundation, Germany; GSRT, Greece; ISF, MINERVA, GIF, DIP and Benoziyo Center, Israel; INFN, Italy; MEXT and JSPS, Japan; CNRST, Morocco; FOM and NWO, Netherlands; BRF and RCN, Norway; MNiSW, Poland; GRICES and FCT, Portugal; MERYS (MECTS), Romania; MES of Russia and ROSATOM, Russian Federation; JINR; MSTD, Serbia; MSSR, Slovakia; ARRS and MVZT, Slovenia; DST/NRF, South Africa; MICINN, Spain; SRC and Wallenberg Foundation, Sweden; SER, SNSF and Cantons of Bern and Geneva, Switzerland; NSC, Taiwan; TAEK, Turkey; STFC, the Royal Society and Leverhulme Trust, United Kingdom; DOE and NSF, United States of America. The crucial computing support from all WLCG partners is acknowledged gratefully, in particular, from CERN and the ATLAS Tier-1 facilities at TRIUMF (Canada), NDGF (Denmark, Norway, Sweden), CC-IN2P3 (France), KIT/GridKA (Germany), INFN-CNAF (Italy), NL-T1 (Netherlands), PIC (Spain), ASGC (Taiwan), RAL (United Kingdom) and BNL (USA) and in the Tier-2 facilities worldwide.
[1] L. Evans and P. Bryant, JINST 3, S08001 (2008).

[2] H. Miyazawa, Prog. Theor. Phys. 36, 1266 (1966).

[3] P. Ramond, Phys. Rev. D 3, 2415 (1971).

[4] Y. A. Golfand and E. P. Likhtman, Pis'ma Zh. Eksp. Teor. Fiz. 13, 452 (1971) [JETP Lett. 13, 323 (1971)].

[5] A. Neveu and J. H. Schwarz, Nucl. Phys. B31, 86 (1971).

[6] A. Neveu and J. H. Schwarz, Phys. Rev. D 4, 1109 (1971).

[7] J. Gervais and B. Sakita, Nucl. Phys. B34, 632 (1971).

[8] D. V. Volkov and V.P. Akulov, Phys. Lett. 46B, 109 (1973).

[9] J. Wess and B. Zumino, Phys. Lett. 49B, 52 (1974).

[10] J. Wess and B. Zumino, Nucl. Phys. B70, 39 (1974).

[11] P. Fayet, Phys. Lett. 64B, 159 (1976).

[12] P. Fayet, Phys. Lett. 69B, 489 (1977).

[13] G. R. Farrar and P. Fayet, Phys. Lett. 76B, 575 (1978).

[14] P. Fayet, Phys. Lett. 84B, 416 (1979).

[15] S. Dimopoulos and H. Georgi, Nucl. Phys. B193, 150 (1981).

[16] ATLAS Collaboration, Phys. Rev. D 85, 012006 (2012).

[17] ATLAS Collaboration, Phys. Lett. B 710, 67 (2012).

[18] ATLAS Collaboration, JINST 3, S08003 (2008).

[19] ATLAS uses a right-handed coordinate system with its origin at the nominal interaction point in the center of the detector and the $z$ axis along the beam pipe. Cylindrical coordinates $(r, \phi)$ are used in the transverse plane, $\phi$ being the azimuthal angle around the beam pipe. The pseudorapidity $\eta$ is defined in terms of the polar angle $\theta$ by $\eta=-\ln \tan (\theta / 2)$. The transverse momentum $p_{\mathrm{T}}$ is defined in terms of the magnitude of the three momentum $|p|$ by $p_{\mathrm{T}}=|p| \sin \theta$.
[20] ATLAS Collaboration, Eur. Phys. J. C 71, 1630 (2011).

[21] ATLAS Collaboration, Report No. ATLAS-CONF-2011116, http://cdsweb.cern.ch/record/1376384.

[22] T. Sjostrand, S. Mrenna, and P.Z. Skands, J. High Energy Phys. 05 (2006) 026.

[23] A. Sherstnev and R. S. Thorne, Eur. Phys. J. C 55, 553 (2008).

[24] M. L. Mangano, M. Moretti, F. Piccinini, R. Pittau, and A. D. Polosa, J. High Energy Phys. 07 (2003) 001.

[25] J. Pumplin, D. R. Stump, J. Huston, H. L. Lai, P. M. Nadolsky, and W.-K. Tung, J. High Energy Phys. 07 (2002) 012.

[26] T. Gleisberg, S. Höche, F. Krauss, M. Schönherr, S. Schumann, F. Siegert, and J. Winter, J. High Energy Phys. 02 (2009) 007.

[27] B. P. Kersevan and E. Richter-Was, arXiv:hep-ph/0405247.

[28] G. Corcella, I. G. Knowles, G. Marchesini, S. Moretti, K. Odagiri, P. Richardson, M. H. Seymour, and B. R. Webber, J. High Energy Phys. 01 (2001) 010.

[29] G. Corcella, I. G. Knowles, G. Marchesini, S. Moretti, K. Odagiri, P. Richardson, M. H. Seymour, and B. R. Webber, arXiv:hep-ph/0210213.

[30] J.M. Butterworth, J. R. Forshaw, and M. H. Seymour, Z. Phys. C 72, 637 (1996).

[31] M. Bahr et al., Eur. Phys. J. C 58, 639 (2008).

[32] J. Alwall, P. Demin, S. de Visscher, R. Frederix, M. Herquet, F. Maltoni, T. Plehn, D. L. Rainwater, and T. Stelzer, J. High Energy Phys. 09 (2007) 028.

[33] F. Maltoni and T. Stelzer, J. High Energy Phys. 02 (2003) 027. 
[34] W. Beenakker, R. Hopker, M. Spira, and P. M. Zerwas, Nucl. Phys. B492, 51 (1997).

[35] A. Kulesza and L. Motyka, Phys. Rev. Lett. 102, 111802 (2009).

[36] A. Kulesza and L. Motyka, Phys. Rev. D 80, 095004 (2009).

[37] W. Beenakker, S. Brensing, M. Krämer, A. Kulesza, E. Laenen, and I. Niessen, J. High Energy Phys. 12 (2009) 041.

[38] W. Beenakker, S. Brensing, M. Krämer, A. Kulesza, E. Laenen, L. Motyka, and I. Niessen, Int. J. Mod. Phys. A 26, 2637 (2011).

[39] The NLL correction is used for squark and gluino production when the squark and gluino masses lie between $200 \mathrm{GeV}$ and $2 \mathrm{TeV}$. Following the convention used in the NLO calculators the squark mass is defined as the average of the squark masses in the first two generations. In the case of gluino-pair (associated squark-gluino) production processes, the NLL calculations were extended up to squark masses of $4.5 \mathrm{TeV}(3.5 \mathrm{TeV})$. For masses outside this range and for other types of production processes (i.e. electroweak and associated strong and electroweak) cross sections at NLO accuracy obtained with PROSPINO [34] are used.

[40] M. Krämer, A. Kulesza, R. van der Leeuw, M. Mangano, S. Padhi, T. Plehn, and X. Portell, arXiv:1206.2892.

[41] ATLAS Collaboration, Report No. ATL-PHYS-PUB2011-009, http://cdsweb.cern.ch/record/1363300.

[42] ATLAS Collaboration, Report No. ATL-PHYS-PUB2011-014, http://cdsweb.cern.ch/record/1400677.

[43] ATLAS Collaboration, Report No. ATL-PHYS-PUB2010-014, http://cdsweb.cern.ch/record/1303025.

[44] ATLAS Collaboration, Eur. Phys. J. C 70, 823 (2010).

[45] S. Agostinelli et al. (GEANT4 Collaboration), Nucl. Instrum. Methods Phys. Res., Sect. A 506, 250 (2003).

[46] ATLAS Collaboration, Eur. Phys. J. C 72, 1849 (2012).

[47] ATLAS Collaboration, Report No. ATLAS-CONF-2011072, http://cdsweb.cern.ch/record/1351836.

[48] The electromagnetic energy scale is the basic calorimeter signal scale for the ATLAS calorimeters. It has been established using test-beam measurements for electrons and muons to give the correct response for the energy deposited in electromagnetic showers, while it does not correct for the lower response of the calorimeter to hadrons

[49] For jets, the amount of transverse energy $\left(E_{T}\right)$ lost in the dead region can be estimated from the energy depositions in the neighboring calorimeter cells. If this lost $E_{T}$ projected along the $\vec{P}_{\mathrm{T}}$ miss direction amounts to more than $10 \mathrm{GeV}$ and constitutes more than $10 \%$ of the $E_{\mathrm{T}}^{\mathrm{miss}}$, the event is rejected.

[50] M. Cacciari, G. P. Salam, and G. Soyez, J. High Energy Phys. 04 (2008) 063.

[51] M. Cacciari and G. P. Salam, Phys. Lett. B 641, 57 (2006).

[52] W. Lampl et al., Report No. ATL-LARG-PUB-2008-002, http://cdsweb.cern.ch/record/1099735.

[53] ATLAS Collaboration, arXiv:1112.6426 [Eur. Phys. J. C (to be published)].

[54] ATLAS Collaboration, Eur. Phys. J. C 72, 1909 (2012).

[55] ATLAS Collaboration, Report No. ATLAS-CONF-2011046, http://cdsweb.cern.ch/record/1338575.

[56] ATLAS Collaboration, Report No. ATLAS-CONF-2011063, http://cdsweb.cern.ch/record/1345743.

[57] ATLAS Collaboration, Phys. Rev. D 85, 092014 (2012).

[58] ATLAS Collaboration, Report No. ATLAS-CONF-2011102, http://cdsweb.cern.ch/record/1369219.

[59] ATLAS Collaboration, Report No. ATLAS-CONF-2012043 http://cdsweb.cern.ch/record/1435197.

[60] S. Ask, M. Parker, T. Sandoval, M. Shea, and W. Stirling, J. High Energy Phys. 10 (2011) 058.

[61] T. Sjostrand, S. Mrenna, and P.Z. Skands, Comput. Phys. Commun. 178, 852 (2008).

[62] F. Berends, H. Kuijf, B. Tausk, and W. Giele, Nucl. Phys. B357, 32 (1991).

[63] P. Nadolsky, H.-L. Lai, Q.-H. Cao, J. Huston, J. Pumplin, D. Stump, W.-K. Tung, and C.-P. Yuan, Phys. Rev. D 78, 013004 (2008).

[64] A. Martin, W. Stirling, R. Thorne, and G. Watt, Eur. Phys. J. C 63, 189 (2009).

[65] M. Botje et al., arXiv:1101.0538.

[66] A. Read, J. Phys. G 28, 2693 (2002).

[67] Five parameters are needed to specify a particular MSUGRA/CMSSM model: the universal scalar mass $m_{0}$, the universal gaugino mass $m_{1 / 2}$, the universal trilinear scalar coupling $A_{0}$, the ratio of the vacuum expectation values of the two Higgs fields, $\tan \beta$, and the sign of the Higgsino mass parameter, $\mu= \pm$.

[68] F.E. Paige, S. D. Protopopescu, H. Baer, and X. Tata, arXiv:hep-ph/0312045.

[69] T. J. LeCompte and S. P. Martin, Phys. Rev. D 84, 015004 (2011).

[70] T. J. LeCompte and S. P. Martin, Phys. Rev. D 85, 035023 (2012).

[71] J. Abdallah et al., Eur. Phys. J. C 31, 421 (2003).

G. Aad, ${ }^{47}$ T. Abajyan,${ }^{20}$ B. Abbott, ${ }^{110}$ J. Abdallah, ${ }^{11}$ S. Abdel Khalek, ${ }^{114}$ A. A. Abdelalim, ${ }^{48}$ O. Abdinov, ${ }^{10}$ R. Aben, ${ }^{104}$ B. Abi, ${ }^{111}$ M. Abolins, ${ }^{87}$ O. S. AbouZeid ${ }^{157}$ H. Abramowicz, ${ }^{152}$ H. Abreu, ${ }^{135}$ E. Acerbi, ${ }^{88 a}, 88 b$ B. S. Acharya, ${ }^{163 a, 163 b}$ L. Adamczyk,${ }^{37}$ D. L. Adams, ${ }^{24}$ T. N. Addy,${ }^{55}$ J. Adelman, ${ }^{175}$ S. Adomeit,${ }^{97}$ P. Adragna, ${ }^{74}$ T. Adye, ${ }^{128}$ S. Aefsky, ${ }^{22}$ J. A. Aguilar-Saavedra, ${ }^{123 b, b}$ M. Agustoni, ${ }^{16}$ M. Aharrouche ${ }^{80}$ S. P. Ahlen, ${ }^{21}$ F. Ahles, ${ }^{47}$ A. Ahmad, ${ }^{147}$ M. Ahsan,${ }^{40}$ G. Aielli, ${ }^{132 a, 132 b}$ T. Akdogan, ${ }^{18 a}$ T. P. A. Åkesson, ${ }^{78}$ G. Akimoto, ${ }^{154}$ A. V. Akimov, ${ }^{93}$ M. S. Alam, ${ }^{1}$ M. A. Alam,${ }^{75}$ J. Albert, ${ }^{168}$ S. Albrand,${ }^{54}$ M. Aleksa, ${ }^{29}$ I. N. Aleksandrov, ${ }^{63}$ F. Alessandria,${ }^{88 a}$ C. Alexa, ${ }^{25 \mathrm{a}} \mathrm{G}$. Alexander, ${ }^{152} \mathrm{G}$. Alexandre, ${ }^{48}$ T. Alexopoulos, ${ }^{9}$ M. Alhroob, ${ }^{163 a, 163 \mathrm{c}}$ M. Aliev, ${ }^{15} \mathrm{G}$. Alimonti, ${ }^{88 \mathrm{a}}$ J. Alison, ${ }^{119}$ B. M. M. Allbrooke, ${ }^{17}$ P. P. Allport, ${ }^{72}$ S. E. Allwood-Spiers,${ }^{52}$ J. Almond, ${ }^{81}$ A. Aloisio,,${ }^{101 a, 101 b}$ R. Alon, ${ }^{171}$ A. Alonso, ${ }^{78}$ F. Alonso, ${ }^{69}$ B. Alvarez Gonzalez ${ }^{87}$ M. G. Alviggi, ${ }^{101 a, 101 b}$ K. Amako, ${ }^{64}$ C. Amelung, ${ }^{22}$ 
V. V. Ammosov, ${ }^{127, a}$ A. Amorim, ${ }^{123 a, c}$ N. Amram, ${ }^{152}$ C. Anastopoulos, ${ }^{29}$ L. S. Ancu, ${ }^{16}$ N. Andari, ${ }^{114}$ T. Andeen, ${ }^{34}$

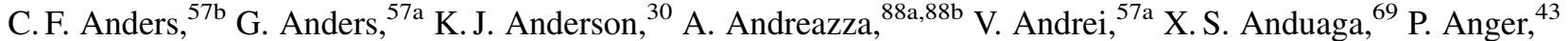

A. Angerami, ${ }^{34}$ F. Anghinolfi, ${ }^{29}$ A. Anisenkov, ${ }^{106}$ N. Anjos, ${ }^{123 a}$ A. Annovi, ${ }^{46}$ A. Antonaki, ${ }^{8}$ M. Antonelli, ${ }^{46}$ A. Antonov, ${ }^{95}$ J. Antos, ${ }^{143 b}$ F. Anulli, ${ }^{131 \mathrm{a}}$ M. Aoki,${ }^{100}$ S. Aoun, ${ }^{82}$ L. Aperio Bella, ${ }^{4}$ R. Apolle, ${ }^{117, d}$ G. Arabidze, ${ }^{87}$ I. Aracena, ${ }^{142}$ Y. Arai, ${ }^{64}$ A. T. H. Arce, ${ }^{44}$ S. Arfaoui, ${ }^{147}$ J-F. Arguin,,${ }^{14}$ E. Arik, ${ }^{18 a, a}$ M. Arik, ${ }^{18 a}$ A. J. Armbruster,${ }^{86}$ O. Arnaez ${ }^{80}$ V. Arnal, ${ }^{79}$ C. Arnault, ${ }^{14}$ A. Artamonov, ${ }^{94}$ G. Artoni,,${ }^{131 a, 131 b}$ D. Arutinov, ${ }^{20}$ S. Asai, ${ }^{154}$

R. Asfandiyarov, ${ }^{172}$ S. Ask, ${ }^{27}$ B. Åsman, ${ }^{145 a, 145 b}$ L. Asquith, ${ }^{5}$ K. Assamagan, ${ }^{24}$ A. Astbury, ${ }^{168}$ M. Atkinson, ${ }^{164}$ B. Aubert, ${ }^{4}$ E. Auge,${ }^{114}$ K. Augsten, ${ }^{126}$ M. Aurousseau, ${ }^{144 a}$ G. Avolio, ${ }^{162}$ R. Avramidou, ${ }^{9}$ D. Axen, ${ }^{167}$ G. Azuelos, ${ }^{92, e}$ Y. Azuma, ${ }^{154}$ M. A. Baak,${ }^{29}$ G. Baccaglioni, ${ }^{88 a}$ C. Bacci, ${ }^{133 a, 133 b}$ A. M. Bach,${ }^{14}$ H. Bachacou, ${ }^{135} \mathrm{~K}$. Bachas, ${ }^{29}$ M. Backes,${ }^{48}$ M. Backhaus, ${ }^{20}$ E. Badescu, ${ }^{25 a}$ P. Bagnaia, ${ }^{131 a, 131 b}$ S. Bahinipati, ${ }^{2}$ Y. Bai,${ }^{32 a}$ D. C. Bailey, ${ }^{157}$ T. Bain, ${ }^{157}$ J. T. Baines, ${ }^{128}$ O. K. Baker, ${ }^{175}$ M. D. Baker ${ }^{24}$ S. Baker,${ }^{76}$ E. Banas, ${ }^{38}$ P. Banerjee, ${ }^{92}$ Sw. Banerjee, ${ }^{172}$ D. Banfi, ${ }^{29}$

A. Bangert, ${ }^{149}$ V. Bansal, ${ }^{168}$ H. S. Bansil,${ }^{17}$ L. Barak,${ }^{171}$ S. P. Baranov, ${ }^{93}$ A. Barbaro Galtieri, ${ }^{14}$ T. Barber, ${ }^{47}$ E. L. Barberio, ${ }^{85}$ D. Barberis, ${ }^{49 a, 49 b}$ M. Barbero, ${ }^{20}$ D. Y. Bardin, ${ }^{63}$ T. Barillari, ${ }^{98}$ M. Barisonzi,${ }^{174}$ T. Barklow, ${ }^{142}$ N. Barlow, ${ }^{27}$ B. M. Barnett, ${ }^{128}$ R. M. Barnett, ${ }^{14}$ A. Baroncelli, ${ }^{133 a}$ G. Barone, ${ }^{48}$ A. J. Barr, ${ }^{117}$ F. Barreiro, ${ }^{79}$ J. Barreiro Guimarães da Costa, ${ }^{56}$ P. Barrillon, ${ }^{114}$ R. Bartoldus, ${ }^{142}$ A. E. Barton, ${ }^{70}$ V. Bartsch, ${ }^{148}$ A. Basye, ${ }^{164}$ R. L. Bates, ${ }^{52}$ L. Batkova, ${ }^{143 a}$ J. R. Batley, ${ }^{27}$ A. Battaglia, ${ }^{16}$ M. Battistin, ${ }^{29}$ F. Bauer, ${ }^{135}$ H. S. Bawa, ${ }^{142, f}$ S. Beale, ${ }^{97}$

T. Beau,${ }^{77}$ P. H. Beauchemin, ${ }^{160}$ R. Beccherle,${ }^{49 a}$ P. Bechtle, ${ }^{20}$ H. P. Beck,${ }^{16}$ A. K. Becker, ${ }^{174}$ S. Becker,${ }^{97}$

M. Beckingham, ${ }^{137}$ K. H. Becks, ${ }^{174}$ A. J. Beddall, ${ }^{18 \mathrm{c}}$ A. Beddall, ${ }^{18 \mathrm{c}}$ S. Bedikian, ${ }^{175}$ V. A. Bednyakov, ${ }^{63}$ C. P. Bee, ${ }^{82}$ L. J. Beemster, ${ }^{104}$ M. Begel ${ }^{24}$ S. Behar Harpaz, ${ }^{151}$ M. Beimforde, ${ }^{98}$ C. Belanger-Champagne, ${ }^{84}$ P. J. Bell ${ }^{48}$ W. H. Bell, ${ }^{48}$ G. Bella, ${ }^{152}$ L. Bellagamba, ${ }^{19 a}$ F. Bellina, ${ }^{29}$ M. Bellomo,${ }^{29}$ A. Belloni,${ }^{56}$ O. Beloborodova, ${ }^{106, g}$

K. Belotskiy, ${ }^{95}$ O. Beltramello, ${ }^{29}$ O. Benary,${ }^{152}$ D. Benchekroun, ${ }^{134 \mathrm{a}} \mathrm{K}$. Bendtz, ${ }^{145 \mathrm{a}, 145 \mathrm{~b}}$ N. Benekos, ${ }^{164}$ Y. Benhammou ${ }^{152}$ E. Benhar Noccioli, ${ }^{48}$ J. A. Benitez Garcia, ${ }^{158 b}$ D. P. Benjamin, ${ }^{44}$ M. Benoit, ${ }^{114}$ J. R. Bensinger, ${ }^{22}$ K. Benslama, ${ }^{129}$ S. Bentvelsen, ${ }^{104}$ D. Berge,${ }^{29}$ E. Bergeaas Kuutmann, ${ }^{41}$ N. Berger, ${ }^{4}$ F. Berghaus, ${ }^{168}$ E. Berglund,${ }^{104}$ J. Beringer, ${ }^{14}$ P. Bernat, ${ }^{76}$ R. Bernhard, ${ }^{47}$ C. Bernius, ${ }^{24}$ T. Berry, ${ }^{75}$ C. Bertella, ${ }^{82}$ A. Bertin,,${ }^{19,19 b}$ F. Bertolucci, ${ }^{121 \mathrm{a}, 121 \mathrm{~b}}$ M. I. Besana, ${ }^{88 a, 88 \mathrm{~b}}$ G. J. Besjes, ${ }^{103}$ N. Besson, ${ }^{135}$ S. Bethke, ${ }^{98}$ W. Bhimji, ${ }^{45}$ R. M. Bianchi, ${ }^{29}$ M. Bianco, ${ }^{71 a, 71 b}$ O. Biebel, ${ }^{97}$ S. P. Bieniek, ${ }^{76}$ K. Bierwagen,${ }^{53}$ J. Biesiada,,${ }^{14}$ M. Biglietti, ${ }^{133 a}$ H. Bilokon, ${ }^{46}$ M. Bindi, ${ }^{19 \mathrm{a}, 19 \mathrm{~b}}$ S. Binet ${ }^{114}$ A. Bingul, ${ }^{18 \mathrm{c}}$ C. Bini, ${ }^{131 \mathrm{a}, 131 \mathrm{~b}}$ C. Biscarat,${ }^{177}$ B. Bittner, ${ }^{98}$ K. M. Black, ${ }^{21}$ R. E. Blair, ${ }^{5}$ J.-B. Blanchard, ${ }^{135}$ G. Blanchot, ${ }^{29}$ T. Blazek, ${ }^{143 a}$ C. Blocker, ${ }^{22}$ J. Blocki, ${ }^{38}$ A. Blondel,${ }^{48}$ W. Blum,${ }^{80}$

U. Blumenschein, ${ }^{53}$ G. J. Bobbink, ${ }^{104}$ V. B. Bobrovnikov, ${ }^{106}$ S. S. Bocchetta ${ }^{78}$ A. Bocci, ${ }^{44}$ C. R. Boddy,${ }^{117}$ M. Boehler ${ }^{47}$ J. Boek, ${ }^{174}$ N. Boelaert, ${ }^{35}$ J. A. Bogaerts, ${ }^{29}$ A. Bogdanchikov, ${ }^{106}$ A. Bogouch,${ }^{89, a}$ C. Bohm, ${ }^{145 a}$ J. Bohm, ${ }^{124}$ V. Boisvert,${ }^{75}$ T. Bold,${ }^{37}$ V. Boldea, ${ }^{25 a}$ N. M. Bolnet,${ }^{135}$ M. Bomben, ${ }^{77}$ M. Bona,${ }^{74}$ M. Boonekamp ${ }^{135}$ C. N. Booth, ${ }^{138}$ S. Bordoni,${ }^{77}$ C. Borer, ${ }^{16}$ A. Borisov, ${ }^{127}$ G. Borissov, ${ }^{70}$ I. Borjanovic, ${ }^{12 a}$ M. Borri, ${ }^{81}$ S. Borroni, ${ }^{86}$ V. Bortolotto, ${ }^{133 a, 133 b}$ K. Bos, ${ }^{104}$ D. Boscherini, ${ }^{19 a}$ M. Bosman, ${ }^{11}$ H. Boterenbrood, ${ }^{104}$ J. Bouchami, ${ }^{92}$ J. Boudreau, ${ }^{122}$ E. V. Bouhova-Thacker,${ }^{70}$ D. Boumediene, ${ }^{33}$ C. Bourdarios, ${ }^{114}$ N. Bousson, ${ }^{82}$ A. Boveia,${ }^{30}$ J. Boyd,${ }^{29}$ I. R. Boyko, ${ }^{63}$ I. Bozovic-Jelisavcic, ${ }^{12 \mathrm{~b}}$ J. Bracinik, ${ }^{17}$ P. Branchini, ${ }^{133 \mathrm{a}}$ A. Brandt, ${ }^{7}$ G. Brandt, ${ }^{117} \mathrm{O}$. Brandt, ${ }^{53}$

U. Bratzler, ${ }^{155}$ B. Brau, ${ }^{83}$ J. E. Brau, ${ }^{113}$ H. M. Braun, ${ }^{174, a}$ S. F. Brazzale, ${ }^{163 a, 163 c}$ B. Brelier,${ }^{157}$ J. Bremer, ${ }^{29}$

K. Brendlinger, ${ }^{119}$ R. Brenner, ${ }^{165}$ S. Bressler, ${ }^{171}$ D. Britton, ${ }^{52}$ F. M. Brochu, ${ }^{27}$ I. Brock, ${ }^{20}$ R. Brock,${ }^{87}$ F. Broggi, ${ }^{88 a}$ C. Bromberg, ${ }^{87}$ J. Bronner, ${ }^{98}$ G. Brooijmans,${ }^{34}$ T. Brooks,${ }^{75}$ W. K. Brooks,${ }^{31 b}$ G. Brown, ${ }^{81}$ H. Brown, ${ }^{7}$ P. A. Bruckman de Renstrom, ${ }^{38}$ D. Bruncko, ${ }^{143 \mathrm{~b}}$ R. Bruneliere,${ }^{47}$ S. Brunet, ${ }^{59}$ A. Bruni, ${ }^{19 a}$ G. Bruni, ${ }^{19 a}$ M. Bruschi, ${ }^{19 a}$ T. Buanes, ${ }^{13}$ Q. Buat,${ }^{54}$ F. Bucci, ${ }^{48}$ J. Buchanan, ${ }^{117}$ P. Buchholz,${ }^{140}$ R. M. Buckingham, ${ }^{117}$ A. G. Buckley, ${ }^{45}$ S. I. Buda, ${ }^{25 a}$ I. A. Budagov, ${ }^{63}$ B. Budick, ${ }^{107}$ V. Büscher,${ }^{80}$ L. Bugge, ${ }^{116}$ O. Bulekov, ${ }^{95}$ A. C. Bundock,${ }^{72}$ M. Bunse, ${ }^{42}$ T. Buran,${ }^{116}$ H. Burckhart, ${ }^{29}$ S. Burdin, ${ }^{72}$ T. Burgess,${ }^{13}$ S. Burke,${ }^{128}$ E. Busato, ${ }^{33}$ P. Bussey,${ }^{52}$ C. P. Buszello, ${ }^{165}$ B. Butler, ${ }^{142}$ J. M. Butler, ${ }^{21}$ C. M. Buttar, ${ }^{52}$ J. M. Butterworth, ${ }^{76}$ W. Buttinger, ${ }^{27}$ M. Byszewski, ${ }^{29}$ S. Cabrera Urbán, ${ }^{166}$ D. Caforio, ${ }^{19 a, 19 b}$ O. Cakir, ${ }^{3 a}$ P. Calafiura, ${ }^{14}$ G. Calderinin, ${ }^{77}$ P. Calfayan,${ }^{97}$ R. Calkins, ${ }^{105}$ L. P. Caloba, ${ }^{23 a}$ R. Caloi, ${ }^{131 a, 131 b}$ D. Calvet, ${ }^{33}$ S. Calvet, ${ }^{33}$ R. Camacho Toro, ${ }^{33}$ P. Camarri, ${ }^{132 a, 132 b}$ D. Cameron, ${ }^{116}$ L. M. Caminada, ${ }^{14}$ R. Caminal Armadans, ${ }^{11}$ S. Campana ${ }^{29}$ M. Campanelli, ${ }^{76}$ V. Canale, ${ }^{101 a, 101 b}$ F. Canelli, ${ }^{30, \mathrm{~h}}$ A. Canepa, ${ }^{158 \mathrm{a}}$ J. Cantero, ${ }^{79}$ R. Cantrill, ${ }^{75}$ L. Capasso, ${ }^{101 \mathrm{a}, 101 \mathrm{~b}}$ M. D. M. Capeans Garrido, ${ }^{29}$ I. Caprini, ${ }^{25 a}$ M. Caprini ${ }^{25 a}$ D. Capriotti,${ }^{98}$ M. Capua, ${ }^{36 a, 36 b}$ R. Caputo, ${ }^{80}$ R. Cardarelli, ${ }^{132 a}$ T. Carli, ${ }^{29}$ G. Carlino, ${ }^{101 a}$ L. Carminati, ${ }^{88 a, 88 b}$ B. Caron, ${ }^{84}$ S. Caron, ${ }^{103}$ E. Carquin, ${ }^{31 b}$ G. D. Carrillo Montoya, ${ }^{172}$ A. A. Carter, ${ }^{74}$ J. R. Carter, ${ }^{27}$ J. Carvalho, ${ }^{123 a, i}$ D. Casadei, ${ }^{107}$ M. P. Casado,${ }^{11}$ M. Cascella, ${ }^{121 a, 121 b}$ C. Caso, ${ }^{49 a, 49 b, a}$

A. M. Castaneda Hernandez, ${ }^{172, j}$ E. Castaneda-Miranda, ${ }^{172}$ V. Castillo Gimenez, ${ }^{166}$ N. F. Castro, ${ }^{123 a}$ G. Cataldi, ${ }^{71 a}$ 
P. Catastini, ${ }^{56}$ A. Catinaccio, ${ }^{29}$ J. R. Catmore, ${ }^{29}$ A. Cattai, ${ }^{29}$ G. Cattani,${ }^{132 a, 132 b}$ S. Caughron, ${ }^{87}$ V. Cavaliere ${ }^{164}$

P. Cavalleri, ${ }^{77}$ D. Cavalli, ${ }^{88 a}$ M. Cavalli-Sforza, ${ }^{11}$ V. Cavasinni ${ }^{121 a, 121 b}$ F. Ceradini ${ }^{133 a, 133 b}$ A. S. Cerqueira, ${ }^{23 b}$ A. Cerri, ${ }^{29}$ L. Cerrito, ${ }^{74}$ F. Cerutti, ${ }^{46}$ S. A. Cetin, ${ }^{18 b}$ A. Chafaq,${ }^{134 a}$ D. Chakraborty, ${ }^{105}$ I. Chalupkova, ${ }^{125}$ K. Chan, ${ }^{2}$ P. Chang, ${ }^{164}$ B. Chapleau, ${ }^{84}$ J. D. Chapman,${ }^{27}$ J. W. Chapman, ${ }^{86}$ E. Chareyre, ${ }^{77}$ D. G. Charlton, ${ }^{17}$ V. Chavda,${ }^{81}$ C. A. Chavez Barajas ${ }^{29}$ S. Cheatham ${ }^{84}$ S. Chekanov, ${ }^{5}$ S. V. Chekulaev, ${ }^{158 a}$ G. A. Chelkov ${ }^{63}$ M. A. Chelstowska, ${ }^{103}$ C. Chen, ${ }^{62}$ H. Chen, ${ }^{24}$ S. Chen, ${ }^{32 \mathrm{c}}$ X. Chen, ${ }^{172}$ Y. Chen, ${ }^{34}$ A. Cheplakov, ${ }^{63}$ R. Cherkaoui El Moursli, ${ }^{134 \mathrm{e}}$ V. Chernyatin, ${ }^{24}$ E. Cheu, ${ }^{6}$ S. L. Cheung, ${ }^{157}$ L. Chevalier, ${ }^{135}$ G. Chiefari, ${ }^{101 a, 101 b}$ L. Chikovani, ${ }^{50 a, a}$ J. T. Childers, ${ }^{29}$ A. Chilingarov, ${ }^{70}$ G. Chiodini, ${ }^{71 a}$ A. S. Chisholm, ${ }^{17}$ R. T. Chislett, ${ }^{76}$ A. Chitan, ${ }^{25 a}$ M. V. Chizhov, ${ }^{63}$ G. Choudalakis,${ }^{30}$ S. Chouridou, ${ }^{136}$ I. A. Christidi, ${ }^{76}$ A. Christov, ${ }^{47}$ D. Chromek-Burckhart, ${ }^{29}$ M. L. Chu, ${ }^{150}$ J. Chudoba, ${ }^{124}$ G. Ciapetti, ${ }^{131 \mathrm{a}, 131 \mathrm{~b}}$ A. K. Ciftci, ${ }^{3 \mathrm{a}}$ R. Ciftci, ${ }^{3 \mathrm{a}}$ D. Cinca, ${ }^{33}$ V. Cindro, ${ }^{73}$ C. Ciocca, ${ }^{19 a, 19 b}$ A. Ciocio, ${ }^{14}$ M. Cirilli, ${ }^{86}$ P. Cirkovic, ${ }^{12 b}$ M. Citterio, ${ }^{88 a}$ M. Ciubancan, ${ }^{25 a}$ A. Clark, ${ }^{48}$ P. J. Clark, ${ }^{45}$ R. N. Clarke, ${ }^{14}$ W. Cleland, ${ }^{122}$ J. C. Clemens, ${ }^{82}$ B. Clement, ${ }^{54}$ C. Clement, ${ }^{145 a, 145 b}$ Y. Coadou, ${ }^{82}$ M. Cobal, ${ }^{163 a, 163 \mathrm{c}}$ A. Coccaro, ${ }^{137}$ J. Cochran, ${ }^{62}$ J. G. Cogan, ${ }^{142}$ J. Coggeshall, ${ }^{164}$ E. Cogneras, ${ }^{177}$ J. Colas, ${ }^{4}$ S. Cole, ${ }^{105}$ A. P. Colijn,${ }^{104}$ N. J. Collins,${ }^{17}$ C. Collins-Tooth, ${ }^{52}$ J. Collot, ${ }^{54}$ T. Colombo, ${ }^{118 a, 118 b}$ G. Colon, ${ }^{83}$ P. Conde Muiño, ${ }^{123 a}$ E. Coniavitis, ${ }^{117}$ M. C. Conidi, ${ }^{11}$ S. M. Consonni, ${ }^{88 a, 88 b}$ V. Consorti, ${ }^{47}$ S. Constantinescu, ${ }^{25 a}$ C. Conta, ${ }^{118 a, 118 b}$ G. Conti, ${ }^{56}$ F. Conventi, ${ }^{101 \mathrm{a}, \mathrm{k}}$ M. Cooke, ${ }^{14}$ B. D. Cooper, ${ }^{76}$ A. M. Cooper-Sarkar, ${ }^{117}$ K. Copic,${ }^{14}$ T. Cornelissen, ${ }^{174}$ M. Corradi, ${ }^{19 a}$ F. Corriveau, ${ }^{84,1}$ A. Cortes-Gonzalez, ${ }^{164}$ G. Cortiana, ${ }^{98}$ G. Costa, ${ }^{88 a}$ M. J. Costa, ${ }^{166}$ D. Costanzo, ${ }^{138}$ D. Côté, ${ }^{29}$ L. Courneyea, ${ }^{168}$ G. Cowan,${ }^{75}$ C. Cowden, ${ }^{27}$ B. E. Cox ${ }^{81}$ K. Cranmer, ${ }^{107}$ F. Crescioli, ${ }^{121 a, 121 b}$ M. Cristinziani, ${ }^{20}$ G. Crosetti, ${ }^{36 a, 36 b}$ S. Crépé-Renaudin, ${ }^{54}$ C.-M. Cuciuc, ${ }^{25 a}$ C. Cuenca Almenar, ${ }^{175}$ T. Cuhadar Donszelmann, ${ }^{138}$ M. Curatolo, ${ }^{46}$ C. J. Curtis, ${ }^{17}$ C. Cuthbert, ${ }^{149}$ P. Cwetanski, ${ }^{59}$ H. Czirr, ${ }^{140}$ P. Czodrowski, ${ }^{43}$ Z. Czyczula, ${ }^{175}$ S. D'Auria, ${ }^{52}$ M. D’Onofrio, ${ }^{72}$ A. D’Orazio, ${ }^{131 a, 131 b}$ M. J. Da Cunha Sargedas De Sousa, ${ }^{123 a}$ C. Da Via, ${ }^{81}$

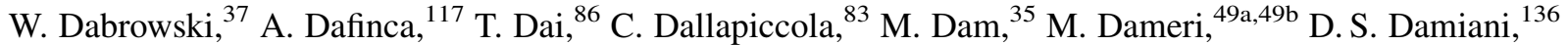
H. O. Danielsson, ${ }^{29}$ V. Dao ${ }^{48}$ G. Darbo, ${ }^{49 a}$ G. L. Darlea, ${ }^{25 b}$ J. A. Dassoulas, ${ }^{41}$ W. Davey, ${ }^{20}$ T. Davidek, ${ }^{125}$ N. Davidson, ${ }^{85}$ R. Davidson, ${ }^{70}$ E. Davies, ${ }^{117, d}$ M. Davies ${ }^{92}$ O. Davignon, ${ }^{77}$ A. R. Davison, ${ }^{76}$ Y. Davygora, ${ }^{57 a}$

E. Dawe,${ }^{141}$ I. Dawson, ${ }^{138}$ R. K. Daya-Ishmukhametova, ${ }^{22}$ K. De,${ }^{7}$ R. de Asmundis, ${ }^{101 a}$ S. De Castro, ${ }^{19 a, 19 b}$ S. De Cecco, ${ }^{77}$ J. de Graat, ${ }^{97}$ N. De Groot, ${ }^{103}$ P. de Jong, ${ }^{104}$ C. De La Taille, ${ }^{114}$ H. De la Torre,${ }^{79}$ F. De Lorenzi, ${ }^{62}$ L. de Mora, ${ }^{70}$ L. De Nooij, ${ }^{104}$ D. De Pedis, ${ }^{131 a}$ A. De Salvo, ${ }^{131 a}$ U. De Sanctis, ${ }^{163 a, 163 c}$ A. De Santo, ${ }^{148}$

J. B. De Vivie De Regie, ${ }^{114}$ G. De Zorzi, ${ }^{131 a, 131 b}$ W. J. Dearnaley ${ }^{70}$ R. Debbe,${ }^{24}$ C. Debenedetti, ${ }^{45}$ B. Dechenaux,${ }^{54}$ D. V. Dedovich ${ }^{63}$ J. Degenhardt, ${ }^{119}$ C. Del Papa,${ }^{163 a, 163 c}$ J. Del Peso, ${ }^{79}$ T. Del Prete, ${ }^{121 a, 121 b}$ T. Delemontex ${ }^{54}$ M. Deliyergiyev ${ }^{73}$ A. Dell'Acqua,${ }^{29}$ L. Dell'Asta, ${ }^{21}$ M. Della Pietra,${ }^{101 a, k}$ D. della Volpe,${ }^{101 a, 101 b}$ M. Delmastro, ${ }^{4}$ P. A. Delsart, ${ }^{54}$ C. Deluca, ${ }^{104}$ S. Demers,${ }^{175}$ M. Demichev, ${ }^{63}$ B. Demirkoz, ${ }^{11, m}$ J. Deng, ${ }^{162}$ S. P. Denisov, ${ }^{127}$ D. Derendarz, ${ }^{38}$ J. E. Derkaoui, ${ }^{134 \mathrm{~d}}$ F. Derue,${ }^{77}$ P. Dervan,${ }^{72}$ K. Desch,${ }^{20}$ E. Devetak, ${ }^{147}$ P. O. Deviveiros,${ }^{104}$ A. Dewhurst, ${ }^{128}$ B. DeWilde, ${ }^{147}$ S. Dhaliwal, ${ }^{157}$ R. Dhullipudi, ${ }^{24, n}$ A. Di Ciaccio, ${ }^{132 a, 132 b}$ L. Di Ciaccio, ${ }^{4}$ A. Di Girolamo ${ }^{29}$ B. Di Girolamo, ${ }^{29}$ S. Di Luise, ${ }^{133 a, 133 b}$ A. Di Mattia, ${ }^{172}$ B. Di Micco,${ }^{29}$ R. Di Nardo,${ }^{46}$ A. Di Simone, ${ }^{132 a, 132 b}$ R. Di Sipio, ${ }^{19 a, 19 b}$ M. A. Diaz,${ }^{31 a}$ E. B. Diehl, ${ }^{86}$ J. Dietrich, ${ }^{41}$ T. A. Dietzsch, ${ }^{57 a}$ S. Diglio, ${ }^{85}$ K. Dindar Yagci, ${ }^{39}$ J. Dingfelder, ${ }^{20}$ F. Dinut, ${ }^{25 a}$ C. Dionisi, ${ }^{131 a, 131 b}$ P. Dita, ${ }^{25 a}$ S. Dita, ${ }^{25 a}$ F. Dittus, ${ }^{29}$ F. Djama, ${ }^{82}$ T. Djobava, ${ }^{50 b}$ M. A. B. do Vale, ${ }^{23 \mathrm{c}}$ A. Do Valle Wemans, ${ }^{123 a, o}$ T. K. O. Doan, ${ }^{4}$ M. Dobbs,${ }^{84}$ R. Dobinson, ${ }^{29, a}$ D. Dobos, ${ }^{29}$ E. Dobson, ${ }^{29, p}$ J. Dodd,${ }^{34}$ C. Doglioni, ${ }^{48}$ T. Doherty, ${ }^{52}$ Y. Doi,${ }^{64, a}$ J. Dolejsi,${ }^{125}$ I. Dolenc, ${ }^{73}$ Z. Dolezal, ${ }^{125}$ B. A. Dolgoshein, ${ }^{95, a}$ T. Dohmae, ${ }^{154}$ M. Donadelli, ${ }^{23 \mathrm{~d}}$ J. Donini,${ }^{33}$ J. Dopke, ${ }^{29}$ A. Doria, ${ }^{101 a}$ A. Dos Anjos, ${ }^{172}$ A. Dotti, ${ }^{121 a, 121 b}$ M. T. Dova, ${ }^{69}$ A. D. Doxiadis, ${ }^{104}$ A. T. Doyle, ${ }^{52}$ M. Dris, ${ }^{9}$ J. Dubbert, ${ }^{98}$ S. Dube,${ }^{14}$

E. Duchovni, ${ }^{171}$ G. Duckeck, ${ }^{97}$ D. Duda, ${ }^{174}$ A. Dudarev ${ }^{29}$ F. Dudziak,${ }^{62}$ M. Dührssen, ${ }^{29}$ I. P. Duerdoth,${ }^{81}$

L. Duflot, ${ }^{114}$ M-A. Dufour ${ }^{84}$ L. Duguid, ${ }^{75}$ M. Dunford, ${ }^{29}$ H. Duran Yildiz,${ }^{3 a}$ R. Duxfield, ${ }^{138}$ M. Dwuznik, ${ }^{37}$ F. Dydak, ${ }^{29}$ M. Düren, ${ }^{51}$ J. Ebke, ${ }^{97}$ S. Eckweiler, ${ }^{80}$ K. Edmonds, ${ }^{80}$ W. Edson, ${ }^{1}$ C. A. Edwards, ${ }^{75}$ N. C. Edwards, ${ }^{52}$ W. Ehrenfeld, ${ }^{41}$ T. Eifert, ${ }^{142}$ G. Eigen, ${ }^{13}$ K. Einsweiler, ${ }^{14}$ E. Eisenhandler, ${ }^{74}$ T. Ekelof,,${ }^{165}$ M. El Kacimi, ${ }^{134 c}$ M. Ellert, ${ }^{165}$ S. Elles ${ }^{4}$ F. Ellinghaus, ${ }^{80}$ K. Ellis, ${ }^{74}$ N. Ellis, ${ }^{29}$ J. Elmsheuser, ${ }^{97}$ M. Elsing, ${ }^{29}$ D. Emeliyanov, ${ }^{128}$

R. Engelmann, ${ }^{147}$ A. Engl, ${ }^{97}$ B. Epp, ${ }^{60}$ J. Erdmann, ${ }^{53}$ A. Ereditato, ${ }^{16}$ D. Eriksson, ${ }^{145 a}$ J. Ernst, ${ }^{1}$ M. Ernst, ${ }^{24}$ J. Ernwein, ${ }^{135}$ D. Errede, ${ }^{164}$ S. Errede, ${ }^{164}$ E. Ertel, ${ }^{80}$ M. Escalier, ${ }^{114}$ H. Esch, ${ }^{42}$ C. Escobar, ${ }^{122}$ X. Espinal Curull, ${ }^{11}$ B. Esposito, ${ }^{46}$ F. Etienne, ${ }^{82}$ A. I. Etienvre, ${ }^{135}$ E. Etzion, ${ }^{152}$ D. Evangelakou, ${ }^{53}$ H. Evans,${ }^{59}$ L. Fabbri, ${ }^{19 a, 19 b}$ C. Fabre,${ }^{29}$

R. M. Fakhrutdinov, ${ }^{127}$ S. Falciano, ${ }^{131 a}$ Y. Fang, ${ }^{172}$ M. Fanti, ${ }^{88 a}, 88 b$ A. Farbin, ${ }^{7}$ A. Farilla, ${ }^{133 a}$ J. Farley, ${ }^{147}$

T. Farooque, ${ }^{157}$ S. Farrell, ${ }^{162}$ S. M. Farrington, ${ }^{169}$ P. Farthouat, ${ }^{29}$ F. Fassi, ${ }^{166}$ P. Fassnacht, ${ }^{29}$ D. Fassouliotis, ${ }^{8}$ B. Fatholahzadeh, ${ }^{157}$ A. Favareto, ${ }^{88 a, 88 b}$ L. Fayard, ${ }^{114}$ S. Fazio, ${ }^{36 a, 36 b}$ R. Febbraro, ${ }^{33}$ P. Federic, ${ }^{143 a}$ O. L. Fedin, ${ }^{120}$ 
W. Fedorko, ${ }^{87}$ M. Fehling-Kaschek, ${ }^{47}$ L. Feligioni, ${ }^{82}$ D. Fellmann, ${ }^{5}$ C. Feng, ${ }^{32 d}$ E. J. Feng, ${ }^{5}$ A. B. Fenyuk, ${ }^{127}$ J. Ferencei, ${ }^{143 \mathrm{~b}}$ W. Fernando, ${ }^{5}$ S. Ferrag, ${ }^{52}$ J. Ferrando, ${ }^{52}$ V. Ferrara, ${ }^{41}$ A. Ferrari, ${ }^{165}$ P. Ferrari, ${ }^{104}$ R. Ferrari, ${ }^{118 a}$ D. E. Ferreira de Lima ${ }^{52}$ A. Ferrer, ${ }^{166}$ D. Ferrere,${ }^{48}$ C. Ferretti ${ }^{86}$ A. Ferretto Parodi,,${ }^{49,49 b}$ M. Fiascaris, ${ }^{30}$ F. Fiedler ${ }^{80}$ A. Filipčič ${ }^{73}$ F. Filthaut, ${ }^{103}$ M. Fincke-Keeler, ${ }^{168}$ M. C. N. Fiolhais,${ }^{123 a, i}$ L. Fiorini, ${ }^{166}$ A. Firan, ${ }^{39}$ G. Fischer, ${ }^{41}$ M. J. Fisher, ${ }^{108}$ M. Flechl,${ }^{47}$ I. Fleck, ${ }^{140}$ J. Fleckner, ${ }^{80}$ P. Fleischmann, ${ }^{173}$ S. Fleischmann, ${ }^{174}$ T. Flick, ${ }^{174}$ A. Floderus, ${ }^{78}$ L. R. Flores Castillo, ${ }^{172}$ M. J. Flowerdew, ${ }^{98}$ T. Fonseca Martin, ${ }^{16}$ A. Formica, ${ }^{135}$ A. Forti, ${ }^{81}$ D. Fortin, ${ }^{158 \mathrm{a}}$ D. Fournier, ${ }^{114}$ H. Fox ${ }^{70}$ P. Francavilla, ${ }^{11}$ M. Franchini, ${ }^{19 a, 19 b}$ S. Franchino, ${ }^{118 a, 118 b}$ D. Francis,${ }^{29}$ T. Frank,${ }^{171}$ S. Franz,${ }^{29}$ M. Fraternali, ${ }^{118 a, 118 b}$ S. Fratina, ${ }^{119}$ S. T. French,${ }^{27}$ C. Friedrich,${ }^{41}$ F. Friedrich,${ }^{43}$ R. Froeschl, ${ }^{29}$ D. Froidevaux, ${ }^{29}$ J. A. Frost,${ }^{27}$ C. Fukunaga, ${ }^{155}$ E. Fullana Torregrosa, ${ }^{29}$ B. G. Fulsom, ${ }^{142}$ J. Fuster, ${ }^{166}$ C. Gabaldon, ${ }^{29}$ O. Gabizon, ${ }^{171}$ T. Gadfort, ${ }^{24}$ S. Gadomski, ${ }^{48}$ G. Gagliardi, ${ }^{49 a, 49 b}$ P. Gagnon, ${ }^{59}$ C. Galea,${ }^{97}$ E. J. Gallas, ${ }^{117}$ V. Gallo, ${ }^{16}$ B. J. Gallop, ${ }^{128}$ P. Gallus, ${ }^{124}$ K. K. Gan, ${ }^{108}$ Y. S. Gao, ${ }^{142, f}$ A. Gaponenko, ${ }^{14}$ F. Garberson, ${ }^{175}$ M. Garcia-Sciveres, ${ }^{14}$ C. García, ${ }^{166}$ J. E. García Navarro, ${ }^{166}$ R. W. Gardner, ${ }^{30}$ N. Garelli, ${ }^{29}$ H. Garitaonandia, ${ }^{104}$ V. Garonne, ${ }^{29}$ C. Gatti, ${ }^{46}$ G. Gaudio, ${ }^{118 a}$ B. Gaur, ${ }^{140}$ L. Gauthier, ${ }^{135}$ P. Gauzzi, ${ }^{131 a, 131 b}$ I. L. Gavrilenko, ${ }^{93}$ C. Gay, ${ }^{167}$ G. Gaycken, ${ }^{20}$ E. N. Gazis, ${ }^{9}$ P. Ge, ${ }^{32 \mathrm{~d}}$ Z. Gecse, ${ }^{167}$ C. N. P. Gee, ${ }^{128}$ D. A. A. Geerts, ${ }^{104}$ Ch. Geich-Gimbel, ${ }^{20}$ K. Gellerstedt, ${ }^{145 a, 145 b}$ C. Gemme, ${ }^{49 a}$ A. Gemmell, ${ }^{52}$ M. H. Genest, ${ }^{54}$ S. Gentile, ${ }^{131 a, 131 b}$ M. George, ${ }^{53}$ S. George, ${ }^{75}$ P. Gerlach, ${ }^{174}$ A. Gershon, ${ }^{152}$ C. Geweniger, ${ }^{57 a}$ H. Ghazlane, ${ }^{134 b}$ N. Ghodbane, ${ }^{33}$ B. Giacobbe, ${ }^{19 \mathrm{a}}$ S. Giagu, ${ }^{131 \mathrm{a}, 131 \mathrm{~b}}$ V. Giakoumopoulou, ${ }^{8}$ V. Giangiobbe, ${ }^{11}$ F. Gianotti, ${ }^{29}$ B. Gibbard ${ }^{24}$ A. Gibson, ${ }^{157}$ S. M. Gibson, ${ }^{29}$ D. Gillberg, ${ }^{28}$ A. R. Gillman, ${ }^{128}$ D. M. Gingrich,${ }^{2, \mathrm{e}}$ J. Ginzburg, ${ }^{152}$ N. Giokaris,${ }^{8}$ M. P. Giordani, ${ }^{163 \mathrm{c}}$ R. Giordano, ${ }^{101 \mathrm{a}, 101 \mathrm{~b}}$ F. M. Giorgi, ${ }^{15}$ P. Giovannini, ${ }^{98}$ P. F. Giraud,${ }^{135}$ D. Giugni, ${ }^{88 \mathrm{a}}$ M. Giunta, ${ }^{92}$ P. Giusti, ${ }^{19 a}$ B. K. Gjelsten, ${ }^{16}$ L. K. Gladilin, ${ }^{96}$ C. Glasman, ${ }^{79}$ J. Glatzer, ${ }^{47}$ A. Glazov, ${ }^{41}$ K. W. Glitza, ${ }^{174}$ G. L. Glonti, ${ }^{63}$ J. R. Goddard, ${ }^{74}$ J. Godfrey, ${ }^{141}$ J. Godlewski, ${ }^{29}$ M. Goebel, ${ }^{41}$ T. Göpfert, ${ }^{43}$ C. Goeringer ${ }^{80}$ C. Gössling, ${ }^{42}$ S. Goldfarb, ${ }^{86}$ T. Golling, ${ }^{175}$ A. Gomes, ${ }^{123 a, c}$ L. S. Gomez Fajardo, ${ }^{41}$ R. Gonçalo, ${ }^{75}$

J. Goncalves Pinto Firmino Da Costa, ${ }^{41}$ L. Gonella, ${ }^{20}$ S. Gonzalez, ${ }^{172}$ S. González de la Hoz,${ }^{166}$ G. Gonzalez Parra, ${ }^{11}$ M. L. Gonzalez Silva, ${ }^{26}$ S. Gonzalez-Sevilla, ${ }^{48}$ J. J. Goodson, ${ }^{147}$ L. Goossens,${ }^{29}$ P. A. Gorbounov, ${ }^{94}$ H. A. Gordon, ${ }^{24}$ I. Gorelov, ${ }^{102}$ G. Gorfine, ${ }^{174}$ B. Gorini, ${ }^{29}$ E. Gorini, ${ }^{71 a, 71 b}$ A. Gorišek,${ }^{73}$ E. Gornicki, ${ }^{38}$ B. Gosdzik, ${ }^{41}$ A. T. Goshaw, ${ }^{5}$ M. Gosselink, ${ }^{104}$ M. I. Gostkin, ${ }^{63}$ I. Gough Eschrich, ${ }^{162}$ M. Gouighri, ${ }^{134 a}$ D. Goujdami, ${ }^{134 c}$ M. P. Goulette, ${ }^{48}$ A. G. Goussiou, ${ }^{137}$ C. Goy, ${ }^{4}$ S. Gozpinar, ${ }^{22}$ I. Grabowska-Bold,${ }^{37}$ P. Grafström, ${ }^{19 a}{ }^{19 b}$ K-J. Grahn, ${ }^{41}$ F. Grancagnolo, ${ }^{71 a}$ S. Grancagnolo, ${ }^{15}$ V. Grassi, ${ }^{147}$ V. Gratchev, ${ }^{120}$ N. Grau,${ }^{34}$ H. M. Gray, ${ }^{29}$ J. A. Gray, ${ }^{147}$ E. Graziani, ${ }^{133 a}$ O. G. Grebenyuk, ${ }^{120}$ T. Greenshaw, ${ }^{72}$ Z. D. Greenwood,${ }^{24, \mathrm{n}}$ K. Gregersen,${ }^{35}$ I. M. Gregor, ${ }^{41}$ P. Grenier, ${ }^{142}$ J. Griffiths ${ }^{7}$ N. Grigalashvili, ${ }^{63}$ A. A. Grillo, ${ }^{136}$ S. Grinstein, ${ }^{11}$ Ph. Gris, ${ }^{33}$ Y. V. Grishkevich,${ }^{96}$ J.-F. Grivaz, ${ }^{114}$ E. Gross, ${ }^{171}$ J. Grosse-Knetter, ${ }^{53}$ J. Groth-Jensen, ${ }^{171}$ K. Grybel, ${ }^{140}$ D. Guest, ${ }^{175}$ C. Guicheney, ${ }^{33}$

S. Guindon, ${ }^{53}$ U. Gul,${ }^{52}$ H. Guler, ${ }^{84, q}$ J. Gunther, ${ }^{124}$ B. Guo,${ }^{157}$ J. Guo,${ }^{34}$ P. Gutierrez, ${ }^{110}$ N. Guttman, ${ }^{152}$ O. Gutzwiller, ${ }^{172}$ C. Guyot, ${ }^{135}$ C. Gwenlan, ${ }^{17}$ C. B. Gwilliam, ${ }^{72}$ A. Haas, ${ }^{142}$ S. Haas, ${ }^{29}$ C. Haber, ${ }^{14}$ H. K. Hadavand, ${ }^{39}$ D. R. Hadley, ${ }^{17}$ P. Haefner, ${ }^{20}$ F. Hahn, ${ }^{29}$ S. Haider ${ }^{29}$ Z. Hajduk, ${ }^{38}$ H. Hakobyan, ${ }^{176}$ D. Hall, ${ }^{117}$ J. Haller, ${ }^{53}$ K. Hamacher, ${ }^{174}$ P. Hamal, ${ }^{112}$ M. Hamer, ${ }^{53}$ A. Hamilton, ${ }^{144 b, r}$ S. Hamilton, ${ }^{160}$ L. Han,${ }^{32 b}$ K. Hanagaki, ${ }^{115}$ K. Hanawa, ${ }^{159}$ M. Hance, ${ }^{14}$ C. Handel, ${ }^{80}$ P. Hanke, ${ }^{57 a}$ J. R. Hansen, ${ }^{35}$ J. B. Hansen, ${ }^{35}$ J. D. Hansen, ${ }^{35}$ P. H. Hansen, ${ }^{35}$ P. Hansson, ${ }^{142}$ K. Hara, ${ }^{159}$ G. A. Hare, ${ }^{136}$ T. Harenberg, ${ }^{174}$ S. Harkusha, ${ }^{89}$ D. Harper, ${ }^{86}$ R. D. Harrington, ${ }^{45}$ O. M. Harris, ${ }^{137}$ J. Hartert,${ }^{47}$ F. Hartjes,${ }^{104}$ T. Haruyama, ${ }^{64}$ A. Harvey ${ }^{55}$ S. Hasegawa, ${ }^{100}$ Y. Hasegawa,${ }^{139}$ S. Hassani, ${ }^{135}$ S. Haug,${ }^{16}$ M. Hauschild,${ }^{29}$ R. Hauser,${ }^{87}$ M. Havranek,${ }^{20}$ C. M. Hawkes, ${ }^{17}$ R. J. Hawkings,${ }^{29}$ A. D. Hawkins, ${ }^{78}$ D. Hawkins, ${ }^{162}$ T. Hayakawa, ${ }^{65}$ T. Hayashi, ${ }^{159}$ D. Hayden, ${ }^{75}$ C. P. Hays,${ }^{117}$ H. S. Hayward ${ }^{72}$ S. J. Haywood, ${ }^{128}$ M. He ${ }^{32 d}$ S. J. Head,${ }^{17}$ V. Hedberg,${ }^{78}$ L. Heelan, ${ }^{7}$ S. Heim,${ }^{87}$ B. Heinemann, ${ }^{14}$ S. Heisterkamp,${ }^{35}$ L. Helary, ${ }^{21}$ C. Heller,${ }^{97}$ M. Heller, ${ }^{29}$ S. Hellman, ${ }^{145 a, 145 b}$ D. Hellmich, ${ }^{20}$ C. Helsens, ${ }^{11}$ R. C. W. Henderson, ${ }^{70}$ M. Henke, ${ }^{57 a}$ A. Henrichs, ${ }^{53}$ A. M. Henriques Correia, ${ }^{29}$ S. Henrot-Versille, ${ }^{114}$ C. Hensel,${ }^{53}$ T. Henß,${ }^{174}$

C. M. Hernandez, ${ }^{7}$ Y. Hernández Jiménez, ${ }^{166}$ R. Herrberg,,${ }^{15}$ G. Herten,${ }^{47}$ R. Hertenberger, ${ }^{97}$ L. Hervas, ${ }^{29}$ G. G. Hesketh, ${ }^{76}$ N. P. Hessey, ${ }^{104}$ E. Higón-Rodriguez, ${ }^{166}$ J. C. Hill, ${ }^{27}$ K. H. Hiller, ${ }^{41}$ S. Hillert, ${ }^{20}$ S. J. Hillier, ${ }^{17}$

I. Hinchliffe, ${ }^{14}$ E. Hines, ${ }^{119}$ M. Hirose,${ }^{115}$ F. Hirsch,${ }^{42}$ D. Hirschbuehl, ${ }^{174}$ J. Hobbs,${ }^{147}$ N. Hod, ${ }^{152}$

M. C. Hodgkinson, ${ }^{138}$ P. Hodgson, ${ }^{138}$ A. Hoecker, ${ }^{29}$ M. R. Hoeferkamp, ${ }^{102}$ J. Hoffman, ${ }^{39}$ D. Hoffmann, ${ }^{82}$ M. Hohlfeld ${ }^{80}$ M. Holder, ${ }^{140}$ S. O. Holmgren, ${ }^{145 a}$ T. Holy, ${ }^{126}$ J. L. Holzbauer,${ }^{87}$ T. M. Hong, ${ }^{119}$

L. Hooft van Huysduynen, ${ }^{107}$ S. Horner, ${ }^{47}$ J-Y. Hostachy,${ }^{54}$ S. Hou, ${ }^{150}$ A. Hoummada, ${ }^{134 a}$ J. Howard, ${ }^{117}$ J. Howarth, ${ }^{81}$ I. Hristova, ${ }^{15}$ J. Hrivnac, ${ }^{114}$ T. Hryn'ova, ${ }^{4}$ P. J. Hsu, ${ }^{80}$ S.-C. Hsu, ${ }^{14}$ D. Hu, ${ }^{34}$ Z. Hubacek, ${ }^{126}$ F. Hubaut, ${ }^{82}$ F. Huegging, ${ }^{20}$ A. Huettmann, ${ }^{41}$ T. B. Huffman, ${ }^{117}$ E. W. Hughes,${ }^{34}$ G. Hughes,${ }^{70}$ M. Huhtinen, ${ }^{29}$ M. Hurwitz, ${ }^{14}$ 
U. Husemann, ${ }^{41}$ N. Huseynov, ${ }^{63, s}$ J. Huston, ${ }^{87}$ J. Huth,${ }^{56}$ G. Iacobucci, ${ }^{48}$ G. Iakovidis, ${ }^{9}$ M. Ibbotson, ${ }^{81}$ I. Ibragimov, ${ }^{140} \mathrm{~L}$. Iconomidou-Fayard, ${ }^{114} \mathrm{~J}$. Idarraga, ${ }^{114} \mathrm{P}$. Iengo, ${ }^{101 \mathrm{a}} \mathrm{O}$. Igonkina, ${ }^{104} \mathrm{Y}$. Ikegami, ${ }^{64} \mathrm{M}$. Ikeno, ${ }^{64}$ D. Iliadis, ${ }^{153} \mathrm{~N}$. Ilic, ${ }^{157} \mathrm{~T}$. Ince, ${ }^{20} \mathrm{~J}$. Inigo-Golfin, ${ }^{29} \mathrm{P}$. Ioannou, ${ }^{8} \mathrm{M}$. Iodice, ${ }^{133 \mathrm{a}} \mathrm{K}$. Iordanidou, ${ }^{8} \mathrm{~V}$. Ippolito, ${ }^{131 \mathrm{a}, 131 \mathrm{~b}}$

A. Irles Quiles, ${ }^{166} \mathrm{C}$. Isaksson, ${ }^{165} \mathrm{M}$. Ishino, ${ }^{66} \mathrm{M}$. Ishitsuka, ${ }^{156} \mathrm{R}$. Ishmukhametov, ${ }^{39} \mathrm{C}$. Issever, ${ }^{117} \mathrm{~S}$. Istin, ${ }^{18 \mathrm{a}}$ A. V. Ivashin, ${ }^{127}$ W. Iwanski, ${ }^{38}$ H. Iwasaki, ${ }^{64}$ J. M. Izen, ${ }^{40}$ V. Izzo, ${ }^{101 a}$ B. Jackson, ${ }^{119}$ J. N. Jackson, ${ }^{72}$ P. Jackson, ${ }^{142}$ M. R. Jaekel, ${ }^{29}$ V. Jain, ${ }^{59}$ K. Jakobs ${ }^{47}$ S. Jakobsen, ${ }^{35}$ T. Jakoubek, ${ }^{124}$ J. Jakubek, ${ }^{126}$ D. K. Jana, ${ }^{110}$ E. Jansen, ${ }^{76}$ H. Jansen, ${ }^{29}$ A. Jantsch, ${ }^{98}$ M. Janus, ${ }^{47}$ G. Jarlskog, ${ }^{78}$ L. Jeanty, ${ }^{56}$ I. Jen-La Plante,${ }^{30}$ D. Jennens, ${ }^{85}$ P. Jenni, ${ }^{29}$

A. E. Loevschall-Jensen, ${ }^{35}$ P. Jež, ${ }^{35}$ S. Jézéquel, ${ }^{4}$ M. K. Jha, ${ }^{19 a}$ H. Ji, ${ }^{172}$ W. Ji ${ }^{80}$ J. Jia, ${ }^{147}$ Y. Jiang, ${ }^{32 b}$

M. Jimenez Belenguer, ${ }^{41}$ S. Jin, ${ }^{32 a}$ O. Jinnouchi, ${ }^{156}$ M. D. Joergensen, ${ }^{35}$ D. Joffe, ${ }^{39}$ M. Johansen, ${ }^{145 a, 145 b}$ K. E. Johansson, ${ }^{145 a}$ P. Johansson, ${ }^{138}$ S. Johnert, ${ }^{41}$ K. A. Johns, ${ }^{6}$ K. Jon-And,${ }^{145 a, 145 b}$ G. Jones,${ }^{169}$ R. W. L. Jones, ${ }^{70}$

T. J. Jones, ${ }^{72}$ C. Joram,${ }^{29}$ P. M. Jorge, ${ }^{123 a}$ K. D. Joshi, ${ }^{81}$ J. Jovicevic, ${ }^{146}$ T. Jovin, ${ }^{12 b}$ X. Ju, ${ }^{172}$ C. A. Jung,${ }^{42}$ R. M. Jungst,${ }^{29}$ V. Juranek, ${ }^{124}$ P. Jussel,${ }^{60}$ A. Juste Rozas, ${ }^{11}$ S. Kabana,${ }^{16}$ M. Kaci, ${ }^{166}$ A. Kaczmarska ${ }^{38}$ P. Kadlecik,${ }^{35}$ M. Kado,${ }^{114}$ H. Kagan, ${ }^{108}$ M. Kagan ${ }^{56}$ E. Kajomovitz, ${ }^{151}$ S. Kalinin,${ }^{174}$ L. V. Kalinovskaya, ${ }^{63}$ S. Kama, ${ }^{39}$ N. Kanaya ${ }^{154}$ M. Kaneda, ${ }^{29}$ S. Kaneti ${ }^{27}$ T. Kanno, ${ }^{156}$ V. A. Kantserov, ${ }^{95}$ J. Kanzaki, ${ }^{64}$ B. Kaplan, ${ }^{107}$ A. Kapliy, ${ }^{30}$ J. Kaplon, ${ }^{29}$ D. Kar, ${ }^{52}$ M. Karagounis, ${ }^{20}$ K. Karakostas, ${ }^{9}$ M. Karnevskiy, ${ }^{41}$ V. Kartvelishvili, ${ }^{70}$ A. N. Karyukhin, ${ }^{127}$ L. Kashif, ${ }^{172}$ G. Kasieczka, ${ }^{57 b}$ R. D. Kass, ${ }^{108}$ A. Kastanas, ${ }^{13}$ M. Kataoka, ${ }^{4}$ Y. Kataoka, ${ }^{154}$ E. Katsoufis, ${ }^{9}$ J. Katzy, ${ }^{41}$ V. Kaushik, ${ }^{6}$ K. Kawagoe, ${ }^{68}$ T. Kawamoto, ${ }^{154}$ G. Kawamura ${ }^{80}$ M. S. Kayl, ${ }^{104}$ S. Kazama, ${ }^{154}$ V. A. Kazanin, ${ }^{106}$ M. Y. Kazarinov, ${ }^{63}$ R. Keeler, ${ }^{168}$ R. Kehoe, ${ }^{39}$ M. Keil,${ }^{53}$ G. D. Kekelidze, ${ }^{63}$ J. S. Keller, ${ }^{137}$ M. Kenyon, ${ }^{52}$ O. Kepka, ${ }^{124}$ N. Kerschen ${ }^{29}$ B. P. Kerševan, ${ }^{73}$ S. Kersten, ${ }^{174}$ K. Kessoku, ${ }^{154}$ J. Keung, ${ }^{157}$ F. Khalil-zada, ${ }^{10}$ H. Khandanyan, ${ }^{145 a, 145 b}$ A. Khanov, ${ }^{111}$ D. Kharchenko, ${ }^{63}$ A. Khodinov, ${ }^{95}$ A. Khomich, ${ }^{57 a}$ T. J. Khoo, ${ }^{27}$ G. Khoriauli, ${ }^{20}$ A. Khoroshilov, ${ }^{174}$ V. Khovanskiy, ${ }^{94}$ E. Khramov, ${ }^{63}$ J. Khubua ${ }^{50 b}$ H. Kim, ${ }^{145 a, 145 b}$ S. H. Kim, ${ }^{159}$ N. Kimura, ${ }^{170}$ O. Kind, ${ }^{15}$ B. T. King ${ }^{72}$

M. King, ${ }^{65}$ R. S. B. King, ${ }^{117}$ J. Kirk, ${ }^{128}$ A.E. Kiryunin, ${ }^{98}$ T. Kishimoto, ${ }^{65}$ D. Kisielewska ${ }^{37}$ T. Kitamura ${ }^{65}$ T. Kittelmann, ${ }^{122}$ K. Kiuchi, ${ }^{159}$ E. Kladiva, ${ }^{143 b}$ M. Klein, ${ }^{72}$ U. Klein, ${ }^{72}$ K. Kleinknecht, ${ }^{80}$ M. Klemetti, ${ }^{84}$ A. Klier, ${ }^{171}$

P. Klimek, ${ }^{145 a, 145 b}$ A. Klimentov, ${ }^{24}$ R. Klingenberg, ${ }^{42}$ J. A. Klinger, ${ }^{81}$ E. B. Klinkby, ${ }^{35}$ T. Klioutchnikova, ${ }^{29}$

P. F. Klok, ${ }^{103}$ S. Klous, ${ }^{104}$ E.-E. Kluge, ${ }^{57 a}$ T. Kluge, ${ }^{72}$ P. Kluit,,${ }^{104}$ S. Kluth, ${ }^{98}$ N. S. Knecht, ${ }^{157}$ E. Kneringer, ${ }^{60}$ E.B.F.G. Knoops, ${ }^{82}$ A. Knue,${ }^{53}$ B. R. Ko, ${ }^{44}$ T. Kobayashi, ${ }^{154}$ M. Kobel,${ }^{43}$ M. Kocian, ${ }^{142}$ P. Kodys, ${ }^{125}$ K. Köneke, ${ }^{29}$ A. C. König, ${ }^{103}$ S. Koenig, ${ }^{80}$ L. Köpke,${ }^{80}$ F. Koetsveld, ${ }^{103}$ P. Koevesarki, ${ }^{20}$ T. Koffas,${ }^{28}$ E. Koffeman, ${ }^{104}$

L. A. Kogan, ${ }^{117}$ S. Kohlmann, ${ }^{174}$ F. Kohn, ${ }^{53}$ Z. Kohout, ${ }^{126}$ T. Kohriki, ${ }^{64}$ T. Koi, ${ }^{142}$ G. M. Kolachev, ${ }^{106, a}$

H. Kolanoski, ${ }^{15}$ V. Kolesnikov, ${ }^{63}$ I. Koletsou, ${ }^{88 a}$ J. Koll, ${ }^{87}$ M. Kollefrath, ${ }^{47}$ A. A. Komar, ${ }^{93}$ Y. Komori, ${ }^{154}$ T. Kondo ${ }^{64}$

T. Kono, ${ }^{41, t}$ A. I. Kononov, ${ }^{47}$ R. Konoplich, ${ }^{107, u}$ N. Konstantinidis, ${ }^{76}$ S. Koperny, ${ }^{37}$ K. Korcyl,${ }^{38}$ K. Kordas, ${ }^{153}$

A. Korn, ${ }^{117}$ A. Korol, ${ }^{106}$ I. Korolkov, ${ }^{11}$ E. V. Korolkova, ${ }^{138}$ V. A. Korotkov, ${ }^{127}$ O. Kortner, ${ }^{98}$ S. Kortner, ${ }^{98}$ V. V. Kostyukhin, ${ }^{20}$ S. Kotov, ${ }^{98}$ V. M. Kotov ${ }^{63}$ A. Kotwal, ${ }^{44}$ C. Kourkoumelis, ${ }^{8}$ V. Kouskoura, ${ }^{153}$ A. Koutsman, ${ }^{158 a}$

R. Kowalewski, ${ }^{168}$ T.Z. Kowalski, ${ }^{37}$ W. Kozanecki, ${ }^{135}$ A. S. Kozhin, ${ }^{127}$ V. Kral, ${ }^{126}$ V. A. Kramarenko, ${ }^{96}$ G. Kramberger, ${ }^{73}$ M. W. Krasny, ${ }^{77}$ A. Krasznahorkay, ${ }^{107}$ J. K. Kraus ${ }^{20}$ S. Kreiss, ${ }^{107}$ F. Krejci, ${ }^{126}$ J. Kretzschmar, ${ }^{72}$ N. Krieger, ${ }^{53}$ P. Krieger, ${ }^{157}$ K. Kroeninger ${ }^{53}$ H. Kroha, ${ }^{98}$ J. Kroll, ${ }^{119}$ J. Kroseberg, ${ }^{20}$ J. Krstic, ${ }^{12 a}$ U. Kruchonak, ${ }^{63}$ H. Krüger, ${ }^{20}$ T. Kruker, ${ }^{16}$ N. Krumnack, ${ }^{62}$ Z. V. Krumshteyn, ${ }^{63}$ T. Kubota, ${ }^{85}$ S. Kuday, ${ }^{3 a}$ S. Kuehn, ${ }^{47}$ A. Kugel,${ }^{57 \mathrm{c}}$

T. Kuhl, ${ }^{41}$ D. Kuhn, ${ }^{60}$ V. Kukhtin, ${ }^{63}$ Y. Kulchitsky, ${ }^{89}$ S. Kuleshov, ${ }^{31 b}$ C. Kummer, ${ }^{97}$ M. Kuna, ${ }^{77}$ J. Kunkle, ${ }^{119}$

A. Kupco, ${ }^{124}$ H. Kurashige, ${ }^{65}$ M. Kurata, ${ }^{159}$ Y. A. Kurochkin, ${ }^{89}$ V. Kus, ${ }^{124}$ E. S. Kuwertz, ${ }^{146}$ M. Kuze, ${ }^{156}$ J. Kvita, ${ }^{141}$

R. Kwee, ${ }^{15}$ A. La Rosa,${ }^{48}$ L. La Rotonda, ${ }^{36 a, 36 b}$ L. Labarga, ${ }^{79}$ J. Labbe, ${ }^{4}$ S. Lablak, ${ }^{134 a}$ C. Lacasta, ${ }^{166}$

F. Lacava, ${ }^{131 \mathrm{a}, 131 \mathrm{~b}}$ H. Lacker, ${ }^{15}$ D. Lacour, ${ }^{77}$ V. R. Lacuesta, ${ }^{166}$ E. Ladygin, ${ }^{63}$ R. Lafaye,${ }^{4}$ B. Laforge,${ }^{77}$ T. Lagouri, ${ }^{79}$ S. Lai,${ }^{47}$ E. Laisne,${ }^{54}$ M. Lamanna, ${ }^{29}$ L. Lambourne, ${ }^{76}$ C. L. Lampen, ${ }^{6}$ W. Lampl,${ }^{6}$ E. Lancon, ${ }^{135}$ U. Landgraf,${ }^{47}$ M. P. J. Landon, ${ }^{74}$ J. L. Lane, ${ }^{81}$ V. S. Lang, ${ }^{57 a}$ C. Lange, ${ }^{41}$ A. J. Lankford, ${ }^{162}$ F. Lanni, ${ }^{24}$ K. Lantzsch, ${ }^{174}$ S. Laplace, ${ }^{77}$ C. Lapoire, ${ }^{20}$ J. F. Laporte, ${ }^{135}$ T. Lari ${ }^{88 \mathrm{a}}$ A. Larner, ${ }^{117}$ M. Lassnig, ${ }^{29}$ P. Laurelli, ${ }^{46}$ V. Lavorini,,${ }^{36 a, 36 \mathrm{~b}} \mathrm{~W}$. Lavrijsen, ${ }^{14}$ P. Laycock, ${ }^{72}$ O. Le Dortz, ${ }^{77}$ E. Le Guirriec, ${ }^{82}$ C. Le Maner, ${ }^{157}$ E. Le Menedeu, ${ }^{11}$ T. LeCompte, ${ }^{5}$

F. Ledroit-Guillon, ${ }^{54}$ H. Lee, ${ }^{104}$ J. S. H. Lee, ${ }^{115}$ S. C. Lee, ${ }^{150}$ L. Lee, ${ }^{175}$ M. Lefebvre, ${ }^{168}$ M. Legendre, ${ }^{135}$ F. Legger,${ }^{97}$ C. Leggett, ${ }^{14}$ M. Lehmacher, ${ }^{20}$ G. Lehmann Miotto, ${ }^{29}$ X. Lei, ${ }^{6}$ M. A. L. Leite, ${ }^{23 d}$ R. Leitner, ${ }^{125}$ D. Lellouch,${ }^{171}$ B. Lemmer, ${ }^{53}$ V. Lendermann, ${ }^{57 \mathrm{a}}$ K. J. C. Leney, ${ }^{144 \mathrm{~b}}$ T. Lenz, ${ }^{104}$ G. Lenzen, ${ }^{174}$ B. Lenzi, ${ }^{29}$ K. Leonhardt, ${ }^{43}$ S. Leontsinis, ${ }^{9}$ F. Lepold,${ }^{57 a}$ C. Leroy,${ }^{92}$ J-R. Lessard, ${ }^{168}$ C. G. Lester, ${ }^{27}$ C. M. Lester, ${ }^{119}$ J. Levêque, ${ }^{4}$ D. Levin, ${ }^{86}$ L. J. Levinson, ${ }^{171}$ A. Lewis, ${ }^{17}$ G. H. Lewis, ${ }^{107}$ A. M. Leyko, ${ }^{20}$ M. Leyton, ${ }^{15}$ B. Li ${ }^{82} \mathrm{H}$. Li, ${ }^{172, v} \mathrm{~S}$. Li,${ }^{32 b, w}$ X. Li, ${ }^{86}$ Z. Liang, ${ }^{117, \mathrm{x}}$ H. Liao, ${ }^{33}$ B. Liberti, ${ }^{132 \mathrm{a}}$ P. Lichard ${ }^{29}$ M. Lichtnecker, ${ }^{97} \mathrm{~K}$. Lie,${ }^{164}$ W. Liebig, ${ }^{13}$ C. Limbach, ${ }^{20}$

A. Limosani, ${ }^{85}$ M. Limper, ${ }^{61}$ S. C. Lin,${ }^{150, y}$ F. Linde, ${ }^{104}$ J. T. Linnemann, ${ }^{87}$ E. Lipeles, ${ }^{119}$ A. Lipniacka, ${ }^{13}$ 
T. M. Liss, ${ }^{164}$ D. Lissauer, ${ }^{24}$ A. Lister, ${ }^{48}$ A. M. Litke, ${ }^{136}$ C. Liu, ${ }^{28}$ D. Liu, ${ }^{150}$ H. Liu, ${ }^{86}$ J. B. Liu, ${ }^{86}$ L. Liu, ${ }^{86}$ M. Liu,${ }^{32 b}$ Y. Liu, ${ }^{32 b}$ M. Livan, ${ }^{118 a, 118 b}$ S. S. A. Livermore, ${ }^{117}$ A. Lleres, ${ }^{54}$ J. Llorente Merino, ${ }^{79}$ S. L. Lloyd, ${ }^{74}$

E. Lobodzinska, ${ }^{41}$ P. Loch, ${ }^{6}$ W. S. Lockman, ${ }^{136}$ T. Loddenkoetter ${ }^{20}$ F. K. Loebinger, ${ }^{81}$ A. Loginov, ${ }^{175}$ C. W. Loh, ${ }^{167}$ T. Lohse, ${ }^{15}$ K. Lohwasser, ${ }^{47}$ M. Lokajicek, ${ }^{124}$ V. P. Lombardo,${ }^{4}$ R. E. Long, ${ }^{70}$ L. Lopes, ${ }^{123 a}$ D. Lopez Mateos, ${ }^{56}$ J. Lorenz, ${ }^{97}$ N. Lorenzo Martinez, ${ }^{114}$ M. Losada, ${ }^{161}$ P. Loscutoff, ${ }^{14}$ F. Lo Sterzo, ${ }^{131 a, 131 b}$ M. J. Losty, ${ }^{158 a, a}$ X. Lou, ${ }^{40}$ A. Lounis, ${ }^{114}$ K. F. Loureiro, ${ }^{161}$ J. Love, ${ }^{5}$ P. A. Love, ${ }^{70}$ A. J. Lowe, ${ }^{142, f}$ F. Lu, ${ }^{32 a}$ H. J. Lubatti, ${ }^{137}$ C. Luci, ${ }^{131 a, 131 b}$ A. Lucotte, ${ }^{54}$ A. Ludwig, ${ }^{43}$ D. Ludwig, ${ }^{41}$ I. Ludwig, ${ }^{47}$ J. Ludwig, ${ }^{47}$ F. Luehring,${ }^{59}$ G. Luijckx, ${ }^{104}$ W. Lukas, ${ }^{60}$ D. Lumb,${ }^{47}$ L. Luminari, ${ }^{131 a}$ E. Lund,${ }^{116}$ B. Lund-Jensen, ${ }^{146}$ B. Lundberg,${ }^{78}$ J. Lundberg,,${ }^{145 a, 145 b}$ O. Lundberg, ${ }^{145 a, 145 b}$ J. Lundquist, ${ }^{35}$ M. Lungwitz, ${ }^{80}$ D. Lynn, ${ }^{24}$ E. Lytken ${ }^{78}$ H. Ma,${ }^{24}$ L. L. Ma,${ }^{172}$ G. Maccarrone, ${ }^{46}$ A. Macchiolo, ${ }^{98}$ B. Maček, ${ }^{73}$ J. Machado Miguens, ${ }^{123 a}$ R. Mackeprang,${ }^{35}$ R. J. Madaras, ${ }^{14}$ H. J. Maddocks, ${ }^{70}$ W. F. Mader ${ }^{43}$ R. Maenner, ${ }^{57 c}$ T. Maeno, ${ }^{24}$ P. Mättig, ${ }^{174}$ S. Mättig, ${ }^{80}$ L. Magnoni,${ }^{162}$ E. Magradze,${ }^{53}$ K. Mahboubi ${ }^{47}$ S. Mahmoud ${ }^{72}$ G. Mahout, ${ }^{17}$ C. Maiani, ${ }^{135}$ C. Maidantchik,${ }^{23 a}$ A. Maio, ${ }^{123 a, c}$ S. Majewski, ${ }^{24}$ Y. Makida, ${ }^{64}$ N. Makovec, ${ }^{114}$ P. Mal,${ }^{135}$ B. Malaescu, ${ }^{29}$ Pa. Malecki, ${ }^{38}$ P. Malecki, ${ }^{38}$ V. P. Maleev, ${ }^{120}$ F. Malek,${ }^{54}$ U. Mallik,${ }^{61}$ D. Malon, ${ }^{5}$ C. Malone, ${ }^{142}$ S. Maltezos, ${ }^{9}$ V. Malyshev, ${ }^{106}$ S. Malyukov,${ }^{29}$ R. Mameghani, ${ }^{97}$ J. Mamuzic,${ }^{12 b}$ A. Manabe,${ }^{64}$ L. Mandelli, ${ }^{88 a}$ I. Mandić ${ }^{73}$ R. Mandrysch, ${ }^{15}$ J. Maneira, ${ }^{123 a}$ A. Manfredini,${ }^{98}$ P. S. Mangeard ${ }^{87}$ L. Manhaes de Andrade Filho, ${ }^{23 b}$ J. A. Manjarres Ramos, ${ }^{135}$ A. Mann, ${ }^{53}$ P. M. Manning, ${ }^{136}$

A. Manousakis-Katsikakis, ${ }^{8}$ B. Mansoulie, ${ }^{135}$ A. Mapelli, ${ }^{29}$ L. Mapelli, ${ }^{29}$ L. March,${ }^{79}$ J. F. Marchand ${ }^{28}$ F. Marchese, ${ }^{132 a, 132 b}$ G. Marchiori, ${ }^{77}$ M. Marcisovsky, ${ }^{124}$ C. P. Marino, ${ }^{168}$ F. Marroquim, ${ }^{23 a}$ Z. Marshall, ${ }^{29}$ F. K. Martens, ${ }^{157}$ L. F. Marti, ${ }^{16}$ S. Marti-Garcia, ${ }^{166}$ B. Martin, ${ }^{29}$ B. Martin, ${ }^{87}$ J. P. Martin, ${ }^{92}$ S. P. Martin, ${ }^{105}$ T. A. Martin, ${ }^{17}$ V. J. Martin, ${ }^{45}$ B. Martin dit Latour, ${ }^{48}$ S. Martin-Haugh, ${ }^{148}$ M. Martinez,${ }^{11}$ V. Martinez Outschoorn,${ }^{56}$ A. C. Martyniuk, ${ }^{168}$ M. Marx,${ }^{81}$ F. Marzano, ${ }^{131 a}$ A. Marzin, ${ }^{110}$ L. Masetti, ${ }^{80}$ T. Mashimo, ${ }^{154}$ R. Mashinistov, ${ }^{93}$ J. Masik, ${ }^{81}$ A. L. Maslennikov, ${ }^{106}$ I. Massa, ${ }^{19 a, 19 b}$ G. Massaro, ${ }^{104}$ N. Massol, ${ }^{4}$ P. Mastrandrea,${ }^{147}$ A. Mastroberardino, ${ }^{36 a, 36 b}$ T. Masubuchi, ${ }^{154}$ P. Matricon, ${ }^{114}$ H. Matsunaga, ${ }^{154}$ T. Matsushita, ${ }^{65}$ C. Mattravers, ${ }^{117, d}$ J. Maurer, ${ }^{82}$ S. J. Maxfield, ${ }^{72}$ A. Mayne, ${ }^{138}$ R. Mazini, ${ }^{150}$ M. Mazur ${ }^{20}$ L. Mazzaferro, ${ }^{132 a, 132 b}$ M. Mazzanti, ${ }^{88 a}$ J. Mc Donald, ${ }^{84}$ S. P. Mc Kee, ${ }^{86}$ A. McCarn, ${ }^{164}$ R. L. McCarthy, ${ }^{147}$ T. G. McCarthy, ${ }^{28}$ N. A. McCubbin, ${ }^{128}$ K. W. McFarlane, ${ }^{55, a}$ J. A. Mcfayden, ${ }^{138}$ G. Mchedlidze, ${ }^{50 b}$ T. Mclaughlan, ${ }^{17}$ S. J. McMahon, ${ }^{128}$ R. A. McPherson, ${ }^{168,1}$ A. Meade, ${ }^{83}$ J. Mechnich, ${ }^{104}$ M. Mechtel,${ }^{174}$ M. Medinnis, ${ }^{41}$ R. Meera-Lebbai, ${ }^{110}$ T. Meguro, ${ }^{115}$ R. Mehdiyev, ${ }^{92}$ S. Mehlhase, ${ }^{35}$ A. Mehta, ${ }^{72}$ K. Meier, ${ }^{57 a}$ B. Meirose,${ }^{78}$ C. Melachrinos, ${ }^{30}$ B. R. Mellado Garcia, ${ }^{172}$ F. Meloni, ${ }^{88 a, 88 b}$ L. Mendoza Navas, ${ }^{161}$ Z. Meng, ${ }^{150, v}$ A. Mengarelli, ${ }^{19 a, 19 b}$ S. Menke, ${ }^{98}$ E. Meoni, ${ }^{160}$ K. M. Mercurio, ${ }^{56}$ P. Mermod, ${ }^{48}$ L. Merola, ${ }^{101 \mathrm{a}, 101 \mathrm{~b}}$ C. Meroni, ${ }^{88 \mathrm{a}}$ F. S. Merritt, ${ }^{30}$ H. Merritt, ${ }^{108}$ A. Messina, ${ }^{29, z}$ J. Metcalfe, ${ }^{24}$ A. S. Mete, ${ }^{162}$ C. Meyer, ${ }^{80}$ C. Meyer, ${ }^{30}$ J-P. Meyer, ${ }^{135}$ J. Meyer, ${ }^{173}$ J. Meyer, ${ }^{53}$ T. C. Meyer, ${ }^{29}$ J. Miao, ${ }^{32 d}$ S. Michal,${ }^{29}$ L. Micu, ${ }^{25 a}$ R. P. Middleton, ${ }^{128}$ S. Migas, ${ }^{72}$ L. Mijović, ${ }^{135}$ G. Mikenberg, ${ }^{171}$ M. Mikestikova, ${ }^{124}$ M. Mikuž ${ }^{73}$ D. W. Miller, ${ }^{30}$ R. J. Miller, ${ }^{87}$ W. J. Mills, ${ }^{167}$ C. Mills, ${ }^{56}$ A. Milov, ${ }^{171}$ D. A. Milstead, ${ }^{145 a, 145 b}$ D. Milstein,${ }^{171}$ A. A. Minaenko, ${ }^{127}$ M. Miñano Moya ${ }^{166}$ I. A. Minashvili, ${ }^{63}$ A. I. Mincer,${ }^{107}$ B. Mindur, ${ }^{37}$ M. Mineev, ${ }^{63}$ Y. Ming, ${ }^{172}$ L. M. Mir, ${ }^{11}$ G. Mirabelli, ${ }^{131 a}$ J. Mitrevski, ${ }^{136}$ V. A. Mitsou, ${ }^{166}$ S. Mitsui, ${ }^{64}$ P. S. Miyagawa, ${ }^{138}$ J. U. Mjörnmark, ${ }^{78}$ T. Moa,${ }^{145 a, 145 b}$ V. Moeller, ${ }^{27}$ K. Mönig, ${ }^{41}$ N. Möser,${ }^{20}$ S. Mohapatra,${ }^{147}$ W. Mohr, ${ }^{47}$ R. Moles-Valls, ${ }^{166}$ J. Monk,${ }^{76}$ E. Monnier, ${ }^{82}$ J. Montejo Berlingen, ${ }^{11}$ F. Monticelli, ${ }^{69}$ S. Monzani, ${ }^{19 a, 19 b}$ R. W. Moore, ${ }^{2}$ G. F. Moorhead,${ }^{85}$ C. Mora Herrera, ${ }^{48}$ A. Moraes,${ }^{52}$ N. Morange, ${ }^{135}$ J. Morel,${ }^{53}$ G. Morello, ${ }^{36 a, 36 b}$ D. Moreno, ${ }^{80}$ M. Moreno Llácer, ${ }^{166}$ P. Morettini, ${ }^{49 a}$ M. Morgenstern, ${ }^{43}$ M. Morii, ${ }^{56}$ A. K. Morley, ${ }^{29}$ G. Mornacchi, ${ }^{29}$ J. D. Morris, ${ }^{74}$ L. Morvaj, ${ }^{100}$ H. G. Moser, ${ }^{98}$ M. Mosidze, ${ }^{50 b}$ J. Moss, ${ }^{108}$ R. Mount, ${ }^{142}$ E. Mountricha, ${ }^{9, a a}$ S. V. Mouraviev, ${ }^{93, a}$ E. J. W. Moyse ${ }^{83}$ F. Mueller, ${ }^{57 a}$ J. Mueller, ${ }^{122}$ K. Mueller, ${ }^{20}$ T. A. Müller, ${ }^{97}$ T. Mueller, ${ }^{80}$ D. Muenstermann, ${ }^{29}$ Y. Munwes, ${ }^{152}$ W. J. Murray,${ }^{128}$ I. Mussche, ${ }^{104}$ E. Musto, ${ }^{101 a, 101 b}$ A. G. Myagkov, ${ }^{127}$ M. Myska ${ }^{124}$ J. Nadal, ${ }^{11}$ K. Nagai,${ }^{159}$ R. Nagai, ${ }^{156}$ K. Nagano, ${ }^{64}$ A. Nagarkar, ${ }^{108}$ Y. Nagasaka, ${ }^{58}$ M. Nagel,${ }^{98}$ A. M. Nairz, ${ }^{29}$ Y. Nakahama, ${ }^{29}$ K. Nakamura, ${ }^{154}$ T. Nakamura, ${ }^{154}$ I. Nakano, ${ }^{109}$ G. Nanava, ${ }^{20}$ A. Napier,${ }^{160}$ R. Narayan, ${ }^{57 b}$ M. Nash, ${ }^{76, d}$ T. Nattermann, ${ }^{20}$ T. Naumann, ${ }^{41}$ G. Navarro, ${ }^{161}$ H. A. Neal, ${ }^{86}$ P. Yu. Nechaeva, ${ }^{93}$ T. J. Neep, ${ }^{81}$ A. Negri, ${ }^{118 \mathrm{a}, 118 \mathrm{~b}}$ G. Negri, ${ }^{29}$ M. Negrini, ${ }^{19 \mathrm{a}}$ S. Nektarijevic, ${ }^{48}$ A. Nelson, ${ }^{162}$ T. K. Nelson, ${ }^{142}$ S. Nemecek, ${ }^{124}$ P. Nemethy, ${ }^{107}$ A. A. Nepomuceno, ${ }^{23 a}$ M. Nessi, ${ }^{29, b b}$ M. S. Neubauer, ${ }^{164}$ M. Neumann,,${ }^{174}$ A. Neusiedl,${ }^{80}$ R. M. Neves, ${ }^{107}$ P. Nevski, ${ }^{24}$ P. R. Newman, ${ }^{17}$ V. Nguyen Thi Hong, ${ }^{135}$ R. B. Nickerson,,${ }^{117}$ R. Nicolaidou, ${ }^{135}$ B. Nicquevert, ${ }^{29}$ F. Niedercorn, ${ }^{114}$ J. Nielsen, ${ }^{136}$ N. Nikiforou, ${ }^{34}$ A. Nikiforov, ${ }^{15}$ V. Nikolaenko, ${ }^{127}$ I. Nikolic-Audit, ${ }^{77}$ K. Nikolics, ${ }^{48}$ K. Nikolopoulos,${ }^{17}$ H. Nilsen, ${ }^{47}$ P. Nilsson, ${ }^{7}$ Y. Ninomiya, ${ }^{154}$ A. Nisati, ${ }^{131 a}$ R. Nisius, ${ }^{98}$ T. Nobe,${ }^{156}$ L. Nodulman, ${ }^{5}$ M. Nomachi, ${ }^{115}$ I. Nomidis, ${ }^{153}$ S. Norberg, ${ }^{110}$ M. Nordberg, ${ }^{29}$ 
P. R. Norton, ${ }^{128}$ J. Novakova, ${ }^{125}$ M. Nozaki ${ }^{64}$ L. Nozka, ${ }^{112}$ I. M. Nugent, ${ }^{158 a}$ A.-E. Nuncio-Quiroz,${ }^{20}$ G. Nunes Hanninger, ${ }^{85}$ T. Nunnemann, ${ }^{97}$ E. Nurse, ${ }^{76}$ B. J. O’Brien, ${ }^{45}$ S. W. O'Neale, ${ }^{17, a}$ D. C. O'Neil,${ }^{141}$ V. O'Shea, ${ }^{52}$ L. B. Oakes, ${ }^{97}$ F. G. Oakham ${ }^{28, e}$ H. Oberlack, ${ }^{98}$ J. Ocariz ${ }^{77}$ A. Ochi,${ }^{65}$ S. Oda, ${ }^{68}$ S. Odaka, ${ }^{64}$ J. Odier,${ }^{82}$ H. Ogren, ${ }^{59}$ A. Oh, ${ }^{81}$ S. H. Oh, ${ }^{44}$ C. C. Ohm, ${ }^{29}$ T. Ohshima, ${ }^{100}$ H. Okawa,${ }^{24}$ Y. Okumura, ${ }^{30}$ T. Okuyama, ${ }^{154}$ A. Olariu, ${ }^{25 a}$ A. G. Olchevski, ${ }^{63}$ S. A. Olivares Pino, ${ }^{31 a}$ M. Oliveira, ${ }^{123 a, i}$ D. Oliveira Damazio, ${ }^{24}$ E. Oliver Garcia, ${ }^{166}$ D. Olivito, ${ }^{119}$ A. Olszewski, ${ }^{38}$ J. Olszowska, ${ }^{38}$ A. Onofre, ${ }^{123 a, c c}$ P. U. E. Onyisi, ${ }^{30}$ C. J. Oram, ${ }^{158 a}$ M. J. Oreglia, ${ }^{30}$ Y. Oren, ${ }^{152}$ D. Orestano, ${ }^{133 a, 133 b}$ N. Orlando, ${ }^{71 a, 71 b}$ I. Orlov, ${ }^{106}$ C. Oropeza Barrera, ${ }^{52}$ R. S. Orr, ${ }^{157}$ B. Osculati, ${ }^{49 a, 49 b}$ R. Ospanov, ${ }^{119}$ C. Osuna, ${ }^{11}$ G. Otero y Garzon, ${ }^{26}$ J. P. Ottersbach,,${ }^{104}$ M. Ouchrif, ${ }^{134 d}$ E. A. Ouellette, ${ }^{168}$ F. Ould-Saada, ${ }^{116}$ A. Ouraou, ${ }^{135}$ Q. Ouyang, ${ }^{32 a}$ A. Ovcharova, ${ }^{14}$ M. Owen, ${ }^{81}$ S. Owen, ${ }^{138}$ V. E. Ozcan, ${ }^{18 a}$ N. Ozturk, ${ }^{7}$

A. Pacheco Pages,${ }^{11}$ C. Padilla Aranda,${ }^{11}$ S. Pagan Griso, ${ }^{14}$ E. Paganis,${ }^{138}$ C. Pahl,${ }^{98}$ F. Paige,${ }^{24}$ P. Pais,${ }^{83}$ K. Pajchel, ${ }^{116}$ G. Palacino, ${ }^{158 b}$ C. P. Paleari, ${ }^{6}$ S. Palestini, ${ }^{29}$ D. Pallin ${ }^{33}$ A. Palma, ${ }^{123 a}$ J. D. Palmer, ${ }^{17}$ Y. B. Pan, ${ }^{172}$ E. Panagiotopoulou, ${ }^{9}$ P. Pani,${ }^{104}$ N. Panikashvili,${ }^{86}$ S. Panitkin, ${ }^{24}$ D. Pantea, ${ }^{25 a}$ A. Papadelis,${ }^{145 a}$

Th.D. Papadopoulou, ${ }^{9}$ A. Paramonov, ${ }^{5}$ D. Paredes Hernandez,${ }^{33}$ W. Park, ${ }^{24, d d}$ M. A. Parker, ${ }^{27}$ F. Parodi, ${ }^{49 a, 49 b}$ J. A. Parsons, ${ }^{34}$ U. Parzefall, ${ }^{47}$ S. Pashapour ${ }^{53}$ E. Pasqualucci, ${ }^{131 a}$ S. Passaggio, ${ }^{49 a}$ A. Passeri, ${ }^{133 a}$ F. Pastore, ${ }^{133 a, 133 b, a}$ Fr. Pastore,${ }^{75}$ G. Pásztor,${ }^{48, e e}$ S. Pataraia, ${ }^{174}$ N. Patel,${ }^{149}$ J. R. Pater, ${ }^{81}$ S. Patricelli, ${ }^{101 a, 101 b}$ T. Pauly, ${ }^{29}$ M. Pecsy, ${ }^{143 a}$ S. Pedraza Lopez, ${ }^{166}$ M. I. Pedraza Morales, ${ }^{172}$ S. V. Peleganchuk, ${ }^{106}$ D. Pelikan, ${ }^{165}$ H. Peng, ${ }^{32 b}$ B. Penning, ${ }^{30}$ A. Penson,${ }^{34}$ J. Penwell, ${ }^{59}$ M. Perantoni, ${ }^{23 a}$ K. Perez,${ }^{34, f f}$ T. Perez Cavalcanti, ${ }^{41}$ E. Perez Codina, ${ }^{158 a}$ M. T. Pérez García-Estañ, ${ }^{166}$ V. Perez Reale, ${ }^{34}$ L. Perini, ${ }^{88 a, 88 b}$ H. Pernegger, ${ }^{29}$ R. Perrino, ${ }^{71 a}$ P. Perrodo, ${ }^{4}$ V. D. Peshekhonov, ${ }^{63}$ K. Peters,${ }^{29}$ B. A. Petersen, ${ }^{29}$ J. Petersen, ${ }^{29}$ T. C. Petersen,${ }^{35}$ E. Petit, ${ }^{4}$ A. Petridis, ${ }^{153}$ C. Petridou, ${ }^{153}$ E. Petrolo, ${ }^{131 a}$ F. Petrucci, ${ }^{133 a, 133 b}$ D. Petschull,${ }^{41}$ M. Petteni, ${ }^{141}$ R. Pezoa,${ }^{31 b}$ A. Phan,${ }^{85}$ P. W. Phillips, ${ }^{128}$ G. Piacquadio,${ }^{29}$ A. Picazio,${ }^{48}$ E. Piccaro, ${ }^{74}$ M. Piccinini, ${ }^{19 a, 19 b}$ S. M. Piec,${ }^{41}$ R. Piegaia, ${ }^{26}$ D. T. Pignotti, ${ }^{108}$ J. E. Pilcher, ${ }^{30}$ A. D. Pilkington,${ }^{81}$ J. Pina, ${ }^{123 a, c}$ M. Pinamonti, ${ }^{163 a, 163 c}$ A. Pinder, ${ }^{117}$ J. L. Pinfold, ${ }^{2}$ B. Pinto, ${ }^{123 a}$ C. Pizio, ${ }^{88 a, 88 b}$ M. Plamondon, ${ }^{168}$ M.-A. Pleier, ${ }^{24}$ E. Plotnikova, ${ }^{63}$ A. Poblaguev, ${ }^{24}$ S. Poddar, ${ }^{57 a}$ F. Podlyski, ${ }^{33}$ L. Poggioli, ${ }^{114}$ D. Pohl,${ }^{20}$ M. Pohl,${ }^{48}$ G. Polesello, ${ }^{118 a}$ A. Policicchio, ${ }^{36 a, 36 b}$ A. Polini, ${ }^{19 a}$ J. Poll, ${ }^{74}$ V. Polychronakos, ${ }^{24}$ D. Pomeroy, ${ }^{22}$ K. Pommès, ${ }^{29}$ L. Pontecorvo, ${ }^{131 a}$ B. G. Pope,${ }^{87}$ G. A. Popeneciu, ${ }^{25 a}$ D. S. Popovic, ${ }^{12 \mathrm{a}}$ A. Poppleton, ${ }^{29}$ X. Portell Bueso, ${ }^{29}$ G. E. Pospelov, ${ }^{98}$ S. Pospisil, ${ }^{126}$ I. N. Potrap,${ }^{98}$ C. J. Potter, ${ }^{148}$ C. T. Potter, ${ }^{113}$ G. Poulard, ${ }^{29}$ J. Poveda,${ }^{59}$ V. Pozdnyakov, ${ }^{63}$ R. Prabhu, ${ }^{76}$ P. Pralavorio, ${ }^{82}$ A. Pranko, ${ }^{14}$ S. Prasad, ${ }^{29}$ R. Pravahan ${ }^{24}$ S. Prell,${ }^{62}$ K. Pretzl,${ }^{16}$ D. Price, ${ }^{59}$ J. Price,${ }^{72}$ L. E. Price,${ }^{5}$ D. Prieur, ${ }^{122}$ M. Primavera, ${ }^{71 a}$ K. Prokofiev, ${ }^{107}$ F. Prokoshin, ${ }^{31 b}$ S. Protopopescu, ${ }^{24}$ J. Proudfoot, ${ }^{5}$ X. Prudent, ${ }^{43}$ M. Przybycien, ${ }^{37}$ H. Przysiezniak, ${ }^{4}$ S. Psoroulas, ${ }^{20}$ E. Ptacek, ${ }^{113}$ E. Pueschel, ${ }^{83}$ J. Purdham,${ }^{86}$ M. Purohit,${ }^{24, d d}$ P. Puzo, ${ }^{114}$ Y. Pylypchenko, ${ }^{61}$ J. Qian, ${ }^{86}$ A. Quadt, ${ }^{53}$ D. R. Quarrie, ${ }^{14}$ W. B. Quayle, ${ }^{172}$ F. Quinonez, ${ }^{31 a}$ M. Raas, ${ }^{103}$ V. Radescu, ${ }^{41}$ P. Radloff, ${ }^{113}$ T. Rador, ${ }^{18 a}$ F. Ragusa, ${ }^{88 a, 88 b}$ G. Rahal, ${ }^{177}$ A. M. Rahimi, ${ }^{108}$ D. Rahm, ${ }^{24}$ S. Rajagopalan,${ }^{24}$ M. Rammensee,${ }^{47}$ M. Rammes, ${ }^{140}$ A. S. Randle-Conde ${ }^{39}$ K. Randrianarivony, ${ }^{28}$ F. Rauscher, ${ }^{97}$ T. C. Rave, ${ }^{47}$ M. Raymond, ${ }^{29}$ A. L. Read, ${ }^{116}$ D. M. Rebuzzi, ${ }^{118 a, 118 b}$ A. Redelbach, ${ }^{173}$ G. Redlinger, ${ }^{24}$ R. Reece, ${ }^{119}$ K. Reeves,${ }^{40}$ E. Reinherz-Aronis,${ }^{152}$ A. Reinsch, ${ }^{113}$ I. Reisinger, ${ }^{42}$ C. Rembser, ${ }^{29}$ Z. L. Ren, ${ }^{150}$ A. Renaud, ${ }^{114}$ M. Rescigno, ${ }^{131 a}$ S. Resconi, ${ }^{88 a}$ B. Resende, ${ }^{135}$ P. Reznicek, ${ }^{97}$ R. Rezvani, ${ }^{157}$ R. Richter, ${ }^{98}$ E. Richter-Was, ${ }^{4, \text { gg }}$ M. Ridel, ${ }^{77}$ M. Rijpstra, ${ }^{104}$ M. Rijssenbeek, ${ }^{147}$ A. Rimoldi, ${ }^{118 a, 118 b}$ L. Rinaldi, ${ }^{19 a}$ R. R. Rios,${ }^{39}$ I. Riu, ${ }^{11}$ G. Rivoltella, ${ }^{88 a, 88 b}$ F. Rizatdinova, ${ }^{111}$ E. Rizvi, ${ }^{74}$ S. H. Robertson, ${ }^{84,1}$ A. Robichaud-Veronneau, ${ }^{117}$ D. Robinson, ${ }^{27}$ J. E. M. Robinson ${ }^{81}$ A. Robson, ${ }^{52}$ J. G. Rocha de Lima, ${ }^{105}$ C. Roda ${ }^{121 a, 121 b}$ D. Roda Dos Santos, ${ }^{29}$ A. Roe,${ }^{53}$ S. Roe, ${ }^{29}$ O. Røhne, ${ }^{116}$ S. Rolli, ${ }^{160}$ A. Romaniouk, ${ }^{95}$ M. Romano, ${ }^{19 a, 19 b}$ G. Romeo, ${ }^{26}$ E. Romero Adam, ${ }^{166}$ N. Rompotis, ${ }^{137}$ L. Roos, ${ }^{77}$ E. Ros, ${ }^{166}$ S. Rosati, ${ }^{131 a}$ K. Rosbach, ${ }^{48}$ A. Rose,${ }^{148}$ M. Rose, ${ }^{75}$ G. A. Rosenbaum, ${ }^{157}$ E. I. Rosenberg, ${ }^{62}$ P. L. Rosendahl, ${ }^{13}$ O. Rosenthal, ${ }^{140}$ L. Rosselet, ${ }^{48}$ V. Rossetti, ${ }^{11}$ E. Rossi, ${ }^{131 \mathrm{a}, 131 \mathrm{~b}}$ L. P. Rossi ${ }^{49 a}$ M. Rotaru, ${ }^{25 \mathrm{a}}$ I. Roth, ${ }^{171}$ J. Rothberg, ${ }^{137}$ D. Rousseau, ${ }^{114}$ C. R. Royon, ${ }^{135}$ A. Rozanov, ${ }^{82}$ Y. Rozen, ${ }^{151}$ X. Ruan,,${ }^{32 a, h h}$ F. Rubbo,${ }^{11}$ I. Rubinskiy, ${ }^{41}$

N. Ruckstuhl, ${ }^{104}$ V. I. Rud,${ }^{96}$ C. Rudolph, ${ }^{43}$ G. Rudolph, ${ }^{60}$ F. Rühr, ${ }^{6}$ A. Ruiz-Martinez, ${ }^{62}$ L. Rumyantsev, ${ }^{63}$ Z. Rurikova, ${ }^{47}$ N. A. Rusakovich, ${ }^{63}$ J. P. Rutherfoord, ${ }^{6}$ C. Ruwiedel,${ }^{14, a}$ P. Ruzicka, ${ }^{124}$ Y. F. Ryabov, ${ }^{120}$ M. Rybar, ${ }^{125}$ G. Rybkin, ${ }^{114}$ N. C. Ryder, ${ }^{117}$ A. F. Saavedra, ${ }^{149}$ I. Sadeh,${ }^{152}$ H.F-W. Sadrozinski, ${ }^{136}$ R. Sadykov,${ }^{63}$

F. Safai Tehrani, ${ }^{131 a}$ H. Sakamoto, ${ }^{154}$ G. Salamanna, ${ }^{74}$ A. Salamon, ${ }^{132 a}$ M. Saleem, ${ }^{110}$ D. Salek, ${ }^{29}$ D. Salihagic, ${ }^{98}$ A. Salnikov ${ }^{142}$ J. Salt, ${ }^{166}$ B. M. Salvachua Ferrando, ${ }^{5}$ D. Salvatore,${ }^{36 a, 36 b}$ F. Salvatore, ${ }^{148}$ A. Salvucci, ${ }^{103}$ A. Salzburger ${ }^{29}$ D. Sampsonidis, ${ }^{153}$ B. H. Samset, ${ }^{116}$ A. Sanchez, ${ }^{101 a, 101 b}$ V. Sanchez Martinez, ${ }^{166}$ H. Sandaker, ${ }^{13}$ H. G. Sander, ${ }^{80}$ M. P. Sanders, ${ }^{97}$ M. Sandhoff, ${ }^{174}$ T. Sandoval, ${ }^{27}$ C. Sandoval, ${ }^{161}$ R. Sandstroem, ${ }^{98}$ D. P. C. Sankey, ${ }^{128}$

A. Sansoni,${ }^{46}$ C. Santamarina Rios, ${ }^{84}$ C. Santoni,${ }^{33}$ R. Santonico, ${ }^{132 a, 132 b}$ H. Santos, ${ }^{123 a}$ J. G. Saraiva, ${ }^{123 a}$ 
T. Sarangi, ${ }^{172}$ E. Sarkisyan-Grinbaum, ${ }^{7}$ F. Sarri, ${ }^{121 a, 121 b}$ G. Sartisohn, ${ }^{174}$ O. Sasaki, ${ }^{64}$ Y. Sasaki, ${ }^{154}$ N. Sasao,${ }^{66}$

I. Satsounkevitch, ${ }^{89}$ G. Sauvage, ${ }^{4, a}$ E. Sauvan, ${ }^{4}$ J. B. Sauvan, ${ }^{114}$ P. Savard, ${ }^{157, \text { e }}$ V. Savinov, ${ }^{122}$ D. O. Savu, ${ }^{29}$ L. Sawyer, ${ }^{24, \mathrm{n}}$ D. H. Saxon, ${ }^{52}$ J. Saxon, ${ }^{119}$ C. Sbarra, ${ }^{19 a}$ A. Sbrizzi, ${ }^{19 a, 19 b}$ D. A. Scannicchio, ${ }^{162}$ M. Scarcella, ${ }^{149}$ J. Schaarschmidt,${ }^{114}$ P. Schacht, ${ }^{98}$ D. Schaefer, ${ }^{119}$ U. Schäfer ${ }^{80}$ S. Schaepe,${ }^{20}$ S. Schaetzel,${ }^{57 b}$ A. C. Schaffer, ${ }^{114}$

D. Schaile, ${ }^{97}$ R. D. Schamberger, ${ }^{147}$ A. G. Schamov, ${ }^{106}$ V. Scharf, ${ }^{57 a}$ V. A. Schegelsky, ${ }^{120}$ D. Scheirich, ${ }^{86}$ M. Schernau, ${ }^{162}$ M. I. Scherzer, ${ }^{34}$ C. Schiavi, ${ }^{49 a, 49 b}$ J. Schieck, ${ }^{97}$ M. Schioppa,${ }^{36 a, 36 b}$ S. Schlenker, ${ }^{29}$ E. Schmidt, ${ }^{47}$ K. Schmieden, ${ }^{20}$ C. Schmitt, ${ }^{80}$ S. Schmitt, ${ }^{57 b}$ M. Schmitz, ${ }^{20}$ B. Schneider, ${ }^{16}$ U. Schnoor, ${ }^{43}$ A. Schoening, ${ }^{57 b}$ A. L. S. Schorlemmer, ${ }^{53}$ M. Schott, ${ }^{29}$ D. Schouten, ${ }^{158 a}$ J. Schovancova, ${ }^{124}$ M. Schram ${ }^{84}$ C. Schroeder, ${ }^{80}$ N. Schroer ${ }^{57 c}$ M. J. Schultens, ${ }^{20}$ J. Schultes, ${ }^{174}$ H.-C. Schultz-Coulon,${ }^{57 a}$ H. Schulz, ${ }^{15}$ M. Schumacher, ${ }^{47}$ B. A. Schumm, ${ }^{136} \mathrm{Ph}$. Schune, ${ }^{135}$ C. Schwanenberger, ${ }^{81}$ A. Schwartzman, ${ }^{142} \mathrm{Ph}$. Schwegler, ${ }^{98} \mathrm{Ph}$. Schwemling, ${ }^{77}$ R. Schwienhorst ${ }^{87}$ R. Schwierz, ${ }^{43}$ J. Schwindling, ${ }^{135}$ T. Schwindt, ${ }^{20}$ M. Schwoerer, ${ }^{4}$ G. Sciolla, ${ }^{22}$ W. G. Scott, ${ }^{128}$ J. Searcy, ${ }^{113}$ G. Sedov, ${ }^{41}$ E. Sedykh, ${ }^{120}$ S. C. Seidel, ${ }^{102}$ A. Seiden, ${ }^{136}$ F. Seifert, ${ }^{43}$ J. M. Seixas,${ }^{23 a}$ G. Sekhniaidze, ${ }^{101 a}$ S. J. Sekula, ${ }^{39}$ K. E. Selbach, ${ }^{45}$ D. M. Seliverstov, ${ }^{120}$ B. Sellden, ${ }^{145 a}$ G. Sellers, ${ }^{72}$ M. Seman, ${ }^{143 b}$ N. Semprini-Cesari, ${ }^{19 a, 19 b}$ C. Serfon, ${ }^{97}$ L. Serin, ${ }^{114}$ L. Serkin, ${ }^{53}$ R. Seuster, ${ }^{98}$ H. Severini, ${ }^{110}$ A. Sfyrla, ${ }^{29}$ E. Shabalina ${ }^{53}$ M. Shamim, ${ }^{113}$ L. Y. Shan, ${ }^{32 a}$ J. T. Shank, ${ }^{21}$ Q. T. Shao, ${ }^{85}$ M. Shapiro, ${ }^{14}$ P. B. Shatalov, ${ }^{94}$ K. Shaw, ${ }^{163 a, 163 c}$ D. Sherman, ${ }^{175}$ P. Sherwood, ${ }^{76}$ A. Shibata, ${ }^{107}$ S. Shimizu, ${ }^{100}$ M. Shimojima, ${ }^{99}$ T. Shin, ${ }^{55}$ M. Shiyakova, ${ }^{63}$ A. Shmeleva, ${ }^{93}$ M. J. Shochet,${ }^{30}$ D. Short,,${ }^{17}$ S. Shrestha,${ }^{62}$ E. Shulga,${ }^{95}$ M. A. Shupe,${ }^{6}$ P. Sicho, ${ }^{124}$ A. Sidoti, ${ }^{131 a}$ F. Siegert, ${ }^{47}$ Dj. Sijacki, ${ }^{12 a}$ O. Silbert, ${ }^{171}$ J. Silva, ${ }^{123 a}$ Y. Silver, ${ }^{152}$ D. Silverstein,,${ }^{142}$

S. B. Silverstein, ${ }^{145 a}$ V. Simak, ${ }^{126}$ O. Simard,${ }^{135}$ Lj. Simic, ${ }^{12 a}$ S. Simion,,${ }^{114}$ E. Simioni,${ }^{80}$ B. Simmons,${ }^{76}$ R. Simoniello, ${ }^{88 a}, 88 \mathrm{~b}$ M. Simonyan, ${ }^{35}$ P. Sinervo, ${ }^{157}$ N. B. Sinev, ${ }^{113}$ V. Sipica, ${ }^{140}$ G. Siragusa, ${ }^{173}$ A. Sircar, ${ }^{24}$ A. N. Sisakyan, ${ }^{63, a}$ S.Yu. Sivoklokov, ${ }^{96}$ J. Sjölin, ${ }^{145 a, 145 b}$ T. B. Sjursen, ${ }^{13}$ L. A. Skinnari, ${ }^{14}$ H. P. Skottowe,${ }^{56}$

K. Skovpen, ${ }^{106}$ P. Skubic, ${ }^{110}$ M. Slater, ${ }^{17}$ T. Slavicek, ${ }^{126}$ K. Sliwa, ${ }^{160}$ V. Smakhtin, ${ }^{171}$ B. H. Smart, ${ }^{45}$

S.Yu. Smirnov, ${ }^{95}$ Y. Smirnov, ${ }^{95}$ L. N. Smirnova, ${ }^{96}$ O. Smirnova, ${ }^{78}$ B. C. Smith,${ }^{56}$ D. Smith, ${ }^{142}$ K. M. Smith, ${ }^{52}$ M. Smizanska, ${ }^{70}$ K. Smolek, ${ }^{126}$ A. A. Snesarev,${ }^{93}$ S. W. Snow, ${ }^{81}$ J. Snow, ${ }^{110}$ S. Snyder, ${ }^{24}$ R. Sobie, ${ }^{168,1}$ J. Sodomka, ${ }^{126}$ A. Soffer, ${ }^{152}$ C. A. Solans, ${ }^{166}$ M. Solar, ${ }^{126}$ J. Solc, ${ }^{126}$ E. Yu. Soldatov, ${ }^{95}$ U. Soldevila, ${ }^{166}$

E. Solfaroli Camillocci, ${ }^{131 a, 131 b}$ A. A. Solodkov, ${ }^{127}$ O. V. Solovyanov, ${ }^{127}$ V. Solovyev, ${ }^{120}$ N. Soni, ${ }^{85}$ V. Sopko, ${ }^{126}$ B. Sopko, ${ }^{126}$ M. Sosebee, ${ }^{7}$ R. Soualah, ${ }^{163 a, 163 c}$ A. Soukharev, ${ }^{106}$ S. Spagnolo, ${ }^{71 a, 71 b}$ F. Spanò,${ }^{75}$ R. Spighi, ${ }^{19 a}$ G. Spigo, ${ }^{29}$ R. Spiwoks, ${ }^{29}$ M. Spousta, ${ }^{125, \text { ii }}$ T. Spreitzer, ${ }^{157}$ B. Spurlock, ${ }^{7}$ R. D. St. Denis, ${ }^{52}$ J. Stahlman, ${ }^{119}$

R. Stamen, ${ }^{57 a}$ E. Stanecka, ${ }^{38}$ R. W. Stanek, ${ }^{5}$ C. Stanescu, ${ }^{133 a}$ M. Stanescu-Bellu, ${ }^{41}$ S. Stapnes, ${ }^{116}$

E. A. Starchenko, ${ }^{127}$ J. Stark, ${ }^{54}$ P. Staroba,${ }^{124}$ P. Starovoitov, ${ }^{41}$ R. Staszewski, ${ }^{38}$ A. Staude, ${ }^{97}$ P. Stavina, ${ }^{143 a, a}$ G. Steele, ${ }^{52}$ P. Steinbach, ${ }^{43}$ P. Steinberg, ${ }^{24}$ I. Stekl, ${ }^{126}$ B. Stelzer, ${ }^{141}$ H. J. Stelzer,${ }^{87}$ O. Stelzer-Chilton, ${ }^{158 a}$ H. Stenzel, ${ }^{51}$ S. Stern, ${ }^{98}$ G. A. Stewart ${ }^{29}$ J. A. Stillings,${ }^{20}$ M. C. Stockton,${ }^{84}$ K. Stoerig, ${ }^{47}$ G. Stoicea, ${ }^{25 a}$ S. Stonjek, ${ }^{98}$ P. Strachota, ${ }^{125}$ A. R. Stradling, ${ }^{7}$ A. Straessner, ${ }^{43}$ J. Strandberg, ${ }^{146}$ S. Strandberg, ${ }^{145 a, 145 b}$ A. Strandlie, ${ }^{116}$ M. Strang, ${ }^{108}$ E. Strauss, ${ }^{142}$ M. Strauss, ${ }^{110}$ P. Strizenec, ${ }^{143 b}$ R. Ströhmer, ${ }^{173}$ D. M. Strom, ${ }^{113}$ J. A. Strong, ${ }^{75, a}$ R. Stroynowski, ${ }^{39}$ J. Strube, ${ }^{128}$ B. Stugu, ${ }^{13}$ I. Stumer, ${ }^{24, a}$ J. Stupak, ${ }^{147}$ P. Sturm, ${ }^{174}$ N. A. Styles, ${ }^{41}$ D. A. Soh, ${ }^{150, x}$ D. Su, ${ }^{142}$ HS. Subramania, ${ }^{2}$ A. Succurro, ${ }^{11}$ Y. Sugaya, ${ }^{115}$ C. Suhr, ${ }^{105}$ M. Suk ${ }^{125}$ V. V. Sulin, ${ }^{93}$ S. Sultansoy, ${ }^{3 d}$ T. Sumida, ${ }^{66}$ X. Sun,${ }^{54}$ J. E. Sundermann, ${ }^{47}$ K. Suruliz, ${ }^{138}$ G. Susinno,,${ }^{36 a, 36 b}$ M. R. Sutton, ${ }^{148}$ Y. Suzuki, ${ }^{64}$ Y. Suzuki, ${ }^{65}$ M. Svatos, ${ }^{124}$ S. Swedish, ${ }^{167}$ I. Sykora, ${ }^{143 a}$ T. Sykora, ${ }^{125}$ J. Sánchez, ${ }^{166}$ D. Ta, ${ }^{104}$ K. Tackmann, ${ }^{41}$ A. Taffard, ${ }^{162}$ R. Tafirout, ${ }^{158 a}$ N. Taiblum, ${ }^{152}$ Y. Takahashi, ${ }^{100}$ H. Takai, ${ }^{24}$ R. Takashima, ${ }^{67} \mathrm{H}$. Takeda, ${ }^{65}$ T. Takeshita, ${ }^{139}$ Y. Takubo, ${ }^{64}$ M. Talby, ${ }^{82}$ A. Talyshev, ${ }^{106, g}$ M. C. Tamsett,${ }^{24}$ J. Tanaka, ${ }^{154}$ R. Tanaka, ${ }^{114}$ S. Tanaka, ${ }^{130}$ S. Tanaka, ${ }^{64}$ A. J. Tanasijczuk, ${ }^{141} \mathrm{~K}$. Tani, ${ }^{65} \mathrm{~N}$. Tannoury, ${ }^{82} \mathrm{~S}$. Tapprogge,${ }^{80} \mathrm{D}$. Tardif,${ }^{157} \mathrm{~S}$. Tarem,${ }^{151} \mathrm{~F}$. Tarrade ${ }^{28}$

G. F. Tartarelli, ${ }^{88 a}$ P. Tas, ${ }^{125}$ M. Tasevsky, ${ }^{124}$ E. Tassi,${ }^{36 a, 36 b}$ M. Tatarkhanov,${ }^{14}$ Y. Tayalati, ${ }^{134 d}$ C. Taylor,${ }^{76}$ F. E. Taylor, ${ }^{91}$ G. N. Taylor ${ }^{85}$ W. Taylor, ${ }^{158 b}$ M. Teinturier,${ }^{114}$ F. A. Teischinger ${ }^{29}$ M. Teixeira Dias Castanheira, ${ }^{74}$ P. Teixeira-Dias, ${ }^{75}$ K. K. Temming,${ }^{47}$ H. Ten Kate, ${ }^{29}$ P. K. Teng, ${ }^{150}$ S. Terada ${ }^{64}$ K. Terashi, ${ }^{154}$ J. Terron, ${ }^{79}$ M. Testa, ${ }^{46}$

R. J. Teuscher, ${ }^{157,1}$ J. Therhaag, ${ }^{20}$ T. Theveneaux-Pelzer ${ }^{77}$ S. Thoma, ${ }^{47}$ J. P. Thomas, ${ }^{17}$ E. N. Thompson, ${ }^{34}$

P. D. Thompson, ${ }^{17}$ P. D. Thompson, ${ }^{157}$ A. S. Thompson, ${ }^{52}$ L. A. Thomsen, ${ }^{35}$ E. Thomson, ${ }^{119}$ M. Thomson, ${ }^{27}$ W. M. Thong ${ }^{85}$ R. P. Thun, ${ }^{86}$ F. Tian,${ }^{34}$ M. J. Tibbetts, ${ }^{14}$ T. Tic, ${ }^{124}$ V. O. Tikhomirov, ${ }^{93}$ Y. A. Tikhonov, ${ }^{106, g}$ S. Timoshenko, ${ }^{95}$ P. Tipton, ${ }^{175}$ S. Tisserant, ${ }^{82}$ T. Todorov, ${ }^{4}$ S. Todorova-Nova, ${ }^{160}$ B. Toggerson, ${ }^{162}$ J. Tojo, ${ }^{68}$ S. Tokár, ${ }^{143 a} \mathrm{~K}$. Tokushuku, ${ }^{64} \mathrm{~K}$. Tollefson, ${ }^{87} \mathrm{M}$. Tomoto, ${ }^{100} \mathrm{~L}$. Tompkins, ${ }^{30} \mathrm{~K}$. Toms,${ }^{102}$ A. Tonoyan, ${ }^{13} \mathrm{C}$. Topfel, ${ }^{16}$

N. D. Topilin, ${ }^{63}$ I. Torchiani, ${ }^{29}$ E. Torrence, ${ }^{113}$ H. Torres,${ }^{77}$ E. Torró Pastor, ${ }^{166}$ J. Toth, ${ }^{82, e e}$ F. Touchard, ${ }^{82}$ D. R. Tovey, ${ }^{138}$ T. Trefzger, ${ }^{173}$ L. Tremblet, ${ }^{29}$ A. Tricoli, ${ }^{29}$ I. M. Trigger, ${ }^{158 a}$ S. Trincaz-Duvoid,${ }^{77}$ M. F. Tripiana, ${ }^{69}$ 
N. Triplett, ${ }^{24}$ W. Trischuk, ${ }^{157}$ B. Trocmé,${ }^{54}$ C. Troncon, ${ }^{88 a}$ M. Trottier-McDonald,${ }^{141}$ M. Trzebinski, ${ }^{38}$ A. Trzupek, ${ }^{38}$ C. Tsarouchas, ${ }^{29}$ J.C-L. Tseng, ${ }^{117}$ M. Tsiakiris, ${ }^{104}$ P. V. Tsiareshka,${ }^{89}$ D. Tsionou, ${ }^{4, j j}$ G. Tsipolitis, ${ }^{9}$ S. Tsiskaridze,${ }^{11}$ V. Tsiskaridze, ${ }^{47}$ E. G. Tskhadadze, ${ }^{50 a}$ I. I. Tsukerman, ${ }^{94}$ V. Tsulaia, ${ }^{14}$ J.-W. Tsung, ${ }^{20}$ S. Tsuno, ${ }^{64}$ D. Tsybychev, ${ }^{147}$ A. Tua, ${ }^{138}$ A. Tudorache ${ }^{25 a}$ V. Tudorache, ${ }^{25 a}$ J. M. Tuggle,${ }^{30}$ M. Turala ${ }^{38}$ D. Turecek, ${ }^{126}$ I. Turk Cakir,${ }^{3 e}$ E. Turlay ${ }^{104}$ R. Turra, ${ }^{88 a, 88 b}$ P. M. Tuts, ${ }^{34}$ A. Tykhonov, ${ }^{73}$ M. Tylmad,,${ }^{145 a, 145 b}$ M. Tyndel, ${ }^{128}$ G. Tzanakos, ${ }^{8}$ K. Uchida, ${ }^{20}$ I. Ueda, ${ }^{154}$ R. Ueno, ${ }^{28}$ M. Ugland, ${ }^{13}$ M. Uhlenbrock,${ }^{20}$ M. Uhrmacher,${ }^{53}$ F. Ukegawa, ${ }^{159}$ G. Unal, ${ }^{29}$ A. Undrus, ${ }^{24}$ G. Unel,,${ }^{162}$ Y. Unno, ${ }^{64}$ D. Urbaniec, ${ }^{34}$ G. Usai, ${ }^{7}$ M. Uslenghi,,${ }^{18 a, 118 b}$ L. Vacavant, ${ }^{82}$ V. Vacek, ${ }^{126}$ B. Vachon, ${ }^{84}$ S. Vahsen, ${ }^{14}$ J. Valenta, ${ }^{124}$ S. Valentinetti, ${ }^{19 a, 19 b}$ A. Valero, ${ }^{166}$ S. Valkar, ${ }^{125}$ E. Valladolid Gallego, ${ }^{166}$ S. Vallecorsa, ${ }^{151}$ J. A. Valls Ferrer, ${ }^{166}$ P. C. Van Der Deijl, ${ }^{104}$ R. van der Geer, ${ }^{104}$ H. van der Graaf,,${ }^{104}$

R. Van Der Leeuw, ${ }^{104}$ E. van der Poel, ${ }^{104}$ D. van der Ster, ${ }^{29}$ N. van Eldik, ${ }^{29}$ P. van Gemmeren, ${ }^{5}$ I. van Vulpen, ${ }^{104}$ M. Vanadia, ${ }^{98}$ W. Vandelli, ${ }^{29}$ A. Vaniachine,${ }^{5}$ P. Vankov, ${ }^{41}$ F. Vannucci,${ }^{77}$ R. Vari, ${ }^{131 \text { a }}$ T. Varol,,${ }^{83}$ D. Varouchas, ${ }^{14}$ A. Vartapetian, ${ }^{7}$ K. E. Varvell, ${ }^{149}$ V. I. Vassilakopoulos, ${ }^{55}$ F. Vazeille, ${ }^{33}$ T. Vazquez Schroeder,${ }^{53}$ G. Vegni, ${ }^{88 a, 88 b}$ J. J. Veillet, ${ }^{114} \mathrm{~F}$. Veloso, ${ }^{123 a} \mathrm{R}$. Veness,${ }^{29} \mathrm{~S}$. Veneziano, ${ }^{131 \mathrm{a}} \mathrm{A}$. Ventura, ${ }^{71 \mathrm{a}, 71 \mathrm{~b}} \mathrm{D}$. Ventura ${ }^{83} \mathrm{M}$. Venturi, ${ }^{47}$ N. Venturi, ${ }^{157}$ V. Vercesi, ${ }^{118 a}$ M. Verducci, ${ }^{137}$ W. Verkerke, ${ }^{104}$ J. C. Vermeulen, ${ }^{104}$ A. Vest,${ }^{43}$ M. C. Vetterli, ${ }^{141, e}$ I. Vichou, ${ }^{164}$ T. Vickey, ${ }^{144 b, k k}$ O. E. Vickey Boeriu, ${ }^{144 b}$ G. H. A. Viehhauser, ${ }^{117}$ S. Viel, ${ }^{167}$ M. Villa, ${ }^{19 a, 19 b}$ M. Villaplana Perez, ${ }^{166}$ E. Vilucchi ${ }^{46}$ M. G. Vincter, ${ }^{28}$ E. Vinek, ${ }^{29}$ V. B. Vinogradov, ${ }^{63}$ M. Virchaux, ${ }^{135, a}$ J. Virzi, ${ }^{14}$ O. Vitells, ${ }^{171}$ M. Viti, ${ }^{41}$ I. Vivarelli, ${ }^{47}$ F. Vives Vaque, ${ }^{2}$ S. Vlachos, ${ }^{9}$ D. Vladoiu, ${ }^{97}$ M. Vlasak, ${ }^{126}$ A. Vogel,${ }^{20}$ P. Vokac, ${ }^{126}$ G. Volpi ${ }^{46}$ M. Volpi ${ }^{85}$ G. Volpini, ${ }^{88 a}$ H. von der Schmitt,${ }^{98}$ H. von Radziewski, ${ }^{47}$ E. von Toerne, ${ }^{20}$ V. Vorobel, ${ }^{125}$ V. Vorwerk, ${ }^{11}$ M. Vos, ${ }^{166}$ R. Voss, ${ }^{29}$ T. T. Voss, ${ }^{174}$ J. H. Vossebeld, ${ }^{72}$ N. Vranjes,,${ }^{135}$ M. Vranjes Milosavljevic, ${ }^{104}$ V. Vrba, ${ }^{124}$ M. Vreeswijk, ${ }^{104}$ T. Vu Anh,${ }^{47}$ R. Vuillermet,${ }^{29}$ I. Vukotic,${ }^{30}$ W. Wagner, ${ }^{174}$ P. Wagner, ${ }^{119} \mathrm{H}$. Wahlen, ${ }^{174} \mathrm{~S}$. Wahrmund, ${ }^{43}$ J. Wakabayashi, ${ }^{100} \mathrm{~S}$. Walch,${ }^{86} \mathrm{~J}$. Walder, ${ }^{70} \mathrm{R}$. Walker, ${ }^{97}$ W. Walkowiak, ${ }^{140}$ R. Wall,${ }^{175}$ P. Waller, ${ }^{72}$ B. Walsh, ${ }^{175}$ C. Wang, ${ }^{44}$ H. Wang, ${ }^{172}$ H. Wang, ${ }^{32 b, 11}$ J. Wang, ${ }^{150}$ J. Wang, ${ }^{54}$ R. Wang, ${ }^{102}$ S. M. Wang, ${ }^{150}$ T. Wang,${ }^{20}$ A. Warburton, ${ }^{84}$ C. P. Ward,${ }^{27}$ M. Warsinsky,${ }^{47}$ A. Washbrook,${ }^{45}$ C. Wasicki, ${ }^{41}$ I. Watanabe, ${ }^{65}$ P. M. Watkins,${ }^{17}$ A. T. Watson,,${ }^{17}$ I. J. Watson, ${ }^{149}$ M. F. Watson, ${ }^{17}$ G. Watts, ${ }^{137}$ S. Watts, ${ }^{81}$ A. T. Waugh, ${ }^{149}$ B. M. Waugh, ${ }^{76}$ M. S. Weber, ${ }^{16}$ P. Weber, ${ }^{53}$ A. R. Weidberg, ${ }^{117}$ P. Weigell, ${ }^{98}$ J. Weingarten, ${ }^{53}$

C. Weiser, ${ }^{47}$ H. Wellenstein, ${ }^{22}$ P. S. Wells, ${ }^{29}$ T. Wenaus,${ }^{24}$ D. Wendland,${ }^{15}$ Z. Weng, ${ }^{150, x}$ T. Wengler, ${ }^{29}$ S. Wenig, ${ }^{29}$ N. Wermes,${ }^{20} \mathrm{M}$. Werner ${ }^{47}$ P. Werner ${ }^{29} \mathrm{M}$. Werth, ${ }^{162} \mathrm{M}$. Wessels, ${ }^{57 \mathrm{a}} \mathrm{J}$. Wetter, ${ }^{160} \mathrm{C}$. Weydert, ${ }^{54} \mathrm{~K}$. Whalen, ${ }^{28}$

S. J. Wheeler-Ellis, ${ }^{162}$ A. White, ${ }^{7}$ M. J. White, ${ }^{85}$ S. White, ${ }^{121 a, 121 b}$ S. R. Whitehead ${ }^{117}$ D. Whiteson, ${ }^{162}$ D. Whittington, ${ }^{59}$ F. Wicek, ${ }^{114}$ D. Wicke, ${ }^{174}$ F. J. Wickens, ${ }^{128}$ W. Wiedenmann, ${ }^{172}$ M. Wielers, ${ }^{128}$ P. Wienemann, ${ }^{20}$ C. Wiglesworth ${ }^{74}$ L. A. M. Wiik-Fuchs, ${ }^{47}$ P. A. Wijeratne, ${ }^{76}$ A. Wildauer, ${ }^{98}$ M. A. Wildt,${ }^{41, t}$ I. Wilhelm, ${ }^{125}$ H. G. Wilkens, ${ }^{29}$ J.Z. Will, ${ }^{97}$ E. Williams,${ }^{34}$ H. H. Williams, ${ }^{119}$ W. Willis, ${ }^{34}$ S. Willocq,${ }^{83}$ J. A. Wilson, ${ }^{17}$ M. G. Wilson, ${ }^{142}$ A. Wilson, ${ }^{86}$ I. Wingerter-Seez, ${ }^{4}$ S. Winkelmann, ${ }^{47}$ F. Winklmeier, ${ }^{29}$ M. Wittgen, ${ }^{142}$ S. J. Wollstadt, ${ }^{80}$ M. W. Wolter, ${ }^{38}$ H. Wolters, ${ }^{123 a, i}$ W. C. Wong, ${ }^{40}$ G. Wooden, ${ }^{86}$ B. K. Wosiek, ${ }^{38}$ J. Wotschack, ${ }^{29}$ M. J. Woudstra, ${ }^{81}$ K. W. Wozniak, ${ }^{38}$ K. Wraight, ${ }^{52}$ M. Wright, ${ }^{52}$ B. Wrona, ${ }^{72}$ S. L. Wu, ${ }^{172}$ X. Wu ${ }^{48}$ Y. Wu, ${ }^{32 b, m m}$ E. Wulf, ${ }^{34}$ B. M. Wynne, ${ }^{45}$ S. Xella, ${ }^{35}$ M. Xiao, ${ }^{135}$ S. Xie, ${ }^{47}$ C. Xu, ${ }^{32 b \text {,aa }}$ D. Xu, ${ }^{138}$ B. Yabsley, ${ }^{149}$ S. Yacoob, ${ }^{144 a, n n}$ M. Yamada ${ }^{64}$ H. Yamaguchi, ${ }^{154}$ A. Yamamoto, ${ }^{64}$ K. Yamamoto, ${ }^{62}$ S. Yamamoto,${ }^{154}$ T. Yamamura, ${ }^{154}$ T. Yamanaka, ${ }^{154}$ J. Yamaoka, ${ }^{44}$ T. Yamazaki, ${ }^{154}$ Y. Yamazaki, ${ }^{65}$ Z. Yan, ${ }^{21}$ H. Yang, ${ }^{86}$ U. K. Yang, ${ }^{81}$ Y. Yang, ${ }^{59}$ Z. Yang, ${ }^{145 a, 145 b}$ S. Yanush, ${ }^{90}$ L. Yao, ${ }^{32 a}$ Y. Yao, ${ }^{14}$ Y. Yasu, ${ }^{64}$ G. V. Ybeles Smit, ${ }^{129}$ J. Ye, ${ }^{39}$ S. Ye, ${ }^{24}$ M. Yilmaz, ${ }^{3 c}$ R. Yoosoofmiya, ${ }^{122}$ K. Yorita, ${ }^{170}$ R. Yoshida, ${ }^{5}$ C. Young, ${ }^{142}$ C. J. Young, ${ }^{117}$ S. Youssef, ${ }^{21}$ D. Yu, ${ }^{24}$ J. Yu, ${ }^{7}$ J. Yu, ${ }^{111}$ L. Yuan, ${ }^{65}$ A. Yurkewicz, ${ }^{105}$ B. Zabinski, ${ }^{38}$ R. Zaidan, ${ }^{61}$ A. M. Zaitsev, ${ }^{127}$ Z. Zajacova, ${ }^{29}$ L. Zanello, ${ }^{131 a, 131 b}$ D. Zanzi, ${ }^{98}$ A. Zaytsev, ${ }^{24}$ C. Zeitnitz, ${ }^{174}$ M. Zeman, ${ }^{124}$ A. Zemla,${ }^{38}$ C. Zendler ${ }^{20}$ O. Zenin, ${ }^{127}$ T. Ženišs, ${ }^{143 a}$ Z. Zinonos, ${ }^{121 \mathrm{a}, 121 \mathrm{~b}}$ S. Zenz, ${ }^{14}$ D. Zerwas, ${ }^{114}$ G. Zevi della Porta, ${ }^{56}$ Z. Zhan, ${ }^{32 \mathrm{~d}}$ D. Zhang, ${ }^{32 \mathrm{~b}, 1 \mathrm{l}}$ H. Zhang, ${ }^{87} \mathrm{~J}$. Zhang, ${ }^{5}$ X. Zhang, ${ }^{32 \mathrm{~d}}$ Z. Zhang, ${ }^{114}$ L. Zhao ${ }^{107}$ T. Zhao, ${ }^{137}$ Z. Zhao, ${ }^{32 \mathrm{~b}}$ A. Zhemchugov, ${ }^{63} \mathrm{~J}$. Zhong, ${ }^{117}$ B. Zhou, ${ }^{86}$ N. Zhou, ${ }^{162}$ Y. Zhou, ${ }^{150}$ C. G. Zhu, ${ }^{32 d}$ H. Zhu, ${ }^{41}$ J. Zhu, ${ }^{86}$ Y. Zhu, ${ }^{32 b}$ X. Zhuang, ${ }^{97}$ V. Zhuravlov, ${ }^{98}$ D. Zieminska, ${ }^{59}$ N. I. Zimin, ${ }^{63}$ R. Zimmermann, ${ }^{20}$ S. Zimmermann, ${ }^{20}$ S. Zimmermann,${ }^{47}$ M. Ziolkowski, ${ }^{140}$ R. Zitoun, ${ }^{4}$ L. Živković, ${ }^{34}$ V. V. Zmouchko, ${ }^{127, a}$ G. Zobernig, ${ }^{172}$ A. Zoccoli, ${ }^{19 a, 19 b}$ M. zur Nedden, ${ }^{15}$ V. Zutshi, ${ }^{105}$ and L. Zwalinski ${ }^{29}$

\title{
(ATLAS Collaboration)
}

\author{
${ }^{1}$ Physics Department, SUNY Albany, Albany, New York, USA \\ ${ }^{2}$ Department of Physics, University of Alberta, Edmonton, Alberta, Canada \\ ${ }^{3 a}$ Department of Physics, Ankara University, Ankara, Turkey
}


${ }^{3 \mathrm{~b}}$ Department of Physics, Dumlupinar University, Kutahya, Turkey

${ }^{3 \mathrm{c}}$ Department of Physics, Gazi University, Ankara, Turkey

${ }^{3 \mathrm{~d}}$ Division of Physics, TOBB University of Economics and Technology, Ankara, Turkey

${ }^{3 \mathrm{e}}$ Turkish Atomic Energy Authority, Ankara, Turkey

${ }^{4} L A P P, C N R S / I N 2 P 3$ and Université de Savoie, Annecy-le-Vieux, France

${ }^{5}$ High Energy Physics Division, Argonne National Laboratory, Argonne, Illinois, USA

${ }^{6}$ Department of Physics, University of Arizona, Tucson, Arizona, USA

${ }^{7}$ Department of Physics, The University of Texas at Arlington, Arlington, Texas, USA

${ }^{8}$ Physics Department, University of Athens, Athens, Greece

${ }^{9}$ Physics Department, National Technical University of Athens, Zografou, Greece

${ }^{10}$ Institute of Physics, Azerbaijan Academy of Sciences, Baku, Azerbaijan

${ }^{11}$ Institut de Física d'Altes Energies and Departament de Física de la Universitat Autònoma de Barcelona and ICREA, Barcelona, Spain

${ }^{12 \mathrm{a}}$ Institute of Physics, University of Belgrade, Belgrade, Serbia

${ }^{12 \mathrm{~b}}$ Vinca Institute of Nuclear Sciences, University of Belgrade, Belgrade, Serbia

${ }^{13}$ Department for Physics and Technology, University of Bergen, Bergen, Norway

${ }^{14}$ Physics Division, Lawrence Berkeley National Laboratory and University of California, Berkeley, California, USA

${ }^{15}$ Department of Physics, Humboldt University, Berlin, Germany

${ }^{16}$ Albert Einstein Center for Fundamental Physics and Laboratory for High Energy Physics, University of Bern, Bern, Switzerland

${ }^{17}$ School of Physics and Astronomy, University of Birmingham, Birmingham, United Kingdom

${ }^{18 a}$ Department of Physics, Bogazici University, Istanbul, Turkey

${ }^{18 \mathrm{~b}}$ Division of Physics, Dogus University, Istanbul, Turkey

${ }^{18 \mathrm{c} D e p a r t m e n t ~ o f ~ P h y s i c s ~ E n g i n e e r i n g, ~ G a z i a n t e p ~ U n i v e r s i t y, ~ G a z i a n t e p, ~ T u r k e y ~}$

${ }^{18 \mathrm{~d}}$ Department of Physics, Istanbul Technical University, Istanbul, Turkey

${ }^{19 a}$ INFN Sezione di Bologna, Italy

${ }^{19 \mathrm{~b}}$ Dipartimento di Fisica, Università di Bologna, Bologna, Italy

${ }^{20}$ Physikalisches Institut, University of Bonn, Bonn, Germany

${ }^{21}$ Department of Physics, Boston University, Boston, Massachusetts, USA

${ }^{22}$ Department of Physics, Brandeis University, Waltham, Massachusetts, USA

${ }^{23 a}$ Universidade Federal do Rio De Janeiro COPPE/EE/IF, Rio de Janeiro, Brazil

${ }^{23 \mathrm{~b}}$ Federal University of Juiz de Fora (UFJF), Juiz de Fora, Brazil

${ }^{23 \mathrm{c}}$ Federal University of Sao Joao del Rei (UFSJ), Sao Joao del Rei, Brazil

${ }^{23 \mathrm{~d}}$ Instituto de Fisica, Universidade de Sao Paulo, Sao Paulo, Brazil

${ }^{24}$ Physics Department, Brookhaven National Laboratory, Upton, New York, USA

${ }^{25 \mathrm{a}}$ National Institute of Physics and Nuclear Engineering, Bucharest, Romania

${ }^{25 \mathrm{~b}}$ University Politehnica Bucharest, Bucharest, Romania

${ }^{25 c}$ West University in Timisoara, Timisoara, Romania

${ }^{26}$ Departamento de Física, Universidad de Buenos Aires, Buenos Aires, Argentina

${ }^{27}$ Cavendish Laboratory, University of Cambridge, Cambridge, United Kingdom

${ }^{28}$ Department of Physics, Carleton University, Ottawa, Ontario, Canada

${ }^{29}$ CERN, Geneva, Switzerland

${ }^{30}$ Enrico Fermi Institute, University of Chicago, Chicago, Illinois, USA

${ }^{31 a}$ Departamento de Física, Pontificia Universidad Católica de Chile, Santiago, Chile

${ }^{31 \mathrm{~b}}$ Departamento de Física, Universidad Técnica Federico Santa María, Valparaíso, Chile

${ }^{32 \mathrm{a}}$ Institute of High Energy Physics, Chinese Academy of Sciences, Beijing, China

${ }^{32 \mathrm{~b}}$ Department of Modern Physics, University of Science and Technology of China, Anhui, China

${ }^{32 \mathrm{c}}$ Department of Physics, Nanjing University, Jiangsu, China

${ }^{32 \mathrm{~d}}$ School of Physics, Shandong University, Shandong, China

${ }^{33}$ Laboratoire de Physique Corpusculaire, Clermont Université and Université Blaise Pascal and CNRS/IN2P3,

Aubiere Cedex, France

${ }^{34}$ Nevis Laboratory, Columbia University, Irvington, New York, USA

${ }^{35}$ Niels Bohr Institute, University of Copenhagen, Kobenhavn, Denmark

${ }^{36 a}$ INFN Gruppo Collegato di Cosenza, Italy

${ }^{36 \mathrm{~b}}$ Dipartimento di Fisica, Università della Calabria, Arcavata di Rende, Italy

${ }^{37}$ AGH University of Science and Technology, Faculty of Physics and Applied Computer Science, Krakow, Poland

${ }^{38}$ The Henryk Niewodniczanski Institute of Nuclear Physics, Polish Academy of Sciences, Krakow, Poland

${ }^{39}$ Physics Department, Southern Methodist University, Dallas, Texas, USA

${ }^{40}$ Physics Department, University of Texas at Dallas, Richardson, Texas, USA

${ }^{41}$ DESY, Hamburg and Zeuthen, Germany

${ }^{42}$ Institut für Experimentelle Physik IV, Technische Universität Dortmund, Dortmund, Germany

${ }^{43}$ Institut für Kern- und Teilchenphysik, Technical University Dresden, Dresden, Germany 
${ }^{44}$ Department of Physics, Duke University, Durham, North Carolina, USA

${ }^{45}$ SUPA - School of Physics and Astronomy, University of Edinburgh, Edinburgh, United Kingdom

${ }^{46}$ INFN Laboratori Nazionali di Frascati, Frascati, Italy

${ }^{47}$ Fakultät für Mathematik und Physik, Albert-Ludwigs-Universität, Freiburg, Germany

${ }^{48}$ Section de Physique, Université de Genève, Geneva, Switzerland

${ }^{49}$ INFN Sezione di Genova, Italy

${ }^{49 \mathrm{~b}}$ Dipartimento di Fisica, Università di Genova, Genova, Italy

${ }^{50 a}$ E. Andronikashvili Institute of Physics, Tbilisi State University, Tbilisi, Georgia

${ }^{50 \mathrm{~b}}$ High Energy Physics Institute, Tbilisi State University, Tbilisi, Georgia

${ }^{51}$ II Physikalisches Institut, Justus-Liebig-Universität Giessen, Giessen, Germany

${ }^{52}$ SUPA - School of Physics and Astronomy, University of Glasgow, Glasgow, United Kingdom

${ }^{53}$ II Physikalisches Institut, Georg-August-Universität, Göttingen, Germany

${ }^{54}$ Laboratoire de Physique Subatomique et de Cosmologie, Université Joseph Fourier and CNRS/IN2P3 and Institut National Polytechnique de Grenoble, Grenoble, France

${ }^{55}$ Department of Physics, Hampton University, Hampton, Virginia, USA

${ }^{56}$ Laboratory for Particle Physics and Cosmology, Harvard University, Cambridge, Massachusetts, USA

${ }^{57 a}$ Kirchhoff-Institut für Physik, Ruprecht-Karls-Universität Heidelberg, Heidelberg, Germany

${ }^{57 b}$ Physikalisches Institut, Ruprecht-Karls-Universität Heidelberg, Heidelberg, Germany

${ }^{57 c}$ ZITI Institut für technische Informatik, Ruprecht-Karls-Universität Heidelberg, Mannheim, Germany

${ }^{58}$ Faculty of Applied Information Science, Hiroshima Institute of Technology, Hiroshima, Japan

${ }^{59}$ Department of Physics, Indiana University, Bloomington, Indiana, USA

${ }^{60}$ Institut für Astro- und Teilchenphysik, Leopold-Franzens-Universität, Innsbruck, Austria

${ }^{61}$ University of Iowa, Iowa City, Iowa, USA

${ }^{62}$ Department of Physics and Astronomy, Iowa State University, Ames, Iowa, USA

${ }^{63}$ Joint Institute for Nuclear Research, JINR Dubna, Dubna, Russia

${ }^{64}$ KEK, High Energy Accelerator Research Organization, Tsukuba, Japan

${ }^{65}$ Graduate School of Science, Kobe University, Kobe, Japan

${ }^{66}$ Faculty of Science, Kyoto University, Kyoto, Japan

${ }^{67}$ Kyoto University of Education, Kyoto, Japan

${ }^{68}$ Department of Physics, Kyushu University, Fukuoka, Japan

${ }^{69}$ Instituto de Física La Plata, Universidad Nacional de La Plata and CONICET, La Plata, Argentina

${ }^{70}$ Physics Department, Lancaster University, Lancaster, United Kingdom

${ }^{71 \mathrm{a}}$ INFN Sezione di Lecce, Italy

${ }^{71 \mathrm{~b}}$ Dipartimento di Matematica e Fisica, Università del Salento, Lecce, Italy

${ }^{72}$ Oliver Lodge Laboratory, University of Liverpool, Liverpool, United Kingdom

${ }^{73}$ Department of Physics, Jožef Stefan Institute and University of Ljubljana, Ljubljana, Slovenia

${ }^{74}$ School of Physics and Astronomy, Queen Mary University of London, London, United Kingdom

${ }^{75}$ Department of Physics, Royal Holloway University of London, Surrey, United Kingdom

${ }^{76}$ Department of Physics and Astronomy, University College London, London, United Kingdom

${ }^{77}$ Laboratoire de Physique Nucléaire et de Hautes Energies, UPMC and Université Paris-Diderot and CNRS/IN2P3, Paris, France

${ }^{78}$ Fysiska institutionen, Lunds universitet, Lund, Sweden

${ }^{79}$ Departamento de Fisica Teorica C-15, Universidad Autonoma de Madrid, Madrid, Spain

${ }^{80}$ Institut für Physik, Universität Mainz, Mainz, Germany

${ }^{81}$ School of Physics and Astronomy, University of Manchester, Manchester, United Kingdom

${ }^{82}$ CPPM, Aix-Marseille Université and CNRS/IN2P3, Marseille, France

${ }^{83}$ Department of Physics, University of Massachusetts, Amherst, Massachusetts, USA

${ }^{84}$ Department of Physics, McGill University, Montreal, Quebec, Canada

${ }^{85}$ School of Physics, University of Melbourne, Victoria, Australia

${ }^{86}$ Department of Physics, The University of Michigan, Ann Arbor, Michigan, USA

${ }^{87}$ Department of Physics and Astronomy, Michigan State University, East Lansing, Michigan, USA

${ }^{88}$ INFN Sezione di Milano, Italy

${ }^{88 \mathrm{~b}}$ Dipartimento di Fisica, Università di Milano, Milano, Italy

${ }^{89}$ B. I. Stepanov Institute of Physics, National Academy of Sciences of Belarus, Minsk, Republic of Belarus

${ }^{90}$ National Scientific and Educational Centre for Particle and High Energy Physics, Minsk, Republic of Belarus

${ }^{91}$ Department of Physics, Massachusetts Institute of Technology, Cambridge, Massachusetts, USA

${ }^{92}$ Group of Particle Physics, University of Montreal, Montreal, Quebec, Canada

${ }^{93}$ P. N. Lebedev Institute of Physics, Academy of Sciences, Moscow, Russia

${ }^{94}$ Institute for Theoretical and Experimental Physics (ITEP), Moscow, Russia

${ }^{95}$ Moscow Engineering and Physics Institute (MEPhI), Moscow, Russia

${ }^{96}$ Skobeltsyn Institute of Nuclear Physics, Lomonosov Moscow State University, Moscow, Russia

${ }^{97}$ Fakultät für Physik, Ludwig-Maximilians-Universität München, München, Germany 
${ }^{98}$ Max-Planck-Institut für Physik (Werner-Heisenberg-Institut), München, Germany

${ }^{99}$ Nagasaki Institute of Applied Science, Nagasaki, Japan

${ }^{100}$ Graduate School of Science and Kobayashi-Maskawa Institute, Nagoya University, Nagoya, Japan

${ }^{101}$ INFN Sezione di Napoli, Italy

${ }^{101 \mathrm{~b}}$ Dipartimento di Scienze Fisiche, Università di Napoli, Napoli, Italy

${ }^{102}$ Department of Physics and Astronomy, University of New Mexico, Albuquerque, New Mexico, USA

${ }^{103}$ Institute for Mathematics, Astrophysics and Particle Physics, Radboud University Nijmegen/Nikhef, Nijmegen, Netherlands

${ }^{104}$ Nikhef National Institute for Subatomic Physics and University of Amsterdam, Amsterdam, Netherlands

${ }^{105}$ Department of Physics, Northern Illinois University, DeKalb, Illinois, USA

${ }^{106}$ Budker Institute of Nuclear Physics, SB RAS, Novosibirsk, Russia

${ }^{107}$ Department of Physics, New York University, New York, New York, USA

${ }^{108}$ Ohio State University, Columbus, Ohio, USA

${ }^{109}$ Faculty of Science, Okayama University, Okayama, Japan

${ }^{110}$ Homer L. Dodge Department of Physics and Astronomy, University of Oklahoma, Norman, Oklahoma, USA

${ }^{111}$ Department of Physics, Oklahoma State University, Stillwater, Oklahoma, USA

${ }^{112}$ Palacký University, RCPTM, Olomouc, Czech Republic

${ }^{113}$ Center for High Energy Physics, University of Oregon, Eugene, Oregon, USA

${ }^{114}$ LAL, Université Paris-Sud and CNRS/IN2P3, Orsay, France

${ }^{115}$ Graduate School of Science, Osaka University, Osaka, Japan

${ }^{116}$ Department of Physics, University of Oslo, Oslo, Norway

${ }^{117}$ Department of Physics, Oxford University, Oxford, United Kingdom

${ }^{118 \mathrm{a}}$ INFN Sezione di Pavia, Italy

${ }^{118 \mathrm{~b}}$ Dipartimento di Fisica, Università di Pavia, Pavia, Italy

${ }^{119}$ Department of Physics, University of Pennsylvania, Philadelphia, Pennsylvania, USA

${ }^{120}$ Petersburg Nuclear Physics Institute, Gatchina, Russia

${ }^{121 \mathrm{a}}$ INFN Sezione di Pisa, Italy

${ }^{121 \mathrm{~b}}$ Dipartimento di Fisica E. Fermi, Università di Pisa, Pisa, Italy

${ }^{122}$ Department of Physics and Astronomy, University of Pittsburgh, Pittsburgh, Pennsylvania, USA

${ }^{123 a}$ Laboratorio de Instrumentacao e Fisica Experimental de Particulas - LIP, Lisboa, Portugal

${ }^{123 \mathrm{~b}}$ Departamento de Fisica Teorica y del Cosmos and CAFPE, Universidad de Granada, Granada, Spain

${ }^{124}$ Institute of Physics, Academy of Sciences of the Czech Republic, Praha, Czech Republic

${ }^{125}$ Faculty of Mathematics and Physics, Charles University in Prague, Praha, Czech Republic

${ }^{126}$ Czech Technical University in Prague, Praha, Czech Republic

${ }^{127}$ State Research Center Institute for High Energy Physics, Protvino, Russia

${ }^{128}$ Particle Physics Department, Rutherford Appleton Laboratory, Didcot, United Kingdom

${ }^{129}$ Physics Department, University of Regina, Regina, Saskatchewan, Canada

${ }^{130}$ Ritsumeikan University, Kusatsu, Shiga, Japan

${ }^{131}$ INFN Sezione di Roma I, Italy

${ }^{131 \mathrm{~b}}$ Dipartimento di Fisica, Università La Sapienza, Roma, Italy

${ }^{132 a}$ INFN Sezione di Roma Tor Vergata, Italy

${ }^{132 \mathrm{~b}}$ Dipartimento di Fisica, Università di Roma Tor Vergata, Roma, Italy

${ }^{133 a}$ INFN Sezione di Roma Tre, Italy

${ }^{133 b}$ Dipartimento di Fisica, Università Roma Tre, Roma, Italy

${ }^{134 a}$ Faculté des Sciences Ain Chock, Réseau Universitaire de Physique des Hautes Energies - Université Hassan II, Casablanca, Morocco

${ }^{134 \mathrm{~b}}$ Centre National de l'Energie des Sciences Techniques Nucleaires, Rabat, Morocco

${ }^{134 \mathrm{c}}$ Faculté des Sciences Semlalia, Université Cadi Ayyad, LPHEA-Marrakech, Morocco

${ }^{134 \mathrm{~d}}$ Faculté des Sciences, Université Mohamed Premier and LPTPM, Oujda, Morocco

${ }^{134 \mathrm{e}}$ Faculté des sciences, Université Mohammed V-Agdal, Rabat, Morocco

${ }^{135}$ DSM/IRFU (Institut de Recherches sur les Lois Fondamentales de l'Univers), CEA Saclay (Commissariat a l'Energie Atomique), Gif-sur-Yvette, France

${ }^{136}$ Santa Cruz, Institute for Particle Physics, University of California Santa Cruz, Santa Cruz, California, USA

${ }^{137}$ Department of Physics, University of Washington, Seattle, Washington, USA

${ }^{138}$ Department of Physics and Astronomy, University of Sheffield, Sheffield, United Kingdom

${ }^{139}$ Department of Physics, Shinshu University, Nagano, Japan

${ }^{140}$ Fachbereich Physik, Universität Siegen, Siegen, Germany

${ }^{141}$ Department of Physics, Simon Fraser University, Burnaby, British Columbia, Canada

${ }^{142}$ SLAC National Accelerator Laboratory, Stanford, California, USA

${ }^{143 a}$ Faculty of Mathematics, Physics and Informatics, Comenius University, Bratislava, Slovak Republic

${ }^{143 b}$ Department of Subnuclear Physics, Institute of Experimental Physics of the Slovak Academy of Sciences, Kosice, Slovak Republic

${ }^{144 a}$ Department of Physics, University of Johannesburg, Johannesburg, South Africa 
${ }^{144 \mathrm{~b}}$ School of Physics, University of the Witwatersrand, Johannesburg, South Africa ${ }^{145 a}$ Department of Physics, Stockholm University, Sweden

${ }^{145 \mathrm{~b}}$ The Oskar Klein Center, Stockholm, Sweden

${ }^{146}$ Physics Department, Royal Institute of Technology, Stockholm, Sweden

${ }^{147}$ Departments of Physics and Astronomy and Chemistry, Stony Brook University, Stony Brook, New York, USA

${ }^{148}$ Department of Physics and Astronomy, University of Sussex, Brighton, United Kingdom

${ }^{149}$ School of Physics, University of Sydney, Sydney, Australia

${ }^{150}$ Institute of Physics, Academia Sinica, Taipei, Taiwan

${ }^{151}$ Department of Physics, Technion: Israel Institute of Technology, Haifa, Israel

${ }^{152}$ Raymond and Beverly Sackler School of Physics and Astronomy, Tel Aviv University, Tel Aviv, Israel

${ }^{153}$ Department of Physics, Aristotle University of Thessaloniki, Thessaloniki, Greece

${ }^{154}$ International Center for Elementary Particle Physics and Department of Physics, The University of Tokyo, Tokyo, Japan

${ }^{155}$ Graduate School of Science and Technology, Tokyo Metropolitan University, Tokyo, Japan

${ }^{156}$ Department of Physics, Tokyo Institute of Technology, Tokyo, Japan

${ }^{157}$ Department of Physics, University of Toronto, Toronto, Ontario, Canada

${ }^{158}$ TRIUMF, Vancouver, British Columbia, Canada

${ }^{158 b}$ Department of Physics and Astronomy, York University, Toronto, Ontario, Canada

${ }^{159}$ Institute of Pure and Applied Sciences, University of Tsukuba, 1-1-1 Tennodai, Tsukuba, Ibaraki 305-8571, Japan

${ }^{160}$ Science and Technology Center, Tufts University, Medford, Massachusetts, USA

${ }^{161}$ Centro de Investigaciones, Universidad Antonio Narino, Bogota, Colombia

${ }^{162}$ Department of Physics and Astronomy, University of California Irvine, Irvine, California, USA

${ }^{163 a}$ INFN Gruppo Collegato di Udine, Italy

${ }^{163 \mathrm{~b}}$ ICTP, Trieste, Italy

${ }^{163 c}$ Dipartimento di Chimica, Fisica e Ambiente, Università di Udine, Udine, Italy

${ }^{164}$ Department of Physics, University of Illinois, Urbana, Illinois, USA

${ }^{165}$ Department of Physics and Astronomy, University of Uppsala, Uppsala, Sweden

${ }^{166}$ Instituto de Física Corpuscular (IFIC) and Departamento de Física Atómica, Molecular y Nuclear and Departamento de Ingeniería Electrónica and Instituto de Microelectrónica de Barcelona (IMB-CNM), University of Valencia and CSIC, Valencia, Spain

${ }^{167}$ Department of Physics, University of British Columbia, Vancouver, British Columbia, Canada

${ }^{168}$ Department of Physics and Astronomy, University of Victoria, Victoria, British Columbia, Canada

${ }^{169}$ Department of Physics, University of Warwick, Coventry, United Kingdom

${ }^{170}$ Waseda University, Tokyo, Japan

${ }^{171}$ Department of Particle Physics, The Weizmann Institute of Science, Rehovot, Israel

${ }^{172}$ Department of Physics, University of Wisconsin, Madison, Wisconsin, USA

${ }^{173}$ Fakultät für Physik und Astronomie, Julius-Maximilians-Universität, Würzburg, Germany

${ }^{174}$ Fachbereich C Physik, Bergische Universität Wuppertal, Wuppertal, Germany

${ }^{175}$ Department of Physics, Yale University, New Haven, Connecticut, USA

${ }^{176}$ Yerevan Physics Institute, Yerevan, Armenia

${ }^{177}$ Domaine scientifique de la Doua, Centre de Calcul CNRS/IN2P3, Villeurbanne Cedex, France

${ }^{\mathrm{a}}$ Deceased.

${ }^{\mathrm{b}}$ Also at Laboratorio de Instrumentacao e Fisica Experimental de Particulas - LIP, Lisboa, Portugal.

${ }^{\mathrm{c}}$ Also at Faculdade de Ciencias and CFNUL, Universidade de Lisboa, Lisboa, Portugal.

${ }^{\mathrm{d}}$ Also at Particle Physics Department, Rutherford Appleton Laboratory, Didcot, United Kingdom.

${ }^{\mathrm{e}}$ Also at TRIUMF, Vancouver, British Columbia, Canada.

${ }^{\mathrm{f}}$ Also at Department of Physics, California State University, Fresno, CA, USA.

${ }^{\mathrm{g}}$ Also at Novosibirsk State University, Novosibirsk, Russia.

${ }^{\mathrm{h}}$ Also at Fermilab, Batavia, IL, USA.

${ }^{\mathrm{i}}$ Also at Department of Physics, University of Coimbra, Coimbra, Portugal.

${ }^{\mathrm{j}}$ Also at Department of Physics, UASLP, San Luis Potosi, Mexico.

${ }^{\mathrm{k}}$ Also at Università di Napoli Parthenope, Napoli, Italy.

${ }^{1}$ Also at Institute of Particle Physics (IPP), Canada.

${ }^{\mathrm{m}}$ Also at Department of Physics, Middle East Technical University, Ankara, Turkey.

${ }^{\mathrm{n}}$ Also at Louisiana Tech University, Ruston, LA, USA.

${ }^{\circ}$ Also at Dep Fisica and CEFITEC of Faculdade de Ciencias e Tecnologia, Universidade Nova de Lisboa, Caparica, Portugal.

${ }^{\mathrm{p}}$ Also at Department of Physics and Astronomy, University College London, London, United Kingdom.

${ }^{\mathrm{q}}$ Also at Group of Particle Physics, University of Montreal, Montreal, Quebec, Canada.

${ }^{\mathrm{r}}$ Also at Department of Physics, University of Cape Town, Cape Town, South Africa.

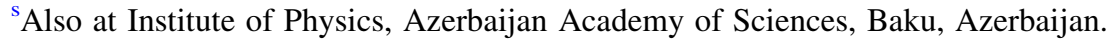


${ }^{\mathrm{t}}$ Also at Institut für Experimentalphysik, Universität Hamburg, Hamburg, Germany.

${ }^{\mathrm{u} A l s o}$ at Manhattan College, New York, NY, USA.

${ }^{v}$ Also at School of Physics, Shandong University, Shandong, China.

${ }^{w}$ Also at CPPM, Aix-Marseille Université and CNRS/IN2P3, Marseille, France.

${ }^{\mathrm{x}}$ Also at School of Physics and Engineering, Sun Yat-sen University, Guanzhou, China.

${ }^{y}$ Also at Academia Sinica Grid Computing, Institute of Physics, Academia Sinica, Taipei, Taiwan.

${ }^{\mathrm{z}}$ Also at Dipartimento di Fisica, Università La Sapienza, Roma, Italy.

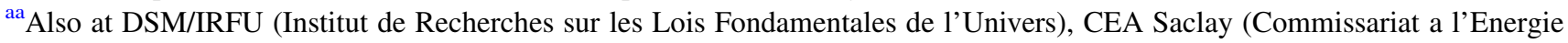
Atomique), Gif-sur-Yvette, France.

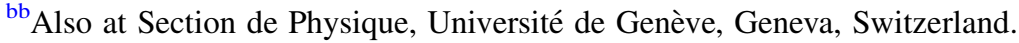

${ }^{\mathrm{cc}}$ Also at Departamento de Fisica, Universidade de Minho, Braga, Portugal.

${ }^{\mathrm{dd}}$ Also at Department of Physics and Astronomy, University of South Carolina, Columbia, SC, USA.

ee Also at Institute for Particle and Nuclear Physics, Wigner Research Centre for Physics, Budapest, Hungary.

${ }^{\mathrm{ff}}$ Also at California Institute of Technology, Pasadena, CA, USA.

${ }^{g g}$ Also at Institute of Physics, Jagiellonian University, Krakow, Poland.

${ }^{\text {hh }}$ Also at LAL, Université Paris-Sud and CNRS/IN2P3, Orsay, France.

${ }^{\text {ii }}$ Also at Nevis Laboratory, Columbia University, Irvington, NY, USA.

${ }^{\mathrm{jj}}$ Also at Department of Physics and Astronomy, University of Sheffield, Sheffield, United Kingdom.

${ }^{\mathrm{kk}}$ Also at Department of Physics, Oxford University, Oxford, United Kingdom.

${ }^{11}$ Also at Institute of Physics, Academia Sinica, Taipei, Taiwan

${ }^{\mathrm{mm}}$ Also at Department of Physics, The University of Michigan, Ann Arbor, MI, USA.

${ }^{n n}$ Also at Discipline of Physics, University of KwaZulu-Natal, Durban, South Africa. 